\title{
Automorphic Green Functions Associated with the Secondary Spherical Functions
}

\author{
Dedicated to Professor Yasutaka Ihara \\ By \\ Takayuki ODA* and Masao TsuzukI**
}

\section{$\S 0 . \quad$ Introduction and Basic Notations}

\section{$\S 0.1$. Introduction}

The deep and interesting feature in the theory of modular varieties beyond the general theory of varieties, is that various important geometric objects are represented in terms of automorphic forms. We already have a literature on such objects. The purpose of this paper is to add yet another new object to them, i.e., automorphic Green functions, which has logarithmic singularity along modular divisors in a modular variety.

The basic data necessary to our construction in this paper is an arithmetic quotient $\Gamma \backslash \mathfrak{D}$ of a bounded symmetric domain $\mathfrak{D}=G / K$ and a divisor on $\Gamma \backslash \mathfrak{D}$ obtained by a modular inbedding $\Gamma_{o} \backslash \mathfrak{D}_{o} \hookrightarrow \Gamma \backslash \mathfrak{D}$, i.e., $\mathfrak{D}_{o}=H / H \cap K$ is a subdomain of complex codimension 1, which is also symmetric and $H$ is a reductive subgroup of the semisimple Lie group $G$ such that the intersection $H \cap K$ of $H$ and a maximal compact subgroup $K$ of $G$ is also maximally compact in $H$. For technical reason, we assume that the pair $(G, H)$ is an affine symmetric pair of rank 1 . Then we have either $(S U(n, 1), S(U(n-1,1) \times U(1)))$ or $(\operatorname{Spin}(2, q), \operatorname{Spin}(2, q-1))$.

To explain the moral of our investigation, we consider the typical case of $G=S U(n, 1)$ and $H=S(U(n-1,1) \times U(1))$. The associated symmetric

Communicated by K. Saito. Received February 6, 2002.

2000 Mathematics Subject Classification(s): 11F55, 14G40.

*Graduate School of Mathematical Sciences, The University of Tokyo, 3-8-1 Komaba Meguro-ku, Tokyo 153-8914, Japan.

e-mail: takayuki@ms.u-tokyo.ac.jp

** Department of Mathematics, Sophia University, Kioi-cho 7-1 Chiyoda-ku, Tokyo 1028554, Japan.

e-mail: tsuzuki@mm.sophia.ac.jp 
domain $\mathfrak{D}=G / K$ is a complex hyperball of dimension $n$, and the orbit $D_{o}$ of the point $o=e K$ of $\mathfrak{D}$ under $H$ is also a complex hyperball. Let $\triangle$ be the $G$-invariant Laplacian on $\mathfrak{D}$. Then there is a unique $H$-invariant solution for the eigenvalue problem

$$
\triangle \phi=\left(n^{2}-s^{2}\right) \phi, \quad s \in \mathbf{C},
$$

which is an $H \times K$-invariant spherical function on $G$. If we admit the singularity along the orbit $D_{o}$, then the same equation has another solution which has the logarithmic singularity along $D_{o}$. Among such solutions, we consider the unique one which has the fastest decay at infinity. We call this the secondary spherical function $\phi_{s}^{(2)}$.

Let $E$ be an imaginary quadratic field and $\Phi$ a non-degenerate Hermitian form of Witt index 1 on an $E$-vector space with signature $(n+, 1-)$. Then $\Phi$ defines a commensurable class of arithmetic lattices in $G$. For such a lattice $\Gamma$, starting with the function $\phi_{s}^{(2)}$, we can construct Poincaré series

$$
G_{s}(z)=\sum_{\gamma \in \Gamma \cap H \backslash \Gamma} \phi_{s}^{(2)}(\gamma z), \quad z \in \Gamma \backslash \mathfrak{D} .
$$

Our main purpose here is to establish some fundamental properties of $G_{s}(z)$. To be more precise, we prove the following:

(i) $G_{s}$ belongs to $L^{p}(\Gamma \backslash \mathfrak{D})$ for any $p$ such that $1 \leqslant p<n$; especially, we have $G_{s} \in L^{2}(\Gamma \backslash \mathfrak{D})$ if $n \geqslant 3$ (Theorem 5.1.1).

(ii) Let $\delta_{D_{o}}$ be the distribution on $\Gamma \backslash \mathfrak{D}$ defined by the integration on $D_{o}$. If $\operatorname{Re}(s)>n$, then we have

$$
\left(\triangle+s^{2}-n^{2}\right) G_{s}(z)=-\delta_{D_{o}}(z)
$$

in the sense of distributions (Corollary 3.2.1).

(iii) Let $n \geqslant 3$. For any compactly supported $C^{\infty}$-function $\varphi(z)$ on $\Gamma \backslash \mathfrak{D}$, the function $s \mapsto\left\langle G_{s}(z), \varphi(z)\right\rangle$ is meromorphically continued to all of $\mathbf{C}$ satisfying a functional equation. Its poles for $\operatorname{Re}(s) \geqslant 0, s \neq 0$ are all simple and are contained in the set of those $s$ such that $n^{2}-s^{2}$ is an eigenvalue of $\triangle$. We have that $\operatorname{Res}_{s=n} G_{s}(z)$ is a constant function (Theorem 6.3.1).

(iv) Let $\tilde{\delta}_{D_{0}}$ be the current of $(1,1)$-type associated with the divisor $D_{o}$. Then the current $\sqrt{-1} \partial \bar{\partial} G_{s}(z)-\pi \tilde{\delta}_{D_{o}}$ is represented concretely by a certain $(1,1)$-form (Theorem 7.8.1). 
For the other case where $G_{\mathbf{R}}=\operatorname{Spin}(2, q), H_{\mathbf{R}}=\operatorname{Spin}(2, q-1)$, we can also show the corresponding statements to (i), (ii) and (iv).

Now we give a brief summary of each section. In the first section, we establish basic settings recalling materials from the reduction theory for a connected semisimple algebraic group over $\mathbf{Q}$, say $G$. We collect several results of Wang [33], which are important in the subsequent discussions. In Section 2, we construct the secondary spherical function $\phi_{s}^{(2)}$ for a class of rank-one affine symmetric space $H_{\mathbf{R}} \backslash G_{\mathbf{R}}$. An automorphic function $G_{s}$ is introduced in Section 3 as a Poincaré series for an arithmetic lattice $\Gamma$ of $G_{\mathbf{R}}$. We establish several basic properties of that Poincaré series in this section. We first show that if $\operatorname{Re}(s)>\rho_{0}$, then the Poincaré series converges absolutely almost everywhere to give an $L^{1}$-function on $\Gamma \backslash G_{\mathbf{R}}$ (Proposition 3.1.1). The first property which we prove for $G_{s}$ is that, as a distribution on $\Gamma \backslash G_{\mathbf{R}}$, it is a fundamental solution of a Poisson equation (Theorem 3.2.1, Corollary 3.2.1). In Section 4, we first introduce a positive-valued $\left(H_{\mathbf{R}}, K\right)$-invariant function $\varphi$ associated to a finite dimensional $H_{\mathbf{R}}$-spherical representation of $G_{\mathbf{R}}$, which we call a gauge function. The main purpose of this section is to have an estimate of the number of cosets $\dot{\gamma} \in \Gamma \cap H_{\mathbf{R}} \backslash \Gamma$ with $\varphi(\gamma x) \leqslant r$ when $x$ varies on a Siegel domain for $\Gamma \backslash G_{\mathbf{R}}$ and $r>0$ (Proposition 4.3.2, Theorem 4.3.1). This estimate is crucial in the proof of meromorphic continuation of $G_{s}$ with respect to $s$ and is actually the most delicate part in $L^{p}$-estimate. The arguments go in a similar line as that of Wang [33]. In Section 5, using the estimate of the counting function obtained in the previous section, we establish an $L^{p}$-estimate of $G_{s}$ for $s$ in a half plane and for $p$ of the form $p=2+\epsilon$ with small $\epsilon>0$ (Theorem 5.1.1). Section 6 is devoted to giving the proof of meromorphicity of $G_{s}$ and to establishing the functional equation for $G_{s}$ in the case when $G_{\mathbf{R}}=S U(n, 1)$. The result is Theorem 6.3.1, which we prove by the method of Miatello-Wallach [20] modifying their argument. But we have to overcome a new difficulty which did not occur in the case of resolvent kernel considerd in [20], that is caused by the fact that the manifold $\Gamma \cap H_{\mathbf{R}} \backslash H_{\mathbf{R}}$ is in general non compact. In the final section, we show that our function $G_{s}$, when considered to be a function on $\Gamma \backslash G_{\mathbf{R}} / K$, enjoys an expected property of the Green current associated with the divisor $\Gamma \cap H_{\mathbf{R}} \backslash H_{\mathbf{R}} / H_{\mathbf{R}} \cap K$ in the sense of Gillet-Soule (Theorem 7.6.1). We note that the proof of Theorem 7.6.1 is essentially a local argument so that no global information on $G_{s}$, such as an $L^{p}$-estimate in the previous sections, is necessary.

Our result is a natural generalization of the classical result for $S U(1,1) \cong$ $S L_{2}(\mathbf{R})$ (cf., [13, Chapter 6, Section 6], [28]). We remark that for elliptic modular case, such Green functions play a key role in the work of Gross-Zagier 
on Heegner points, [10]. Also, we are inspired by the work of Miatello-Wallach who study the Poincaré series obtained from 'bad' Whittaker functions, and also Poincaré series obtained from the reproducing kernel, [19], [20]. The latter object, which is the Green function with point singularity, is in some sense, higher dimensional generalization of the classical Green functions, but to the 'opposite' direction to our generalization. We remark that our construction gives the Green currents of logarithmic type in the sense of Gillet-Soulé $[8,1.3]$ associated with modular divisors modulo still yet unknown behaviour along the boundaries of $\Gamma \backslash G_{\mathbf{R}} / K$. Related to the arithmetic intersection theory of modular varieties, Kudla [22] has some interesting project.

From a different view point, related to the problem of infinite product formulae for automorphic forms, Borcherds [3] constructed certain automorphic forms with logarithmic singularities on the type IV symmetric domains. Generalizing this further, Bruinier constructed some kind of theta lifting $\Psi_{m}(\beta, s)$ of certain Poincaré-Eisenstein series on $S L_{2}(\mathbf{R})$ and elaborated their Fourier expansion explicitly ([5], [4]). Here we note that our Green function $G_{s}$ is closely related to the function $\Psi_{m}(\beta, s)$ investigated by Bruinier. The latter function is related to the older results of Kudla ([21]), Oda ([25]), Rallis-Schiffmann ([27]) and Vigneras ([32]) for $O(n, 2)$.

This paper is just a first step toward the understanding of Green currents associated to modular subvarieties. There are many problems to be exploited around this topic. For example the relation between our result and that of Tong and Wang ([31], [33]), who used some results of Oshima on harmonic analysis of affine symmetric pair, is more substantial than simply technical. We believe that the result of Kobayashi ([17]) strongly suggests that we can push forward our construction for higher codimensional modular cycles. Meanwhile, we note that some fundamental results on the special functions appearing in the various Fourier expansion of non-holomorphic automorphic forms are obtained recently by Ishikawa ([16]) for $S U(2,1)$, Gon ([9]) for $S U(2,2)$ and Ishii ([15]) for $S_{0}(2, q)$. Not only these functions are important as themselves, but also they have the "secondary" ones in their own right. One might investigate the Poincaré series associated with them.

A note written in Japanese based on the talk given by the first-named author at Okayama in 1996 proposes some of the theorems of this paper as conjectures. We thank Dr. Tetsumi Yoshinaga who made the note.

We thank Professor J. Bruinier to point out an error of the constant in the formula (2.5.3) of $\phi_{s}^{(2)}$. We also thank the referee for constructive criticism for this introduction. 


\section{§0.2. Notation}

Let $X$ be a set and $f, g$ two positive real-valued functions defined on $X$. We write

$$
f(x) \prec g(x), \quad x \in X
$$

if there exists a positive constant $c$ such that $f(x) \leqslant c g(x)$ for all $x \in X$.

For any algebraic group $L$ defined over $\mathbf{Q}$ and any $\mathbf{Q}$-algebra $R$, the group of $R$-valued points of $L$ is denoted by $L_{R}$. In particular $L_{\mathbf{R}}$ is the real Lie group consisting of real points of $L$, and $L_{\mathbf{R}}^{\circ}$ is its identity component. Let $X_{\mathbf{Q}}(L)$ denote the group of all rational characters of $L$ defined over $\mathbf{Q}$. For any $\chi \in X_{\mathbf{Q}}(L)$, let $|\chi|: L_{\mathbf{R}} \rightarrow \mathbf{R}^{\times}$be the homomorphism defined by $l \mapsto|\chi(l)|$. We put

$$
{ }^{\circ} L_{\mathbf{R}}=\bigcap_{\chi \in X_{\mathbf{Q}}(L)} \operatorname{ker}|\chi| .
$$

The Lie algebra of real Lie group $L$ is denoted by $\operatorname{Lie}(L)$, and the corresponding German letter $\mathfrak{l}$.

\section{$\S 1$. Preliminaries}

In this section, we recall basic facts on affine symmetric spaces ([12, part II]) and Siegel sets.

\section{§1.1. Algebraic groups}

Let $G$ be a connected semisimple algebraic group defined over $\mathbf{Q}$. Let $\sigma: G \rightarrow G$ be an involution of algebraic groups defined over $\mathbf{Q}$ and $H$ the identity component of the $\sigma$-fixed point subgroup $G^{\sigma}$ of $G$. Hereafter we assume that $H$ has the $\mathbf{Q}$-anisotropic center, or equivalently that $X_{\mathbf{Q}}(H)=\{0\}$.

\section{$\S 1.2$. Real Lie groups}

Let $G$ and $H$ be as above. In what follows, we always assume that $G_{\mathbf{R}}$ is connected. Then the pair $\left(G_{\mathbf{R}}, H_{\mathbf{R}}\right)$ is a semisimple symmetric pair in the usual sense. Let $\theta: G_{\mathbf{R}} \rightarrow G_{\mathbf{R}}$ be a Cartan involution of $G_{\mathbf{R}}$ that commutes with $\sigma$ and $K$ the fixed point subgroup of $\theta$. Then $K$ is a maximal compact subgroup of $G_{\mathbf{R}}$ such that $K_{H}=K \cap H_{\mathbf{R}}$ is maximally compact in $H_{\mathbf{R}}$. 


\section{$\S 1.3$. Root space decomposition, parabolic subgroup and invariant measures}

Let $\mathfrak{g}=\operatorname{Lie}\left(G_{\mathbf{R}}\right)$. Given an involution $\tau$ of $\mathfrak{g}$ and a $\tau$-invariant subspace $V$ of $\mathfrak{g}$, set $V^{\epsilon \tau}=\{X \in V \mid \tau(X)=\epsilon X\}$ for $\epsilon \in\{+,-\}$. Given two involutions $\tau$ and $\sigma$ of $\mathfrak{g}$ mutually commutative, then for $\epsilon, \epsilon^{\prime} \in\{+,-\}$, set $V^{\epsilon \sigma, \epsilon^{\prime} \theta}=$ $V^{\epsilon \sigma} \cap V^{\epsilon^{\prime} \theta}$. We put

$$
\mathfrak{k}=\mathfrak{g}^{\theta}, \quad \mathfrak{p}=\mathfrak{g}^{-\theta}, \quad \mathfrak{h}=\mathfrak{g}^{\sigma}, \quad \mathfrak{q}=\mathfrak{g}^{-\sigma} .
$$

Then $\mathfrak{k}=\operatorname{Lie}(K), \mathfrak{h}=\operatorname{Lie}\left(H_{\mathbf{R}}\right)$ and

$$
\mathfrak{g}=(\mathfrak{k} \cap \mathfrak{h})+(\mathfrak{k} \cap \mathfrak{q})+(\mathfrak{p} \cap \mathfrak{h})+(\mathfrak{p} \cap \mathfrak{q})
$$

is a direct sum decomposition.

Fix a maximal abelian subspace $\mathfrak{a}_{\mathfrak{p}, \mathfrak{q}}$ in $\mathfrak{p} \cap \mathfrak{q}$. In what follows we assume that the split rank of the symmetric pair $\left(G_{\mathbf{R}}, H_{\mathbf{R}}\right)$ is 1 , or equivalently that $\operatorname{dim}_{\mathbf{R}}\left(\mathfrak{a}_{\mathfrak{p}, \mathfrak{q}}\right)=1$.

Let $\Psi=\Psi\left(\mathfrak{a}_{\mathfrak{p}, \mathfrak{q}}, \mathfrak{g}\right)$ be the root system for $\left(\mathfrak{a}_{\mathfrak{p}, \mathfrak{q}}, \mathfrak{g}\right)$. Since we assume that $\operatorname{dim}_{\mathbf{R}}\left(\mathfrak{a}_{\mathfrak{p}, \mathfrak{q}}\right)=1$, this root system is $A_{1}$-type or $B C_{1}$-type. In either case, there exist exactly two short roots in $\Psi$. We take one of them, say $\lambda$, and fix it for once and for all. Let $Y_{0}$ be the vector in $\mathfrak{a}_{\mathfrak{p}, \mathfrak{q}}$ such that $\lambda\left(Y_{0}\right)=1$ and put $\Psi^{+}=\left\{\alpha \in \Psi \mid \alpha\left(Y_{0}\right)>0\right\}$. For any linear form $\alpha$ on $\mathfrak{a}_{\mathfrak{p}, \mathfrak{q}}$, put

$$
\mathfrak{g}_{\alpha}=\left\{X \in \mathfrak{g} \mid\left[Y_{0}, X\right]=\alpha\left(Y_{0}\right) X\right\} .
$$

Then we have the root space decomposition

$$
\mathfrak{g}=\mathfrak{g}_{0}+\sum_{\alpha \in \Psi^{+}}\left(\mathfrak{g}_{\alpha}+\mathfrak{g}_{-\alpha}\right) .
$$

The composite involution $\sigma \theta$ of $\sigma$ and $\theta$ keeps the subspace $\mathfrak{g}_{\alpha}$ invariant to give the direct sum decomposition $\mathfrak{g}_{\alpha}=\mathfrak{g}_{\alpha}^{\sigma \theta}+\mathfrak{g}_{\alpha}^{-\sigma \theta}$. For $i=1,2$ and $\epsilon \in\{+,-\}$, put

$$
m_{i \lambda}^{\epsilon}=\operatorname{dim}_{\mathbf{R}}\left(\mathfrak{g}_{i \lambda}^{\epsilon \sigma \theta}\right)
$$

By definition

$$
\operatorname{dim}_{\mathbf{R}} \mathfrak{g}_{\lambda}=m_{\lambda}^{+}+m_{\lambda}^{-}, \quad \operatorname{dim}_{\mathbf{R}} \mathfrak{g}_{2 \lambda}=m_{2 \lambda}^{+}+m_{2 \lambda}^{-} .
$$

\section{Lemma 1.3.1.}

(1) The space $\mathfrak{g}_{0}=\operatorname{Lie}\left(Z_{G_{\mathbf{R}}}\left(\mathfrak{a}_{\mathfrak{p}, \mathfrak{q}}\right)\right)$ is $\sigma$-stable and $\theta$-stable. We have $\mathfrak{g}_{0}^{-\sigma,-\theta}=$ $\mathfrak{a}_{\mathfrak{p}, \mathfrak{q}} \cdot$ 
(2) Let $\alpha \in \Psi^{+}$. The subspace $\mathfrak{g}_{\alpha}+\mathfrak{g}_{-\alpha}$ is $\sigma$-stable and $\theta$-stable. For any $\epsilon, \epsilon^{\prime} \in\{+,-\}$, the following maps are $\mathbf{R}$-linear isomorphisms:

$$
\begin{aligned}
& \mathfrak{g}_{\alpha} \cong\left(\mathfrak{g}_{\alpha}+\mathfrak{g}_{-\alpha}\right)^{\epsilon \sigma}, \quad X \mapsto X+\epsilon \sigma X, \\
& \mathfrak{g}_{\alpha} \cong\left(\mathfrak{g}_{\alpha}+\mathfrak{g}_{-\alpha}\right)^{\epsilon^{\prime} \theta}, \quad X \mapsto X+\epsilon^{\prime} \theta X, \\
& \mathfrak{g}_{\alpha}^{\epsilon \epsilon^{\prime} \sigma \theta} \stackrel{\cong}{\longrightarrow}\left(\mathfrak{g}_{\alpha}+\mathfrak{g}_{-\alpha}\right)^{\epsilon \sigma, \epsilon^{\prime} \theta}, \quad X \mapsto X+\epsilon \sigma X=X+\epsilon^{\prime} \theta X .
\end{aligned}
$$

Proof. The second part of (1) follows from the maximality of $\mathfrak{a}_{\mathfrak{p}, \mathfrak{q}}$ in $\mathfrak{p} \cap \mathfrak{q}$. The statements in (2) are proved in [29, Lemma 7].

Now let $\mathfrak{a}_{\mathfrak{p}, \mathfrak{h}}$ be a maximal abelian subspace of $\mathfrak{g}_{0}^{\sigma,-\theta}$ and put $\mathfrak{a}_{\mathfrak{p}}=\mathfrak{a}_{\mathfrak{p}, \mathfrak{q}}+$ $\mathfrak{a}_{\mathfrak{p}, \mathfrak{h}}$. Then $\mathfrak{a}_{\mathfrak{p}}$ is a maximal abelian subspace of $\mathfrak{p}$ containing $\mathfrak{a}_{\mathfrak{p}, \mathfrak{q}}$. Let $\Phi_{\mathbf{R}}$ be the restricted root system for $\left(\mathfrak{a}_{\mathfrak{p}}, \mathfrak{g}\right)$ and ${ }^{0} \Phi_{\mathbf{R}}$ that for $\left(\mathfrak{a}_{\mathfrak{p}}, \mathfrak{g}_{0}\right)$. For a positive system ${ }^{0} \Phi_{\mathbf{R}}^{+}$of ${ }^{0} \Phi_{\mathbf{R}}$, the union

$$
\Phi_{\mathbf{R}}^{+}=\left\{\beta \in \Phi_{\mathbf{R}} \mid \beta\left(Y_{0}\right)>0\right\} \cup{ }^{0} \Phi_{\mathbf{R}}^{+}
$$

is a positive system of $\Phi_{\mathbf{R}}$, compatible with $\Psi^{+}$. Let $P_{\mathfrak{p}}$ be the minimal parabolic subgroup of $G_{\mathbf{R}}$ with a Levi subgroup $Z_{G_{\mathbf{R}}}\left(\mathfrak{a}_{\mathfrak{p}}\right)$ corresponding to the positive system $\Phi_{\mathbf{R}}^{+}$. Now we introduce the $\sigma$-minimal parabolic subgroup $Q_{\mathfrak{p}, \mathfrak{q}}$ containing $P_{\mathfrak{p}}$, which is important in the representation theory of the symmetric space $G_{\mathbf{R}} / H_{\mathbf{R}}$. It is defined by

$$
Q_{\mathfrak{p}, \mathfrak{q}}=Z_{G_{\mathbf{R}}}\left(\mathfrak{a}_{\mathfrak{p}, \mathfrak{q}}\right) U, \quad U=\exp \left(\mathfrak{g}_{\lambda}+\mathfrak{g}_{2 \lambda}\right)
$$

with the unipotent radical $U$.

Let $\rho_{0}$ be the real number defined by

$$
2 \rho_{0}=\operatorname{tr}\left(\operatorname{ad}\left(Y_{0}\right) \mid \operatorname{Lie}(U)\right),
$$

or explicitly $2 \rho_{0}=m_{\lambda}^{+}+m_{\lambda}^{-}+2 m_{2 \lambda}^{+}+2 m_{2 \lambda}^{-}$.

We normarize various invariant measures as follows: Firstly we fix a Haar meaure $d h$ of $H_{\mathbf{R}}$. Then we can normalize the Haar measure of $G_{\mathbf{R}}$ so that the integration formula

$$
\int_{G_{\mathbf{R}}} \varphi(g) d g=\int_{H_{\mathbf{R}}} d h \int_{K} d k \int_{0}^{\infty} \varphi\left(h \exp \left(t Y_{0}\right) k\right) \gamma_{\mathfrak{h} \backslash \mathfrak{g}}(t) d t, \quad \varphi \in C_{\mathrm{c}}\left(G_{\mathbf{R}}\right)
$$

is valid, [12, p. 110, Theorem 2.5]. Here $d k$ is the Haar measure of $K$ with total mass one, $d t$ is the usual Lebesgue measure of $\mathbf{R}$ and $\gamma_{\mathfrak{h} \backslash \mathfrak{g}}(t)$ is given by

$$
\gamma_{\mathfrak{h} \backslash \mathfrak{g}}(t)=(\sinh (t))^{m_{\lambda}^{+}}(\cosh (t))^{m_{\lambda}^{-}}\left(2^{-1} \sinh (2 t)\right)^{m_{2 \lambda}^{+}}(\cosh (2 t))^{m_{2 \lambda}^{-}} .
$$


Let $\Gamma$ be an arithmetic subgroup of $G_{\mathbf{Q}}$ and $\Gamma_{H}=\Gamma \cap H_{\mathbf{R}}$. Let $d \dot{h}$ be the quotient measure of $d h$ by the counting measure of $\Gamma_{H}$. As the quotient measure of $d g$ by the counting measure on the discrete group $\Gamma$, we get a right $G_{\mathbf{R}^{-}}$ invariant measure $d \dot{g}$ on $\Gamma \backslash G_{\mathbf{R}}$.

\section{§1.4. Siegel sets}

Let $P_{G}$ be a minimal parabolic $\mathbf{Q}$-subgroup of $G$ with the unipotent radical $N_{G}$; let $A_{G}$ be a maximal $\mathbf{Q}$-split torus in the radical of $P_{G}$. For a positive real number $t>0$, put

$$
A_{G, \mathbf{R}}^{+}(t)=\left\{a \in A_{G, \mathbf{R}}^{\circ} \mid a^{\alpha} \geqslant t, \forall \alpha \in \Delta_{G}\right\},
$$

where $\Delta_{G}$ denotes the set of simple roots in $\Phi\left(A_{G}, G\right)$ with respect to the positive system determined by $P_{G}$. For a relatively compact subset $\omega$ of ${ }^{\circ} P_{G, \mathbf{R}}$ and a positive real number $t$, put

$$
\mathcal{S}_{\omega, t}=\omega A_{G, \mathbf{R}}^{+}(t) K .
$$

Any subset of the form $\mathcal{S}_{\omega, t}$ is called a Siegel set of $G_{\mathbf{R}}$.

Since $G_{\mathbf{R}}={ }^{\circ} P_{G, \mathbf{R}} A_{G, \mathbf{R}}^{\circ} K$, any element $g \in G_{\mathbf{R}}$ can be written as $g=p a k$ with $p \in{ }^{\circ} P_{G, \mathbf{R}}, a \in A_{G, \mathbf{R}}^{\circ}$ and $k \in K$. Although this decomposition of $g$ is not

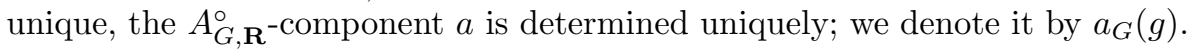
Let $d s, d a$ and $d k$ be Haar measures of ${ }^{\circ} P_{G, \mathbf{R}}, A_{G, \mathbf{R}}^{\circ}$ and $K$ respectively; if we choose $d s$ and $d a$ suitably then the following integration formula is valid:

$$
\int_{G_{\mathbf{R}}} f(g) d g=\int_{{ }^{\circ} P_{G, \mathbf{R}}} \int_{A_{G, \mathbf{R}}^{\circ}} \int_{K} f(s a k) a^{-2 \rho_{G}} d k d a d s, \quad f \in C_{\mathbf{c}}\left(G_{\mathbf{R}}\right),
$$

where $2 \rho_{G}$ is the rational character of $A_{G}$ defined by

$$
a^{2 \rho_{G}}=\operatorname{det}\left(\operatorname{Ad}(a) \mid \operatorname{Lie}\left(N_{G}\right)\right), \quad a \in A_{G} .
$$

Lemma 1.4.1. For any $\epsilon>0, t>0, l \in \mathbf{N}$ and $u \in \mathbf{N}$, we have

$$
\int_{A_{G, \mathbf{R}}^{+}(t)} a^{-\epsilon \rho_{G}}\left(1+\left|\log a^{\rho_{G}}\right|^{l}\right)^{u} d a<+\infty .
$$

Proof. Let $\left\{Y_{\alpha} \mid \alpha \in \Delta_{G}\right\}$ be the basis of $\operatorname{Lie}\left(A_{G, \mathbf{R}}\right)$ that is dual to $\Delta_{G}$ with respect to the natural pairing $\operatorname{Lie}\left(A_{G, \mathbf{R}}\right) \times \mathfrak{a}_{G}^{*} \rightarrow \mathbf{R}$. Here $\mathfrak{a}_{G}^{*}=$ 
$X_{\mathbf{Q}}\left(A_{G}\right) \otimes_{\mathbf{z}} \mathbf{R}$. Then $c_{\alpha}=\left\langle Y_{\alpha}, 2 \rho_{G}\right\rangle$ is positive for any $\alpha \in \Delta_{G}$. The integral in question is dominated by a finite sum of integrals of the form

$$
\prod_{\alpha \in \Delta_{G}} \int_{\log t}^{\infty} e^{-\epsilon c_{\alpha} t_{\alpha}} t_{\alpha}^{m_{\alpha}} d t_{\alpha}, \quad m_{\alpha} \in \mathbf{N}
$$

each of which is finite because $\epsilon>0$ and $c_{\alpha}>0$.

Let $\Gamma$ be an arithmetic subgroup of $G_{\mathbf{Q}}$. By the reduction theory, there exists a finite subset $C$ of $G_{\mathbf{Q}}$ and a Siegel set $\mathcal{S}=\mathcal{S}_{\omega, t}$ such that

(a) $G_{\mathbf{R}}=\Gamma C \mathcal{S}$

(b) the set $\{\gamma \in \Gamma \mid \mathcal{S} \cap \gamma \mathcal{S} \neq \emptyset\}$ is finite.

See Borel $[1,13.1$. Théorème, 15.4. Théorème] for example. A Siegel set having this property is call a Siegel domain for $\Gamma \backslash G_{\mathbf{R}}$.

Lemma 1.4.2. Let $f: G_{\mathbf{R}} \rightarrow \mathbf{R}_{+}$be a left $\Gamma$-invariant positive-valued measurable function. Let $\mathcal{S}$ be a Siegel domain for $\Gamma \backslash G_{\mathbf{R}}$. Then the integral $\int_{\mathcal{S}} f(x) d x$ is finite if and only if the integral $\int_{\Gamma \backslash G_{\mathbf{R}}} f(x) d \dot{x}$ is finite.

Proof. This is a consequence of the fact that the natural projection $\mathcal{S} \rightarrow$ $\Gamma \backslash G_{\mathbf{R}}$ is a finite covering map.

Let $P_{H}, N_{H}, A_{H}$ and $2 \rho_{H}$ be the counterparts for $H$ of $P_{G}, N_{G}, A_{G}$ and $2 \rho_{G}$ for $G$. Since $\Gamma_{H}=\Gamma \cap H_{\mathbf{R}}$ is an arithmetic subgroup of $H_{\mathbf{Q}}$, we can take a Siegel domain $\mathcal{S}_{H}=\mathcal{S}_{\omega_{H}, t_{H}}$ for $\Gamma_{H} \backslash H_{\mathbf{R}}$ with respect to the minimal parabolic $P_{H}$ and its split component $A_{H}$. Here $\omega_{H}$ is a compact subset of ${ }^{\circ} P_{H, \mathbf{R}}$ and $t_{H}$ is a positive number. Let $C_{H}$ be a finite subset of $H_{\mathbf{Q}}$ such that $H_{\mathbf{R}}=\Gamma_{H} C_{H} \mathcal{S}_{H}$.

Since we assume that $G$ is semisimple the volume of the quotient space $\Gamma \backslash G_{\mathbf{R}}$ is finite. As for $H$, since $X_{\mathbf{Q}}(H)=\{0\}$ as we assumed in 1.1, the volume of $\Gamma_{H} \backslash H_{\mathbf{R}}$ is finite also. See [1, 13.2. Corollaire].

\section{$\S 1.5$. Fundamental set for $\Gamma \cap H_{\mathbf{R}} \backslash \boldsymbol{G}_{\mathbf{R}}$}

We extract some basic facts from Wang [33]. Let $P_{H}$ and $A_{H}$ be as in 1.4. It is known that $\Phi_{G}=\Phi\left(A_{H}, G\right)$, the set of eigen-characters of $A_{H}$ in $\operatorname{Lie}(G)$, is a root system that containes $\Phi_{H}=\Phi\left(A_{H}, H\right)$ as a subroot system. Let $\Phi^{(j)}, 1 \leqslant j \leqslant m$ be the totality of positive systems of $\Phi\left(A_{H}, G\right)$ containing $\Phi_{H}^{+}=\Phi\left(A_{H}, P_{H}\right)$. For each $j$, let $2 \rho_{H}^{(j)} \in X_{\mathbf{Q}}\left(A_{H}\right)$ be the sum of roots in 
$\Phi^{(j)}$ with multiplicities and $\Delta^{(j)}$ the set of simple roots for $\Phi^{(j)}$. Let $\mathfrak{a}_{H}^{*}=$ $X_{\mathbf{Q}}\left(A_{H}\right) \otimes_{\mathbf{Z}} \mathbf{R}$ and ${ }^{+} \mathfrak{a}_{H}^{*}$ the closed cone in $\mathfrak{a}_{H}^{*}$ generated by the roots in $\Phi_{H}^{+}$.

The proofs in [33] suggest that it is convenient to introduce a number $\tau_{\mathbf{Q}}(G, \sigma)$, that plays an important role in what follows.

Definition 1.5.1. We define a real number $\tau=\tau_{\mathbf{Q}}(G, \sigma)$ by

$$
\tau=\sup \left\{\eta \in[0,2] \mid 2 \rho_{H}-\eta \rho_{H}^{(j)} \in{ }^{+} \mathfrak{a}_{H}^{*},(1 \leqslant j \leqslant m)\right\} .
$$

For given positive numbers $t>0$ and $t_{0}>0$, set

$$
A_{H, \mathbf{R}}^{(j)}\left(t ; t_{0}\right)=\left\{a_{H} \in A_{H, \mathbf{R}}^{+}(t) \mid a_{H}^{\alpha}>t_{0}, \forall \alpha \in \Delta^{(j)}\right\} .
$$

Let $\Gamma$ be an arithmetic subgroup of $G_{\mathbf{Q}}$ and put $\Gamma_{H}=\Gamma \cap H_{\mathbf{R}}$.

Proposition 1.5.1. Let $\mathcal{S}_{H}=\mathcal{S}_{\omega_{H}, t_{H}}$ be a Siegel domain for $\Gamma_{H} \backslash H_{\mathbf{R}}$ such that $H_{\mathbf{R}}=\Gamma_{H} C_{H} \mathcal{S}_{H}$ with $C_{H}$ a finite subset of $H_{\mathbf{Q}}$. Then there exists a positive number $t_{0}>t_{H}$ such that $\mathcal{S}_{H}$ is covered by $m$ open subsets

$$
\mathcal{S}_{H}^{(j)}=\omega_{H} A_{H, \mathbf{R}}^{(j)}\left(t_{H} ; t_{0}\right) K_{H}
$$

with $1 \leqslant j \leqslant m$. Put $A_{\mathfrak{p}, \mathfrak{q}}^{+}=\left\{\exp \left(t Y_{0}\right) \mid t \geqslant 0\right\}$. Then we have the following decomposition of $G_{\mathbf{R}}$ :

$$
G_{\mathbf{R}}=\bigcup_{j=1}^{m} \Gamma_{H} C_{H} \mathcal{S}_{H}^{(j)} A_{\mathfrak{p}, \mathfrak{q}}^{+} K .
$$

Proof. See [33, 5.9, p. 344].

Proposition 1.5.2. $\quad$ Retain the notations in Proposition 1.5.1. Let $\mathcal{S}=$ $\mathcal{S}_{\omega, t}$ be a Siegel domain for $\Gamma \backslash G_{\mathbf{R}}$ such that $G_{\mathbf{R}}=\Gamma C \mathcal{S}$ with $C$ a finite subset of $G_{\mathbf{Q}}$. Let $1 \leqslant j \leqslant m$ and $\mathcal{N}$ a compact subset of $G_{\mathbf{R}}$.

Then we have

$$
e^{-t \rho_{0}} a_{H}(h)^{-\rho_{H}^{(j)}} \prec a_{G}(g)^{-\rho_{G}}
$$

as long as

$$
\begin{aligned}
& \gamma \kappa g u=\delta \kappa_{H} h \exp \left(t Y_{0}\right) k, \\
& \quad \gamma \in \Gamma, \kappa \in C, g \in \mathcal{S}, u \in \mathcal{N}, \delta \in \Gamma_{H}, \kappa_{H} \in C_{H}, h \in \mathcal{S}_{H}^{(j)}, t \geqslant 0, k \in K .
\end{aligned}
$$

Proof. See [33, Proposition 5.9]. 


\section{$\S 2 . \quad$ Spherical Functions}

In this section, firstly we recall the 'ordinary' spherical functions on $H_{\mathbf{R}} \backslash G_{\mathbf{R}} / K$. Afterwards we consider other functions defined on $H_{\mathbf{R}} \backslash\left(G_{\mathbf{R}}-\right.$ $\left.H_{\mathbf{R}} K\right) / K$, which are eigenfunctions with respect to the Casimir operator with singularity along $H_{\mathbf{R}} K$.

\section{§2.1. Radial part of the Casimir operator}

Let $B: \mathfrak{g} \times \mathfrak{g} \rightarrow \mathbf{R}$ be the Killing form of $\mathfrak{g}$. Using the decomposition (1.3.1), we have $B\left(Y_{0}, Y_{0}\right)=2 \operatorname{dim}_{\mathbf{R}} \mathfrak{g}_{\lambda}+8 \operatorname{dim}_{\mathbf{R}} \mathfrak{g}_{2 \lambda}$. Let $\Omega$ be the normalized Casimir element of $\mathfrak{g}$ corresponding to the bilinear form

$$
\langle X, Y\rangle=\frac{1}{B\left(Y_{0}, Y_{0}\right)} B(X, Y), \quad X, Y \in \mathfrak{g} .
$$

For any $C^{\infty}$-function $f$ on $G_{\mathbf{R}}$ (or on an open subset of $G_{\mathbf{R}}$ ), an element $X \in \mathfrak{g}$ acts by

$$
(f * X)(g)=\left.\frac{d}{d t}\right|_{t=0} f(g \exp (t X)), \quad g \in G_{\mathbf{R}} .
$$

This action is extendable to the universal enveloping algebra of $\mathfrak{g}$. We have the radial part of $\Omega$ :

Proposition 2.1.1. Let $S=H_{\mathbf{R}} K$. For any $\varphi \in C^{\infty}\left(H_{\mathbf{R}} \backslash\left(G_{\mathbf{R}}-\right.\right.$ $S) / K)$ put $\phi(t)=\varphi\left(\exp \left(t Y_{0}\right)\right), t \in \mathbf{R}$. Then we have

$$
\begin{aligned}
(\varphi * \Omega)\left(\exp \left(t Y_{0}\right)\right) & =\gamma_{\mathfrak{h} \backslash \mathfrak{g}}(t)^{-1} \frac{d}{d t}\left(\gamma_{\mathfrak{h} \backslash \mathfrak{g}}(t) \frac{d}{d t} \phi(t)\right) \\
& =\left(\frac{d^{2}}{d t^{2}}+R_{\mathfrak{h} \backslash \mathfrak{g}}(t) \frac{d}{d t}\right) \phi(t), \quad t>0,
\end{aligned}
$$

where

$$
\begin{aligned}
R_{\mathfrak{h} \backslash \mathfrak{g}}(t) & =\gamma_{\mathfrak{h} \backslash \mathfrak{g}}(t)^{-1} \gamma_{\mathfrak{h} \backslash \mathfrak{g}}^{\prime}(t) \\
& =m_{\lambda}^{+} \operatorname{coth}(t)+m_{\lambda}^{-} \tanh (t)+2 m_{2 \lambda}^{+} \operatorname{coth}(2 t)+2 m_{2 \lambda}^{-} \tanh (2 t) .
\end{aligned}
$$

Proof. The first equality [7, Eq. (4.12)] immediately gives the second one. 


\section{$\S 2.2$. Principal series for $G_{\mathrm{R}} / H_{\mathrm{R}}$ and spherical functions}

Recall the $H_{\mathbf{R}}$-spherical principal series of $G_{\mathbf{R}}$. For the parabolic subgroup $Q_{\mathfrak{p}, \mathfrak{q}}$ defined in Subsection 1.3 and a complex number $s$, let us consider a quasicharacter $\chi_{s}: Q_{\mathfrak{p}, \mathfrak{q}} \rightarrow \mathbf{C}^{\times}$given by

$$
\chi_{s}(q)=|\operatorname{det}(\operatorname{Ad}(q) \mid \operatorname{Lie}(U))|^{s /\left(2 \rho_{0}\right)}, \quad q \in Q_{\mathfrak{p}, \mathfrak{q}} .
$$

Then $\chi_{s}\left(\exp \left(t Y_{0}\right)\right)=e^{t s}$. Let $\mathcal{H}_{s}^{\infty}$ be the space of all $C^{\infty}$-functions $\varphi: G_{\mathbf{R}} \rightarrow \mathbf{C}$ such that

$$
\varphi(q g)=\chi_{s+\rho_{0}}(q) \varphi(g), \quad q \in Q_{\mathfrak{p}, \mathfrak{q}}, g \in G_{\mathbf{R}} .
$$

The action of $G_{\mathbf{R}}$ through the right translation on $\mathcal{H}_{s}^{\infty}$ is a smoothly induced representation $\operatorname{Ind}_{Q_{\mathfrak{p}, \mathfrak{q}}}^{G_{\mathbf{R}}}\left(\chi_{s}\right)$ of $G_{\mathbf{R}}$ which we denote by $\pi_{s}^{\infty}$ simply.

Lemma 2.2.1. For any $\varphi \in \mathcal{H}_{s}^{\infty}$, we have

$$
\pi_{s}^{\infty}(\Omega) \varphi=\left(s^{2}-\rho_{0}^{2}\right) \varphi
$$

Proof. See [18, Proposition 8.22].

Now we recall briefly how to construct a $G_{\mathbf{R}}$-intertwining operator from $\pi_{s}^{\infty}$ to $C^{\infty}\left(H_{\mathbf{R}} \backslash G_{\mathbf{R}}\right)$ using the open double coset $H_{\mathbf{R}} Q_{\mathfrak{p}, \mathfrak{q}}$. For a given $s \in \mathbf{C}$, we define a function $\xi_{s}: G_{\mathbf{R}} \rightarrow \mathbf{C}$ by putting

$$
\begin{aligned}
\xi_{s}(h q) & =\chi_{s-\rho_{0}}(q), \quad h \in H_{\mathbf{R}}, q \in Q_{\mathfrak{p}, \mathfrak{q}}, \\
\xi_{s}(g) & =0, \quad g \in G_{\mathbf{R}}-H_{\mathbf{R}} Q_{\mathfrak{p}, \mathfrak{q}} .
\end{aligned}
$$

It is known that if $\operatorname{Re}(s)>\rho_{0}$, then the function $\xi_{s}$ is continuous on $G_{\mathbf{R}},[12$, p. 145, Proposition 6.1]; hence the integral

$$
\mathcal{P}_{s}(\varphi: g)=\int_{K} \xi_{s}\left(k^{-1}\right) \varphi(k g) d k, \quad g \in G_{\mathbf{R}}
$$

converges absolutely for any $\varphi \in \mathcal{H}_{s}^{\infty}$. Here $d k$ is the Haar measure of $K$ with the total mass 1 . It can be proved that the function $g \mapsto \mathcal{P}_{s}(\varphi: g)$ belongs to the space $C^{\infty}\left(H_{\mathbf{R}} \backslash G_{\mathbf{R}}\right)$ and $\varphi \mapsto \mathcal{P}_{s}(\varphi)$ gives a $G_{\mathbf{R}}$-intertwining map from $\pi_{s}^{\infty}$ to $C^{\infty}\left(H_{\mathbf{R}} \backslash G_{\mathbf{R}}\right)$.

Let $\varphi_{s}^{0}$ be the $K$-spherical vector in $\mathcal{H}_{s}^{\infty}$ such that $\varphi_{s}^{0}(e)=1$. The integral

$$
c(s)=\int_{V} \varphi_{s}^{0}(v) d v, \quad \operatorname{Re}(s)>\rho_{0}
$$


gives the $c$-function for the principal series $\pi_{s}^{\infty}$, where $V=\theta U$ and $d v$ is a suitably normalized Haar measure of that group. Let us introduce the $\left(H_{\mathbf{R}}, K\right)$ spherical function $\phi_{s}^{(1)}$ for $\pi_{s}^{\infty}$ by

$$
\phi_{s}^{(1)}(g)=\frac{1}{c(s)} \mathcal{P}_{s}\left(\varphi_{s}^{0}: g\right), \quad g \in G_{\mathbf{R}} .
$$

What we need is the following property of $\phi_{s}^{(1)}$.

Proposition 2.2.1. Let $s \in \mathbf{C}$ with $\operatorname{Re}(s)>\rho_{0}$. Then

(1) $\phi_{s}^{(1)} \in C^{\infty}\left(H_{\mathbf{R}} \backslash G_{\mathbf{R}} / K\right)$ and

$$
\phi_{s}^{(1)} * \Omega=\left(s^{2}-\rho_{0}^{2}\right) \phi_{s}^{(1)} .
$$

(2) We have

$$
\lim _{t \rightarrow+\infty} e^{t\left(\rho_{0}-s\right)} \phi_{s}^{(1)}\left(\exp \left(t Y_{0}\right)\right)=1 .
$$

Proof. (1) is a consequence of the intertwining property of $\mathcal{P}_{s}$ and Lemma 2.2.1. As for (2), see [12, p. 167, Proposition 7.7].

\section{§2.3. Differential equation of spherical function}

For a given complex number $s$, we consider an ordinary differential equation of second order:

$$
\text { （ ) } \quad \frac{d^{2} u}{d t^{2}}+R_{\mathfrak{h} \backslash \mathfrak{g}}(t) \frac{d u}{d t}+\left(\rho_{0}^{2}-s^{2}\right) u=0 .
$$

Here

$$
\begin{aligned}
R_{\mathfrak{h} \backslash \mathfrak{g}}(t) & =m_{\lambda}^{+} \operatorname{coth}(t)+m_{\lambda}^{-} \tanh (t)+2 m_{2 \lambda}^{+} \operatorname{coth}(2 t)+2 m_{2 \lambda}^{-} \tanh (2 t), \\
2 \rho_{0} & =m_{\lambda}^{+}+m_{\lambda}^{-}+2 m_{2 \lambda}^{+}+2 m_{2 \lambda}^{-} .
\end{aligned}
$$

In view of Propositions 2.1.1 and 2.2.1 (1), the spherical function $\phi_{s}^{(1)}$ constructed in the previous subsection provides us with a solution $u^{(1)}(t)=\phi_{s}^{(1)}$ $\left(\exp \left(t Y_{0}\right)\right)$ of $(\star)$ that is defined on $\mathbf{R}$ and is smooth there.

\section{§2.4. Secondary spherical function}

\section{Proposition 2.4.1.}

(1) For any $s \in \mathbf{C}, t=0$ is a regular singular point of the differential equation $(\star)$. The characteristic exponents of $(\star)$ at $t=0$ are $\left\{0,1-m_{\lambda}^{+}-m_{2 \lambda}^{+}\right\}$. 
(2) The following three statements are equivalent.

(a) The differential equation ( $\star$ ) admits a local solution around $t=0$ of the form $u(t)=\log (t)\left(1+\sum_{n=1}^{\infty} a_{n} t^{n}\right)+\sum_{n=0}^{\infty} b_{n} t^{n}$ with $\sum a_{n} t^{n}$ and $\sum b_{n} t^{n}$ power series convergent in a neighborhood of $t=0$.

(b) $\operatorname{dim}\left(G_{\mathbf{R}} / K\right)=\operatorname{dim}\left(H_{\mathbf{R}} / K_{H}\right)+2$.

(c) $m_{\lambda}^{+}+m_{2 \lambda}^{+}=1$.

Proof. (1) Since the function $R_{\mathfrak{h} \backslash \mathfrak{g}}(t)$ has a simple pole at $t=0$ and since $s^{2}-\rho_{0}^{2}$ is a constant, the criterion of regular singularity implies that $t=0$ is a regular singular point of $(\star)$. The residue of $R_{\mathfrak{h} \backslash \mathfrak{g}}(t)$ at $t=0$ is

$$
\begin{aligned}
\operatorname{Res}_{t=0} R_{\mathfrak{h} \backslash \mathfrak{g}}(t) & =m_{\lambda}^{+} \lim _{t \rightarrow 0} t \operatorname{coth}(t)+2 m_{2 \lambda}^{+} \lim _{t \rightarrow 0} t \operatorname{coth}(2 t) \\
& =m_{\lambda}^{+}+m_{2 \lambda}^{+} .
\end{aligned}
$$

Thus the indicial equation of $(\star)$ at $t=0$ becomes

$$
\xi^{2}+\left(m_{\lambda}^{+}+m_{2 \lambda}^{+}-1\right) \xi=0 .
$$

This completes the proof of (1). Next we prove (2). The equivalence of (a) and (c) follows from the classical theory on fundamental solutions of second order differential equations of Fuchsian type. See [34, Chapter V, pp. 194-201] for example. The equivalence of (b) and (c) is a consequnce of the root space decomposition (1.3.1). Indeed we have

$$
\operatorname{dim}\left(G_{\mathbf{R}} / K\right)-\operatorname{dim}\left(H_{\mathbf{R}} / K_{H}\right)=\operatorname{dim}_{\mathbf{R}}(\mathfrak{p})-\operatorname{dim}_{\mathbf{R}}(\mathfrak{p} \cap \mathfrak{h})=\operatorname{dim}_{\mathbf{R}}(\mathfrak{p} \cap \mathfrak{q}) .
$$

By taking the $-\sigma$ - and $-\theta$-invariant part of the decomposition (1.3.1) and noting that $\mathfrak{g}_{0}^{-\sigma,-\theta}=\mathfrak{a}_{\mathfrak{p}, \mathfrak{q}}$, we have

$$
\mathfrak{p} \cap \mathfrak{q}=\mathfrak{a}_{\mathfrak{p}, \mathfrak{q}}+\left(\mathfrak{g}_{\lambda}+\mathfrak{g}_{-\lambda}\right)^{-\sigma,-\theta}+\left(\mathfrak{g}_{2 \lambda}+\mathfrak{g}_{-2 \lambda}\right)^{-\sigma,-\theta}
$$

on one hand. On the other hand Lemma 1.3.1 (2) gives

$$
\operatorname{dim}_{\mathbf{R}}\left(\mathfrak{g}_{\alpha}+\mathfrak{g}_{-\alpha}\right)^{-\sigma,-\theta}=\operatorname{dim}_{\mathbf{R}} \mathfrak{g}_{\alpha}^{\sigma \theta}=m_{\alpha}^{+}
$$

for $\alpha \in \Psi^{+}$. Hence we have $\operatorname{dim}_{\mathbf{R}}(\mathfrak{p} \cap \mathfrak{q})=m_{\lambda}^{+}+m_{2 \lambda}^{+}+1$. Consequently we have the equality

$$
\operatorname{dim}\left(G_{\mathbf{R}} / K\right)-\operatorname{dim}\left(H_{\mathbf{R}} / K_{H}\right)=m_{\lambda}^{+}+m_{2 \lambda}^{+}+1 .
$$

By this formula, the equivalence of (b) and (c) is now clear.

In the rest of this paper we assume the conditions (a), (b) and (c) in Proposition 2.4.1 are satisfied. 
Proposition 2.4.2. Assume the conditions in Proposition 2.4.1 (2). Put $S=H_{\mathbf{R}} K$. Let $s$ be a complex number with $\operatorname{Re}(s)>\rho_{0}$. Then there exists a unique function $\phi_{s}^{(2)}: G_{\mathbf{R}}-S \rightarrow \mathbf{C}$ that satisfies the following conditions:

(a) The function $\phi_{s}^{(2)}$ is $C^{\infty}$ away from $S$ and

$$
\phi_{s}^{(2)}(h g k)=\phi_{s}^{(2)}(g), \quad h \in H_{\mathbf{R}}, g \in G_{\mathbf{R}}-S, k \in K .
$$

(b) $\phi_{s}^{(2)} * \Omega(g)=\left(s^{2}-\rho_{0}^{2}\right) \phi_{s}^{(2)}(g), \quad g \in G_{\mathbf{R}}-S$.

(c) There exists a positive $\delta$ such that $\phi_{s}^{(2)}\left(\exp \left(t Y_{0}\right)\right)-\log (t)$ is bounded on $0<t<\delta$. In particular, the $\left(H_{\mathbf{R}}, K\right)$-invariant function $\phi_{s}^{(2)}$ is locally integrable on $G_{\mathbf{R}}$.

(d) We have

$$
\phi_{s}^{(2)}\left(\exp \left(t Y_{0}\right)\right)=O\left(e^{-\left(\operatorname{Re}(s)+\rho_{0}\right) t}\right), \quad(t \rightarrow+\infty) .
$$

Proof. We extend the argument of [6, 4.1. Satz.] slightly. For $r>0$ and $c \in \mathbf{C}$, put

$$
\Delta_{r}^{*}(c)=\{z \in \mathbf{C}|0<| z-c \mid<r\} .
$$

The condition (a) means that it is sufficient to define the $A_{\mathfrak{p}, \mathfrak{q}}$-radial part $\phi_{s}^{(2)} \mid A_{\mathfrak{p}, \mathfrak{q}}$ of $\phi_{s}^{(2)}$. Put $r=e^{t}$. Then the condition (b) is equivalent to the equation

$$
\frac{d^{2} w}{d r^{2}}+p(r) \frac{d w}{d r}-\frac{s^{2}-\rho_{0}^{2}}{r^{2}} w=0
$$

with

$$
p(r)=\frac{1}{r}\left(1+m_{\lambda}^{+} \frac{r^{2}+1}{r^{2}-1}+m_{\lambda}^{-} \frac{r^{2}-1}{r^{2}+1}+2 m_{2 \lambda}^{+} \frac{r^{4}+1}{r^{4}-1}+2 m_{2 \lambda}^{-} \frac{r^{4}-1}{r^{4}+1}\right) .
$$

The condition (c) for $w(r)=\phi_{s}^{(2)}\left(\exp \left(t Y_{0}\right)\right)$ means that $w(r)$ has logarithmic singularity $\log (r-1)$ at $r=1$. Our first task is to show that there exists a non-zero solution of (2.4.1) with this singularity. We divide the proof into several steps.

$<$ Local solution at $r=\infty>$

By a computation, we have that the characteristic exponents of (2.4.1) at $r=\infty$ are $\rho_{0}-s$ and $\rho_{0}+s$. By the theory on fundamental solutions, we 
can take two linearly independent local solutions of (2.4.1) around $r=\infty$, say $w_{\infty}^{(1)}(r)$ and $w_{\infty}^{(2)}(r)$, of the form

$$
\begin{aligned}
& w_{\infty}^{(2)}(r)=r^{-\rho_{0}-s} \sum_{n=0}^{\infty} a_{n} r^{-n}, \\
& w_{\infty}^{(1)}(r)=r^{-\rho_{0}+s} \sum_{n=1}^{\infty} b_{n} r^{-n}+c w_{\infty}^{(2)}(r) \log \left(\frac{1}{r}\right),
\end{aligned}
$$

where $a_{n}, b_{n}, c \in \mathbf{C}$ and $\sum a_{n} z^{n}, \sum b_{n} z^{n}$ converges in a neighbourhood of $z=0$.

Claim 2.4.1. The local solutions $w_{\infty}^{(i)}(r), i=1,2$ are analytically continued to solutions on $\Delta_{1}^{*}(\infty)=\{r \in \mathbf{C}|| r \mid>1\}$.

Proof. In the $z$-coordinate, the equation (2.4.1) becomes

$$
\frac{d^{2} w}{d z^{2}}+\left(\frac{2}{z}-\frac{p\left(z^{-1}\right)}{z^{2}}\right) \frac{d w}{d z}-\frac{s^{2}-\rho_{0}^{2}}{z^{2}} w=0
$$

The possible singularities of $2 z^{-1}-z^{-2} p\left(z^{-1}\right)$ except $z=0$ are at eighth roots of unity lying on the unit circle $|z|=1$. Hence the local solutions $w_{\infty}^{(1)}\left(z^{-1}\right)$ and $w_{\infty}^{(2)}\left(z^{-1}\right)$ of (2.4.2) around $z=0$ are continued to solutions on the punctured unit disc $\Delta_{1}^{*}(0)$.

$<$ Local solutions at $r=1>$

Next we consider the situation around $r=1$. From the assumption the indicial equation of (2.4.1) at $r=1$ has double zero 0 . Hence we can take a fundamental system of local solutions of (2.4.1) around $r=1$ of the form

$$
\begin{aligned}
& w_{1}^{(1)}(r)=1+\sum_{n=1}^{\infty} c_{n}(r-1)^{n}, \\
& w_{1}^{(2)}(r)=\sum_{n=0}^{\infty} d_{n}(r-1)^{n}+w_{1}^{(1)}(r) \log (r-1),
\end{aligned}
$$

where $\sum d_{n}(r-1)^{n}$ and $\sum c_{n}(r-1)^{n}$ are convergent on the domain $\Delta_{\delta}^{*}(1)$ with small $\delta>0$.

Now we consider the coefficients of connection between two systems $\left\{w_{1}^{(i)} \mid i=1,2\right\}$ and $\left\{w_{\infty}^{(i)} \mid i=1,2\right\}$ on the domain $\Delta_{1}^{*}(\infty) \cap \Delta_{\delta}^{*}(1)$ with small $\delta>0$. 


\section{Claim 2.4.2.}

(i) There exists a non zero constant $c_{1}$ such that

$$
w_{1}^{(1)}\left(e^{t}\right)=c_{1} \phi_{s}^{(1)}\left(\exp \left(t Y_{0}\right)\right), \quad t>0
$$

where $\phi_{s}^{(1)}$ is the spherical function introduced in 2.2 .

(ii) There exist constants $p$ and $q$ with $q \neq 0$ such that

$$
w_{\infty}^{(2)}(r)=p w_{1}^{(1)}(r)+q w_{1}^{(2)}(r), \quad 1<r<\delta .
$$

In particular $w_{\infty}^{(2)}(r)$ has logarithmic singularity at $r=1$.

Proof. (i) Since $u^{(1)}(r)=\phi_{s}^{(1)}\left(\exp \left((\log r) Y_{0}\right)\right)$ is a $C^{\infty}$-solution on $r>0$ of (2.4.1) (see 2.3), there exist complex numbers $c_{1}$ and $c_{2}$ such that

$$
u^{(1)}(r)=c_{1} w_{1}^{(1)}(r)+c_{2} w_{1}^{(2)}(r), \quad 1<r<\delta .
$$

Since $u^{(1)}$ is smooth at $r=1$ and since $w_{1}^{(2)}(r)=\log (r-1)+O(1)$ around $r=1$, we have $c_{2}=0$ and $c_{1} \neq 0$. This completes the proof of (i). (ii) Since $w_{1}^{(i)}, i=1,2$ forms a system of fundamental solutions on $\Delta_{\delta}^{*}(1)$, we can write $w_{\infty}^{(2)}(r)$ as a linear combination

$$
w_{\infty}^{(2)}(r)=p w_{1}^{(1)}(r)+q w_{1}^{(2)}(r)
$$

on $\mathbf{R} \cap \Delta_{1}^{*}(\infty) \cap \Delta_{\delta}^{*}(1)$. Assume $q=0$. Then we have

$$
w_{\infty}^{(2)}(r)=p w_{1}^{(1)}(r)
$$

for $1<r<\delta$. Since $w_{\infty}^{(2)}(r)$ and $w_{1}^{(1)}(r)$ are both analytic on $r>1$, the identity (2.4.3) is valid on $r>1$. Now consider the limit

$$
\lim _{r \rightarrow+\infty} r^{\rho_{0}-s} w_{\infty}^{(2)}(r)=p \lim _{r \rightarrow+\infty} r^{\rho_{0}-s} w_{1}^{(1)}(r) .
$$

Since $r^{\rho_{0}-s} w_{\infty}^{(2)}(r)=O\left(r^{-2 \operatorname{Re}(s)}\right)$ as $r \rightarrow+\infty$, we have that the left hand side of (2.4.4) is zero. As for the right hand side, it is $p c_{1}$ by (i) and the property of $\phi_{s}^{(1)}$ presented in Proposition 2.2.1 (2). Consequently we have $0=p c_{1}$, or $p=0$. Since $q$ is assumed to be zero, we have $w_{\infty}^{(2)}(r)=0$ identically. This is absurd.

Since $q \neq 0$, we can take a constant $c_{3} \in \mathbf{C}$ so that

$$
c_{3}\left(p w_{1}^{(1)}(r)+q w_{1}^{(2)}(r)\right)=\log (r-1)+O(1), \quad r \rightarrow 1+0 .
$$


Now set $u^{(2)}(r)=c_{3} w_{\infty}^{(2)}(r), r>1$. Since the map $(h, r, k) \mapsto h \exp \left((\log r) Y_{0}\right) k$ is a submersive surjection from $H_{\mathbf{R}} \times\{r \mid r>1\} \times K$ onto the open set $G_{\mathbf{R}}-S$, there exists a unique $C^{\infty}$-function $\phi_{s}^{(2)}: G_{\mathbf{R}}-S \rightarrow \mathbf{C}$ such that

$$
\phi_{s}^{(2)}\left(h \exp \left(t Y_{0}\right) k\right)=u^{(2)}\left(e^{t}\right), \quad h \in H_{\mathbf{R}}, t>0, k \in K .
$$

This completes the construction of $\phi_{s}^{(2)}$. The uniqueness of the function $\phi_{s}^{(2)}$ having the properties (a) to (d) is confirmed as follows: Assume we are given a function $\phi$ with the same property as that of $\phi_{s}^{(2)}$. Firstly, the conditions (a) and (b) imply that $u(r)=\phi\left((\log r) Y_{0}\right)$ is a $C^{\infty}$-solution of the equation (2.4.1). The condition (d) means that if we write $u(r)$ as a linear combination of $w_{\infty}^{(1)}$ and $w_{\infty}^{(2)}$ on $r>1$, then the coefficient of $w_{\infty}^{(1)}$ has to be zero. Hence $u(r)=c_{4} w_{\infty}^{(2)}(r)$ with a constant $c_{4}$. Taking the condition (c) into consideration, we have $c_{3}=c_{4}$, or equivalently $u(r)=u^{(2)}(r)$.

Corollary 2.4.1. Let $s \in \mathbf{C}$ with $\operatorname{Re}(s)>\rho_{0}$. Then there exist positive numbers $R_{1}$ and $R_{2}$, power series with positive radius of convergences $\sum_{n=0}^{\infty} a_{n} z^{n}, \sum_{n=1}^{\infty} e_{n}^{\prime} z^{n}$ and $\sum_{n=0}^{\infty} e_{n}^{\prime \prime} z^{n}$ such that

$$
\begin{aligned}
& \phi_{s}^{(2)}\left(\exp \left(t Y_{0}\right)\right)=e^{-\left(s+\rho_{0}\right) t} \sum_{n=0}^{\infty} a_{n} e^{-t n}, \quad\left(t>R_{1}\right), \\
& \phi_{s}^{(2)}\left(\exp \left(t Y_{0}\right)\right)=\log (t)\left(1+\sum_{n=1}^{\infty} e_{n}^{\prime} t^{n}\right)+\sum_{n=0}^{\infty} e_{n}^{\prime \prime} t^{n}, \quad\left(0<t<R_{2}\right) .
\end{aligned}
$$

Proof. Indeed, as we have seen in the proof of Proposition 2.4.2, $\phi_{s}^{(2)}\left(\exp \left(t Y_{0}\right)\right)$ is given by $c_{3} w_{\infty}^{(2)}\left(e^{t}\right)$ in a neighborhood of $t=\infty$ and by $p w_{1}^{(1)}\left(e^{t}\right)+q w_{1}^{(2)}\left(e^{t}\right)$ with $q \neq 0$ in a neighborhood of $t=0$.

Remark 2.4.1. The coefficients $a_{n}$ of $w_{\infty}^{(2)}$ in the proof of Proposition 2.4 .2 are rational functions of $s$ that are holomorphic on $\operatorname{Re}(s)>\rho_{0}$; from this it is proved that $\phi_{s}^{(2)}$ depends on $s$ for $\operatorname{Re}(s)>\rho_{0}$ holomorphically.

\section{$\S 2.5$. Secondary spherical functions as hypergeometric series}

In view of the list of affine symmetric spaces of split rank one [12, p. 185], we can confirm that a semisimple symmetric space of rank one $G_{\mathbf{R}} / H_{\mathbf{R}}$ satisfying the condition in Proposition 2.4.1 (2) is isomorphic to one of the following. 
(i) $G_{\mathbf{R}} / H_{\mathbf{R}} \cong S U(n, 1) / S(U(n-1,1) \times U(1))$ with $n \geqslant 1 ; m_{2 \lambda}^{+}=1, m_{\lambda}^{+}=$ $m_{2 \lambda}^{-}=0, m_{\lambda}^{-}=2 n-2$ and $\rho_{0}=n$.

(ii) $G_{\mathbf{R}} / H_{\mathbf{R}} \cong \operatorname{Spin}(2, q) / \operatorname{Spin}(2, q-1)$ with $q \geqslant 2 ; m_{\lambda}^{+}=1, m_{2 \lambda}^{+}=m_{2 \lambda}^{-}=0$, $m_{\lambda}^{-}=q-1$ and $\rho_{0}=q / 2$.

We have an explicit formula of spherical functions in terms of Gaussian hypergeometric series. By change of variables $z=-\sinh ^{2}(t)$, we can transform the differential equation $(\star)$ to the Gaussian hypergeometric differential equation:

$$
z(1-z) \frac{d^{2} u}{d z^{2}}+\left(1-\left(\rho_{0}+1\right) z\right) \frac{d u}{d z}-\frac{\rho_{0}^{2}-s^{2}}{4} u=0
$$

We have

$$
\begin{aligned}
\phi_{s}^{(1)}\left(\exp \left(t Y_{0}\right)\right)= & \frac{\Gamma\left(\frac{s+\rho_{0}}{2}\right) \Gamma\left(\frac{s-\rho_{0}}{2}+1\right)}{\Gamma(s+1)} \\
& \times{ }_{2} F_{1}\left(\frac{\rho_{0}+s}{2}, \frac{\rho_{0}-s}{2} ; 1 ;-\sinh ^{2}(t)\right) \\
\phi_{s}^{(2)}\left(\exp \left(t Y_{0}\right)\right)= & -\frac{1}{2} \frac{\Gamma\left(\frac{s+\rho_{0}}{2}\right) \Gamma\left(\frac{s-\rho_{0}}{2}+1\right)}{\Gamma(s+1)} \\
& \times(\cosh (t))^{-\left(s+\rho_{0}\right)} 2 \\
& \times F_{1}\left(\frac{s+\rho_{0}}{2}, \frac{s-\rho_{0}}{2}+1 ; s+1 ; \frac{1}{\cosh ^{2}(t)}\right)
\end{aligned}
$$

We briefly explain that $\phi_{s}^{(2)}\left(\exp \left(t Y_{0}\right)\right)$ is indeed given by the formula above: Verify first that the right hand side of $(2.5 .3)$, say $\phi(t)$, satisfies the differential equation (2.5.1). Since ${ }_{2} F_{1}(a, b ; c ; z)=O(1)$ near $z=0$, the behavior of $\phi(t)$ around $t=\infty$ is controled by that of $(2 \cosh (t))^{-\left(s+\rho_{0}\right)}$, that is $O\left(e^{-\left(s+\rho_{0}\right) t}\right)$ for $t$ large enough. Using [24, formula (9.7.5), p. 257], we have $\phi(t)=\log (t)+O(1)$ near $t=0$. By Proposition 2.4.2, we have $\phi(t)=\phi_{s}^{(2)}\left(\exp \left(t Y_{0}\right)\right)$ as desired.

\section{§3. Poincaré Series Constructed from Secondary Spherical Functions}

Recall that the involution $\sigma: G \rightarrow G$ is defined over $\mathbf{Q}$; hence the algebraic group $H$, the connected component of $G^{\sigma}$, is also defined over $\mathbf{Q}$. We assume that $X_{\mathbf{Q}}(H)=\{0\}$. We choose a Cartan involution $\theta: G_{\mathbf{R}} \rightarrow G_{\mathbf{R}}$ commutative 
with $\sigma$ and put $K=G_{\mathbf{R}}^{\theta}$; we say such a $K$ is admissible with respect to $H$. Then $K_{H}=H_{\mathbf{R}} \cap K$ is a maximal compact subgroup of $H_{\mathbf{R}}$. Our assumptions on $G_{\mathbf{R}}, H_{\mathbf{R}}$ and $K$ are as below.

(i) $G_{\mathbf{R}}$ is connected.

(ii) $\left(G_{\mathbf{R}}, H_{\mathbf{R}}\right)$ is of rank one, or $\operatorname{dim}_{\mathbf{R}} \mathfrak{a}_{\mathfrak{p}, \mathfrak{q}}=1$.

(iii) $\operatorname{dim}\left(G_{\mathbf{R}} / K\right)=\operatorname{dim}\left(H_{\mathbf{R}} / K_{H}\right)+2$, or $m_{\lambda}^{+}+m_{2 \lambda}^{+}=1$.

Throughout this section, $\Gamma$ denotes an arithmetic subgroup of $G_{\mathbf{Q}}$ and $\Gamma_{H}=\Gamma \cap H_{\mathbf{R}}$.

\section{§3.1. Poincaré series}

Let $s$ be a complex number with $\operatorname{Re}(s)>\rho_{0}$ and $\phi_{s}^{(2)}$ the function in Proposition 2.4.2. We introduce a Poincaré series obtained from the secondary spherical function $\phi_{s}^{(2)}$ :

$(\bullet)$

$$
{ }^{\Gamma} G_{s}(x)=\sum_{\gamma \in \Gamma_{H} \backslash \Gamma} \phi_{s}^{(2)}(\gamma x), \quad x \in G_{\mathbf{R}} .
$$

From now on, we write $G_{s}(x)$ in place of ${ }^{\Gamma} G_{s}(x)$ for simplicity.

Proposition 3.1.1. $\quad$ There exists a left $\Gamma$-invariant zero set $\tilde{S}$ of $G_{\mathbf{R}}$ containing $S=H_{\mathbf{R}} K$ such that the series $(\downarrow)$ converges absolutely for $x \in$ $G_{\mathbf{R}}-\tilde{S}$. The function $x \mapsto G_{s}(x)$, that is defined on $G_{\mathbf{R}}-\tilde{S}$, gives a measurable function on $\Gamma \backslash\left(G_{\mathbf{R}}-\tilde{S}\right)$ with finite $L^{1}$-norm.

Proof. We first note that the set $\Gamma S$ contains all subsets of the form $\gamma^{-1} H_{\mathbf{R}} K$ with $\gamma \in \Gamma$ along which the function $\phi_{s}^{(2)}(\gamma x)$ has singularities and that $\Gamma S$ is measure zero since $S$ is a zero set and $\Gamma$ is a countable set. Hence if $x \in G_{\mathbf{R}}-\Gamma S$ we can discuss the convergence of the series $(\checkmark)$ at $x$, since all terms of the series are well defined. Using the integration formula (1.3.2), we have

$$
\begin{gathered}
\int_{\Gamma \backslash G_{\mathbf{R}}}\left(\sum_{\gamma \in \Gamma_{H} \backslash \Gamma}\left|\phi_{s}^{(2)}(\gamma x)\right|\right) d \dot{x} \\
=\int_{\Gamma_{H} \backslash G_{\mathbf{R}}}\left|\phi_{s}^{(2)}(x)\right| d \dot{x}
\end{gathered}
$$




$$
\begin{aligned}
& =\int_{H_{\mathbf{R}} \backslash G_{\mathbf{R}}} d \dot{x} \int_{\Gamma_{H} \backslash H_{\mathbf{R}}}\left|\phi_{s}^{(2)}(h x)\right| d \dot{h} \\
& =\int_{H_{\mathbf{R}} \backslash G_{\mathbf{R}}} d \dot{x} \int_{\Gamma_{H} \backslash H_{\mathbf{R}}}\left|\phi_{s}^{(2)}(x)\right| d \dot{h} \\
& =\operatorname{vol}\left(\Gamma_{H} \backslash H_{\mathbf{R}}\right) \int_{H_{\mathbf{R}} \backslash G_{\mathbf{R}}}\left|\phi_{s}^{(2)}(x)\right| d \dot{x} \\
& =\operatorname{vol}\left(\Gamma_{H} \backslash H_{\mathbf{R}}\right) \int_{0}^{+\infty}\left|\phi_{s}^{(2)}\left(\exp \left(t Y_{0}\right)\right)\right| \gamma_{\mathfrak{h} \backslash \mathfrak{g}}(t) d t .
\end{aligned}
$$

As we have remarked in 1.4, $\operatorname{vol}\left(\Gamma_{H} \backslash H_{\mathbf{R}}\right)$ is finite. By Corollary 2.4 .1 and the explicit form of $\gamma_{\mathfrak{h} \backslash \mathfrak{g}}(t)$ given in (1.3.3), we have

$$
\left|\phi_{s}^{(2)}\left(\exp \left(t Y_{0}\right)\right)\right| \prec e^{-\left(\operatorname{Re}(s)+\rho_{0}\right) t}, \quad \gamma_{\mathfrak{h} \backslash \mathfrak{g}}(t) \prec e^{2 \rho_{0} t}
$$

for $t>1$. Hence the integral

$$
\int_{1}^{\infty}\left|\phi_{s}^{(2)}\left(\exp \left(t Y_{0}\right)\right)\right| \gamma_{\mathfrak{h} \backslash \mathfrak{g}}(t) d t
$$

on the interval $[1, \infty)$, which is dominated by a constant times of the integral of $e^{\left(-\operatorname{Re}(s)+\rho_{0}\right) t}$ on the interval $t>1$, is finite for $\operatorname{Re}(s)>\rho_{0}$. In order to bound the integral

$$
\int_{0}^{1}\left|\phi_{s}^{(2)}\left(\exp \left(t Y_{0}\right)\right)\right| \gamma_{\mathfrak{h} \backslash \mathfrak{g}}(t) d t
$$

on the interval $(0,1]$, by Corollary 2.4 .1 and (1.3.3), we can use

$$
\left|\phi_{s}^{(2)}\left(\exp \left(t Y_{0}\right)\right)\right| \prec|\log (t)|, \quad \gamma_{\mathfrak{h} \backslash \mathfrak{g}}(t) \prec t^{m_{\lambda}^{+}+m_{2 \lambda}^{+}}=t
$$

for $0<t<1$. Hence the integral above is dominated by a constant times of the integral of $|\log (t)| t$ on $0<t<1$, that is obviously finite. Summing up the argument so far, we have

$$
\int_{\Gamma \backslash G_{\mathbf{R}}}\left(\sum_{\gamma \in \Gamma_{H} \backslash \Gamma}\left|\phi_{s}^{(2)}(\gamma x)\right|\right) d \dot{x}<+\infty .
$$

Let $\tilde{S}$ be the union of $\Gamma S$ and the set of $x \in G_{\mathbf{R}}-\Gamma S$ such that $\sum_{\gamma \in \Gamma_{H} \backslash \Gamma}\left|\phi_{s}^{(2)}(\gamma x)\right|=+\infty$. Then the set $\tilde{S}$ is a left $\Gamma$-invariant zero set having the property in the proposition.

For an $s$ with $\operatorname{Re}(s)>\rho_{0}$ the function $\dot{x} \mapsto G_{s}(x)$ defined on $\Gamma \backslash\left(G_{\mathbf{R}}-\widetilde{S}\right)$ has finite $L^{1}$-norm; it defines a locally integrable function on $\Gamma \backslash G_{\mathbf{R}}$, i.e., an 
element of $L_{\text {loc }}^{1}\left(\Gamma \backslash G_{\mathbf{R}}\right)$. Hence we can consider $G_{s}(x)$ as a distribution on $\Gamma \backslash G_{\mathbf{R}}$ by the formula:

$$
\left\langle G_{s}, \varphi\right\rangle=\int_{\Gamma \backslash G_{\mathbf{R}}} G_{s}(x) \varphi(x) d \dot{x}, \quad \varphi \in C_{\mathrm{c}}^{\infty}\left(\Gamma \backslash G_{\mathbf{R}}\right) .
$$

Remark 3.1.1. The fact that the distribution $G_{s}$ for $\operatorname{Re}(s)>\rho_{0}$ depends holomorphically on $s$ is proved by a standard argument.

Proposition 3.1.2. $\quad$ Let $s \in \mathbf{C}$ with $\operatorname{Re}(s)>\rho_{0}$. Then we have

$$
\int_{\Gamma \backslash G_{\mathbf{R}}} G_{s}(x) d \dot{x}=\frac{\operatorname{vol}\left(\Gamma_{H} \backslash H_{\mathbf{R}}\right)}{\rho_{0}^{2}-s^{2}} .
$$

Proof. By a similar computation as in the proof of Proposition 3.1.1, we have

$$
\int_{\Gamma \backslash G_{\mathbf{R}}} G_{s}(x) d \dot{x}=\operatorname{vol}\left(\Gamma_{H} \backslash H_{\mathbf{R}}\right) \int_{0}^{\infty} \phi_{s}^{(2)}\left(\exp \left(t Y_{0}\right)\right) \gamma_{\mathfrak{h} \backslash \mathfrak{g}}(t) d t
$$

Using the differential equation $(\star)$,

$$
\begin{aligned}
\int_{0}^{\infty} & \phi_{s}^{(2)}\left(\exp \left(t Y_{0}\right)\right) \gamma_{\mathfrak{h} \backslash \mathfrak{g}}(t) d t \\
= & \frac{1}{s^{2}-\rho_{0}^{2}} \int_{0}^{\infty}\left(\gamma_{\mathfrak{h} \backslash \mathfrak{g}}(t) \frac{d^{2}}{d t^{2}}+\gamma_{\mathfrak{h} \backslash \mathfrak{g}}^{\prime}(t) \frac{d}{d t}\right) \phi_{s}^{(2)}\left(\exp \left(t Y_{0}\right)\right) d t \\
= & \frac{1}{s^{2}-\rho_{0}^{2}} \int_{0}^{\infty} \frac{d}{d t}\left(\gamma_{\mathfrak{h} \backslash \mathfrak{g}}(t) \frac{d}{d t} \phi_{s}^{(2)}\left(\exp \left(t Y_{0}\right)\right)\right) d t \\
= & \frac{1}{s^{2}-\rho_{0}^{2}}\left[\gamma_{\mathfrak{h} \backslash \mathfrak{g}}(t) \frac{d}{d t} \phi_{s}^{(2)}\left(\exp \left(t Y_{0}\right)\right)\right]_{0}^{\infty} \\
= & \frac{-1}{s^{2}-\rho_{0}^{2}} .
\end{aligned}
$$

The last equality is obtained by the estimate (2.4.5) for large $t>0$ and also by the expansion around $t=0$

$$
\gamma_{\mathfrak{h} \backslash \mathfrak{g}}(t) \frac{d}{d t} \phi_{s}^{(2)}\left(\exp \left(t Y_{0}\right)\right)=1+O(t)(1+\log (t)),
$$

that follows from (2.4.6) and (1.3.3). 


\section{§3.2. Differential equation for $\boldsymbol{G}_{s}$}

The subject of this subsection is to verify that the Poincaré series introduced in the previous subsection are fundamental solutions of a Poisson equation. Let $\mathcal{S}$ be a Siegel domain for $\Gamma \backslash G_{\mathbf{R}}$ such that $G_{\mathbf{R}}=\Gamma C \mathcal{S}$ with a finite subset $C$ of $G_{\mathbf{Q}}$. Recall the number $\tau_{\mathbf{Q}}(G, \sigma)$ in Definition 1.5.1.

Theorem 3.2.1. Assume that $\tau=\tau_{\mathbf{Q}}(G, \sigma)>1$. Moreover let $\varphi \in$ $C^{\infty}\left(\Gamma \backslash G_{\mathbf{R}}\right)$ be a function satisfying the condition:

There exists a $\delta$ with $0<\delta<\tau-1$ such that for any $0<\epsilon<1-\delta$ and any $X \in U\left(\mathfrak{g}_{\mathbf{C}}\right)$

$$
|(\varphi * X)(\kappa g)| \prec a_{G}(g)^{(2-\epsilon) \rho_{G}}, \quad \kappa \in C, g \in \mathcal{S} .
$$

Then given a complex number $s$ with $\operatorname{Re}(s)>(\tau+1) \rho_{0}$, we have

$$
\int_{\Gamma_{H} \backslash H_{\mathbf{R}}} \int_{K}|\varphi(h k)| d k d \dot{h}<+\infty
$$

$$
\int_{\Gamma \backslash G_{\mathbf{R}}}\left|G_{s}(x)\left(\varphi *\left(\Omega+\rho_{0}^{2}-s^{2}\right)\right)(x)\right| d \dot{x}<+\infty,
$$

(3) and

$$
\int_{\Gamma \backslash G_{\mathbf{R}}} G_{s}(x)\left(\varphi *\left(\Omega+\rho_{0}^{2}-s^{2}\right)\right)(x) d \dot{x}=\int_{\Gamma_{H} \backslash H_{\mathbf{R}}} \int_{K} \varphi(h k) d k d \dot{h} .
$$

Proof. (1) For any continuous function $f$ on $\Gamma \backslash G_{\mathbf{R}}$, put

$$
J(f: x)=\int_{\Gamma_{H} \backslash H_{\mathbf{R}}} \int_{K} f(h x k) d k d \dot{h}, \quad x \in G_{\mathbf{R}} .
$$

We examine the convergence of the integral $J(|\varphi|: x)$. By the assumption, for any $0<\epsilon<1-\delta$, there exists a positive constant $c_{0}$ such that

$$
|\varphi(\kappa g)| \leqslant c_{0} \cdot a_{G}(g)^{(2-\epsilon) \rho_{G}}, \quad g \in \mathcal{S}, \kappa \in C .
$$

By Proposition 1.5.1, the Siegel domain $\mathcal{S}_{H}=\mathcal{S}_{\omega_{H}, t_{H}}$ for $\Gamma_{H} \backslash H_{\mathbf{R}}$ is a union of $m$ subsets $\mathcal{S}_{H}^{(j)}=\omega_{H} A_{H, \mathbf{R}}^{(j)}\left(t_{H} ; t_{0}\right) K_{H}$ with $j=1, \ldots, m$. By Proposition 1.5.2, there exists a positive constant $c_{1}$ such that if

$$
\gamma \kappa g=h \exp \left(t Y_{0}\right) k
$$


with $\gamma \in \Gamma, \kappa \in C, g \in \mathcal{S}, h \in \mathcal{S}_{H}^{(j)}, t \geqslant 0$ and $k \in K$, then the inequality

$$
a_{G}(g)^{\rho_{G}} \leqslant c_{1} \cdot a_{H}(h)^{\rho_{H}^{(j)}} e^{t \rho_{0}}
$$

holds. Hence using (3.2.1) we have

$$
\left|\varphi\left(h \exp \left(t Y_{0}\right) k\right)\right|=|\varphi(\kappa g)| \leqslant c_{0} \cdot a_{G}(g)^{(2-\epsilon) \rho_{G}} \leqslant c_{1} c_{0}\left(e^{t \rho_{0}} a_{H}(h)^{\rho_{H}^{(j)}}\right)^{2-\epsilon} .
$$

By intergration, we have

$$
\begin{aligned}
& J\left(|\varphi|: \exp \left(t Y_{0}\right)\right) \\
& \quad \leqslant \sum_{j=1}^{m} \int_{\mathcal{S}_{H}^{(j)}} \int_{K}\left|\varphi\left(h \exp \left(t Y_{0}\right) k\right)\right| d k d h \\
& \quad \leqslant c_{0} c_{1} \cdot e^{(2-\epsilon) t \rho_{0}}\left(\int_{\omega_{H}} d s_{1} \int_{K_{H}} d k_{1}\right) \sum_{j=1}^{m} \int_{A_{H, \mathbf{R}}^{(j)}\left(t_{H} ; t_{0}\right)} a_{H}^{(2-\epsilon) \rho_{H}^{(j)}-2 \rho_{H}} d a_{H}
\end{aligned}
$$

with $d s_{1}$ and $d k_{1}$ Haar measures of the groups ${ }^{\circ} P_{H, \mathbf{R}}$ and $K_{H}$ respectively. Now since $2-\tau<1-\delta$, we can choose $\epsilon>0$ so that $2-\tau<\epsilon<1-\delta$; then by definition of the number $\tau$ we can take $\eta$ so that $2 \rho_{H}-\eta \rho_{H}^{(j)} \in{ }^{+} \mathfrak{a}_{H}^{*}$ for $j=1, \ldots, m$ and $2-\epsilon<\eta \leqslant \tau$. We have

$$
\int_{A_{H, \mathbf{R}}^{(j)}\left(t_{H} ; t_{0}\right)} a_{H}^{(2-\epsilon) \rho_{H}^{(j)}-2 \rho_{H}} d a_{H} \leqslant t_{H}^{r} \int_{A_{H, \mathbf{R}}^{+}\left(t_{H}\right)} a_{H}^{\left((2-\epsilon) \eta^{-1}-1\right) 2 \rho_{H}} d a_{H}
$$

with some constant $r \in \mathbf{R}$. Hence thanks to Lemma 1.4.1, we see that the last integral is finite because of our choice of $\epsilon$ and $\eta$. Summing up the argument so far, we have

$$
J\left(|\varphi|: \exp \left(t Y_{0}\right)\right) \prec e^{(2-\epsilon) t \rho_{0}}, \quad t \geqslant 0
$$

for any $\epsilon$ with $2-\tau<\epsilon<1-\delta$. In particular the integral $J(|\varphi|: e)$ is finite.

(2) Put

$$
\psi(g)=\varphi *\left(\Omega+\rho_{0}^{2}-s^{2}\right)(g), \quad g \in G_{\mathbf{R}} .
$$

We have

$$
\begin{aligned}
\int_{\Gamma \backslash G_{\mathbf{R}}}\left|G_{s}(x)\right||\psi(x)| d \dot{x} \\
\quad \leqslant \int_{\Gamma \backslash G_{\mathbf{R}}}\left(\sum_{\gamma \in \Gamma_{H} \backslash \Gamma}\left|\phi_{s}^{(2)}(\gamma x)\right||\psi(\gamma x)|\right) d \dot{x} \\
=\int_{\Gamma_{H} \backslash G_{\mathbf{R}}}\left|\phi_{s}^{(2)}(x)\right||\psi(x)| d \dot{x}
\end{aligned}
$$




$$
\begin{aligned}
& =\int_{H_{\mathbf{R}} \backslash G_{\mathbf{R}}}\left(\int_{\Gamma_{H} \backslash H_{\mathbf{R}}}\left|\phi_{s}^{(2)}(h x)\right||\psi(h x)| d \dot{h}\right) d \dot{x} \\
& =\int_{H_{\mathbf{R}} \backslash G_{\mathbf{R}}}\left|\phi_{s}^{(2)}(x)\right|\left(\int_{\Gamma_{H} \backslash H_{\mathbf{R}}}|\psi(h x)| d \dot{h}\right) d \dot{x} \\
& =\int_{0}^{\infty}\left|\phi_{s}^{(2)}\left(\exp \left(t Y_{0}\right)\right)\right| \gamma_{\mathfrak{h} \backslash \mathfrak{g}}(t) J\left(|\psi|: \exp \left(t Y_{0}\right)\right) d t .
\end{aligned}
$$

Here to show the last equality we use the integration formula (1.3.1). Now from (1.3.3) we have

$$
\gamma_{\mathfrak{h} \backslash \mathfrak{g}}(t) \prec e^{2 t \rho_{0}}, \quad t>0 .
$$

Since $\psi$ has the same property as $\varphi$, from the proof of (1) we have

$$
J\left(|\psi|: \exp \left(t Y_{0}\right)\right) \prec e^{(2-\epsilon) t \rho_{0}}, \quad t>0
$$

for any $\epsilon$ with $2-\tau<\epsilon<1-\delta$. By condition (d) of Proposition 2.4.2, there exists a positive constant $R$ such that

$$
\left|\phi_{s}^{(2)}\left(\exp \left(t Y_{0}\right)\right)\right| \prec e^{-\left(\operatorname{Re}(s)+\rho_{0}\right) t}, \quad t>R .
$$

Hence we have

$$
\begin{aligned}
\int_{R}^{\infty} & \left|\phi_{s}^{(2)}\left(\exp \left(t Y_{0}\right)\right)\right| \gamma_{\mathfrak{h} \backslash \mathfrak{g}}(t) J\left(|\psi|: \exp \left(t Y_{0}\right)\right) d t \\
& \prec \int_{R}^{\infty} e^{(2-\epsilon) t \rho_{0}} e^{2 \rho_{0} t} e^{-\left(\operatorname{Re}(s)+\rho_{0}\right) t} d t \\
& \leqslant \int_{R}^{\infty} e^{\left(-\operatorname{Re}(s)+(\tau+1) \rho_{0}\right) t} d t .
\end{aligned}
$$

If $\operatorname{Re}(s)>(\tau+1) \rho_{0}$, the last integral is finite. Next we consider the integral on the finite interval $0<t \leqslant R$. By the condition (c) of Proposition 2.4.2, we have

$$
\left|\phi_{s}^{(2)}\left(\exp \left(t Y_{0}\right)\right)\right| \prec|\log (t)|, \quad 0<t<R .
$$

From (1.3.3), we have an estimate of the form

$$
\gamma_{\mathfrak{h} \backslash \mathfrak{g}}(t) \prec t^{m_{\lambda}^{+}+m_{2 \lambda}^{+}}, \quad 0<t<R .
$$


Hence, noting that $J\left(|\psi|: \exp \left(t Y_{0}\right)\right)$ is bounded on a finite interval, we have

$$
\begin{aligned}
& \int_{0}^{R}\left|\phi_{s}^{(2)}\left(\exp \left(t Y_{0}\right)\right)\right| \gamma_{\mathfrak{h} \backslash \mathfrak{g}}(t) J\left(|\psi|: \exp \left(t Y_{0}\right)\right) d t \\
& \quad \prec \int_{0}^{R}|\log (t)| t^{m_{\lambda}^{+}+m_{2 \lambda}^{+}} d t .
\end{aligned}
$$

Since $m_{\lambda}^{+}+m_{2 \lambda}^{+}=1$, this integral is finite. This completes the proof of (2).

(3) By a similar computation as (3.2.3) in the proof of (2), we have the identity

$$
\begin{aligned}
& \int_{\Gamma \backslash G_{\mathbf{R}}} G_{s}(x)\left(\varphi *\left(\Omega+\rho_{0}^{2}-s^{2}\right)\right)(x) d \dot{x} \\
& \quad=\int_{0}^{\infty} \phi_{s}^{(2)}\left(\exp \left(t Y_{0}\right)\right) \gamma_{\mathfrak{h} \backslash \mathfrak{g}}(t) J\left(\varphi *\left(\Omega+\rho_{0}^{2}-s^{2}\right): \exp \left(t Y_{0}\right)\right) d t .
\end{aligned}
$$

As we have seen in the proof of (1), the integral $J\left(\varphi: \exp \left(t Y_{0}\right)\right)$ is absolutely convergent. Moreover from its very definition of $J(\varphi: x)$, it is left $H_{\mathbf{R}}$-invariant and right $K$-invariant as a function of $x \in G_{\mathbf{R}}$. From these remarks combined with the decomposition $G_{\mathbf{R}}=H_{\mathbf{R}} A_{\mathfrak{p}, \mathfrak{q}}^{+} K$, we see that the integral $J(\varphi: x)$ converges absolutely for all $x \in G_{\mathbf{R}}$. Now for any $X \in U\left(\mathfrak{g}_{\mathbf{C}}\right)$ the function $\varphi * X$ has the same property as $\varphi$, hence the integral $J(|\varphi * X|: x)$ is also finite. Therefore we have that the function $x \mapsto J(\varphi: x)$ is differentiable infinitely and we can exchange the order of differentiation and integration to have the formula

$$
J(\varphi: g) * X_{g}=J(\varphi * X: g), \quad X \in U\left(\mathfrak{g}_{\mathbf{C}}\right), g \in G_{\mathbf{R}} .
$$

Apply this formula to the Casimir element $\Omega$. Then Proposition 2.1.1 implies

$$
J\left(\varphi * \Omega: \exp \left(t Y_{0}\right)\right)=\gamma_{\mathfrak{h} \backslash \mathfrak{g}}(t)^{-1} \frac{d}{d t}\left(\gamma_{\mathfrak{h} \backslash \mathfrak{g}}(t) \frac{d}{d t} J\left(\varphi: \exp \left(t Y_{0}\right)\right)\right) .
$$

Hence

$$
\begin{aligned}
& \int_{\Gamma \backslash G_{\mathbf{R}}} G_{s}(x)\left(\varphi *\left(\Omega+\rho_{0}^{2}-s^{2}\right)\right)(x) d \dot{x}=\lim _{\eta \uparrow+\infty} \lim _{\zeta \downarrow 0} \\
& \times \int_{\zeta}^{\eta} \phi_{s}^{(2)}\left(a_{t}\right)\left\{\frac{d}{d t}\left(\gamma_{\mathfrak{h} \backslash \mathfrak{g}}(t) \frac{d}{d t} g(t)\right)+\left(\rho_{0}^{2}-s^{2}\right) \gamma_{\mathfrak{h} \backslash \mathfrak{g}}(t) g(t)\right\} d t
\end{aligned}
$$

where we put

$$
\begin{array}{rlrl}
a_{t} & =\exp \left(t Y_{0}\right), & & t>0, \\
g(t) & =J\left(\varphi: a_{t}\right), & t>0 .
\end{array}
$$


For any $\eta>\zeta>0$, integration by parts yields

$$
\begin{aligned}
& \int_{\zeta}^{\eta} \phi_{s}^{(2)}\left(a_{t}\right)\left\{\frac{d}{d t}\left(\gamma_{\mathfrak{h} \backslash \mathfrak{g}}(t) \frac{d}{d t} g(t)\right)+\left(\rho_{0}^{2}-s^{2}\right) \gamma_{\mathfrak{h} \backslash \mathfrak{g}}(t) g(t)\right\} d t \\
& =\gamma_{\mathfrak{h} \backslash \mathfrak{g}}(\eta) \phi_{s}^{(2)}\left(a_{\eta}\right) g^{\prime}(\eta)-\gamma_{\mathfrak{h} \backslash \mathfrak{g}}(\eta) g(\eta)\left(\left.\frac{d}{d t}\right|_{t=\eta} \phi_{s}^{(2)}\left(a_{t}\right)\right) \\
& \quad-\gamma_{\mathfrak{h} \backslash \mathfrak{g}}(\zeta) \phi_{s}^{(2)}\left(a_{\zeta}\right) g^{\prime}(\zeta)+\gamma_{\mathfrak{h} \backslash \mathfrak{g}}(\zeta) g(\zeta)\left(\left.\frac{d}{d t}\right|_{t=\zeta} \phi_{s}^{(2)}\left(a_{t}\right)\right) \\
& \quad-\int_{\zeta}^{\eta}\left\{\gamma_{\mathfrak{h} \backslash \mathfrak{g}}(t)^{-1} \frac{d}{d t}\left(\gamma_{\mathfrak{h} \backslash \mathfrak{g}}(t) \frac{d}{d t} \phi_{s}^{(2)}\left(a_{t}\right)\right)+\left(\rho_{0}^{2}-s^{2}\right) \phi_{s}^{(2)}\left(a_{t}\right)\right\} \\
& \quad \times \gamma_{\mathfrak{h} \backslash \mathfrak{g}}(t) g(t) d t .
\end{aligned}
$$

Since $\phi_{s}^{(2)}\left(a_{t}\right)$ is a solution of the differential equation $(\star)$, the integrand of the last term in the right-hand side of (3.2.5) is zero.

Now we have to evaluate the remaining four terms in the right-hand side of (3.2.5). To begin with we want to show

$$
\lim _{\eta \uparrow+\infty}\left\{\gamma_{\mathfrak{h} \backslash \mathfrak{g}}(\eta) \phi_{s}^{(2)}\left(a_{\eta}\right) g^{\prime}(\eta)-\gamma_{\mathfrak{h} \backslash \mathfrak{g}}(\eta) g(\eta)\left(\left.\frac{d}{d t}\right|_{t=\eta} \phi_{s}^{(2)}\left(a_{t}\right)\right)\right\}=0 .
$$

From (1.3.3), we have

$$
\gamma_{\mathfrak{h} \backslash \mathfrak{g}}(t) \prec e^{2 \rho_{0} t}, \quad\left|\gamma_{\mathfrak{h} \backslash \mathfrak{g}}^{\prime}(t)\right| \prec e^{2 \rho_{0} t}
$$

on $t>0$. Corollary 2.4.1 implies

$$
\left|\phi_{s}^{(2)}\left(a_{t}\right)\right| \prec e^{-\left(\operatorname{Re}(s)+\rho_{0}\right) t}, \quad\left|\frac{d}{d t} \phi_{s}^{(2)}\left(a_{t}\right)\right| \prec e^{-\left(\operatorname{Re}(s)+\rho_{0}\right) t}
$$

on $t>0$. Since

$$
g^{\prime}(t)=J\left(\varphi * Y_{0}: a_{t}\right), \quad t>0,
$$

we have the following estimates from (3.2.2):

$$
|g(t)| \prec e^{\tau \rho_{0} t}, \quad\left|g^{\prime}(t)\right| \prec e^{\tau \rho_{0} t}, \quad t>0 .
$$

Using (3.2.7), (3.2.8) and (3.2.9) with noting $2-\tau<\epsilon$, we have

$$
\begin{aligned}
\left|\phi_{s}^{(2)}\left(a_{\eta}\right) \gamma_{\mathfrak{h} \backslash \mathfrak{g}}(\eta) g^{\prime}(\eta)\right| & \prec e^{\left(-\operatorname{Re}(s)+(\tau+1) \rho_{0}\right) \eta}, \\
\left|\gamma_{\mathfrak{h} \backslash \mathfrak{g}}(\eta) g(\eta)\left(\left.\frac{d}{d t}\right|_{t=\eta} \phi_{s}^{(2)}\left(a_{t}\right)\right)\right| & \prec e^{\left(-\operatorname{Re}(s)+(\tau+1) \rho_{0}\right) \eta}
\end{aligned}
$$

on $t>0$. Since $\operatorname{Re}(s)>(\tau+1) \rho_{0}$, we finally obtain (3.2.6). 
Summing up the matters so far, the right-hand side of (3.2.5) is reduced to the sum

$$
-\gamma_{\mathfrak{h} \backslash \mathfrak{g}}(\zeta) \phi_{s}^{(2)}\left(a_{\zeta}\right) g^{\prime}(\zeta)+\gamma_{\mathfrak{h} \backslash \mathfrak{g}}(\zeta) g(\zeta)\left(\left.\frac{d}{d t}\right|_{t=\zeta} \phi_{s}^{(2)}\left(a_{t}\right)\right) .
$$

The first term in (3.2.10) vanishes if $\zeta \rightarrow 0$. In fact, from Corollary 2.4.1 and the estimate $\left|\gamma_{\mathfrak{h} \backslash \mathfrak{g}}(t)\right| \prec t$ near $t=0$, we have

$$
\left|\gamma_{\mathfrak{h} \backslash \mathfrak{g}}(\zeta) \phi_{s}^{(2)}\left(a_{\zeta}\right)\right| \prec \zeta|\log (\zeta)|
$$

that is valid for sufficiently small $\zeta>0$. Hence we have

$$
\lim _{\zeta \downarrow 0} \gamma_{\mathfrak{h} \backslash \mathfrak{g}}(\zeta) \phi_{s}^{(2)}\left(a_{\zeta}\right) g^{\prime}(\zeta)=J\left(\varphi * Y_{0}: e\right) \lim _{\zeta \downarrow 0}\left|\gamma_{\mathfrak{h} \backslash \mathfrak{g}}(\zeta) \phi_{s}^{(2)}\left(a_{\zeta}\right)\right|=0 .
$$

The remaining non-zero contribution to the right-hand side of (3.2.5) is only the fourth term. By differentiating the second formula in Corollary 2.4.1, we have

$$
\frac{d}{d t} \phi_{s}^{(2)}\left(a_{t}\right)=\frac{1}{t}+\log (t)+O(1)
$$

when $t>0$ is sufficiently small. Hence noting $\gamma_{\mathfrak{h} \backslash \mathfrak{g}}(t)=t(1+O(t)), t \downarrow 0$, we have

$$
\lim _{\zeta \downarrow 0}\left\{\gamma_{\mathfrak{h} \backslash \mathfrak{g}}(\zeta) g(\zeta)\left(\left.\frac{d}{d t}\right|_{t=\zeta} \phi_{s}^{(2)}\left(a_{t}\right)\right)\right\}=g(0) .
$$

From (3.2.4), (3.2.5), (3.2.6), (3.2.11) and (3.2.12), we finally have

$$
\int_{\Gamma \backslash G_{\mathbf{R}}} G_{s}(x)\left(\varphi *\left(\Omega+\rho_{0}^{2}-s^{2}\right)\right)(x) d \dot{x}=g(0) .
$$

Since

$$
g(0)=J(\varphi: e)=\int_{\Gamma_{H} \backslash H_{\mathbf{R}}} \int_{K} \varphi(h k) d k d \dot{h},
$$

the proof of (3) is now finished.

The map

$$
j: \Gamma_{H} \backslash H_{\mathbf{R}} \rightarrow \Gamma \backslash G_{\mathbf{R}}
$$

defined by

$$
j\left(\Gamma_{H} h\right)=\Gamma h, \quad h \in H_{\mathbf{R}}
$$

is a $C^{\infty}$-immersion and its image $C_{o}$ is closed in $\Gamma \backslash G_{\mathbf{R}}$ from the finiteness of the volume of $\Gamma_{H} \backslash H_{\mathbf{R}}$. Consider for simplicity the case when $\Gamma$ is neat so that 
its action on $G_{\mathbf{R}} / K$ is fixed points free; then the double coset space $\Gamma \backslash G_{\mathbf{R}} / K$ is naturally a manifold. The image $D_{o}$ of $C_{o}$ by the quotient map $\Gamma \backslash G_{\mathbf{R}} \rightarrow$ $\Gamma \backslash G_{\mathbf{R}} / K$ is a closed subset such that the natural map $\Gamma_{H} \backslash H_{\mathbf{R}} / K_{H} \rightarrow D_{o}$ is a finite covering; our basic assumption (see Proposition 2.4.1 (2)) implies that the real codimension of $D_{o}$ in $\Gamma \backslash G_{\mathbf{R}} / K$ is two.

Let $j_{*}(d \dot{h})$ be the image of $d \dot{h}$; it is a positive measure on $\Gamma \backslash G_{\mathbf{R}}$ such that

$$
\left\langle j_{*}(d \dot{h}), \varphi\right\rangle=\int_{\Gamma_{H} \backslash H_{\mathbf{R}}} \varphi(h) d \dot{h}, \quad \varphi \in C_{\mathrm{c}}\left(\Gamma \backslash G_{\mathbf{R}}\right) .
$$

Now we introduce a distribution $\delta_{D_{o}}$ by

$$
\begin{aligned}
\left\langle\delta_{D_{o}}, \varphi\right\rangle & =\left\langle j_{*}(d \dot{h})(x), \int_{K} \varphi(x k) d k\right\rangle \\
& =\int_{\Gamma_{H} \backslash H_{\mathbf{R}}} \int_{K} \varphi(h k) d k d \dot{h}, \quad \varphi \in C_{\mathrm{c}}^{\infty}\left(\Gamma \backslash G_{\mathbf{R}}\right) .
\end{aligned}
$$

Corollary 3.2.1. For $s \in \mathbf{C}$ with $\operatorname{Re}(s)>\rho_{0}$, the distribution $G_{s}$ satisfies the differential equation

$$
G_{s} *\left(\Omega+\rho_{0}^{2}-s^{2}\right)=\delta_{D_{o}} .
$$

Remark 3.2.1. Since $G_{s}$ is anihilated by an elliptic differential operator on the open set $Y=\Gamma \backslash G_{\mathbf{R}} / K-D_{o}$, the elliptic regularity theorem implies that $G_{s}$ is smooth on $Y$.

\section{§3.3. Coupling with $L^{2}$-automorphic forms}

As an application of Theorem 3.2.1, we compute the coupling of $G_{s}$ and square integrable automorphic forms on $\Gamma \backslash G_{\mathbf{R}}$. Note that, in Proposition 3.1.2, we have already done this when the coupled automorphic form is the constant function 1 .

Lemma 3.3.1. $\quad$ Let $\mathcal{S}$ be a Siegel domain for $\Gamma \backslash G_{\mathbf{R}}$ such that $G_{\mathbf{R}}=$ $\Gamma C \mathcal{S}$ with $C$ a finite subset of $G_{\mathbf{Q}}$. Let $\varphi \in L^{2}\left(\Gamma \backslash G_{\mathbf{R}}\right)$ be a $K$-invariant automorphic form satisfying

$$
\varphi * \Omega=\lambda_{\varphi} \varphi
$$

with $\lambda_{\varphi} \in \mathbf{C}$. Then there exists a positive number $\epsilon$ such that for any $X \in$ $U\left(\mathfrak{g}_{\mathbf{C}}\right)$ the evaluation

$$
|(\varphi * X)(\kappa g)| \prec a_{G}(g)^{(1-\epsilon) \rho_{G}}, \quad \kappa \in C, g \in \mathcal{S}
$$

holds. 
Proof. Let $C=\left\{\kappa_{1}, \ldots, \kappa_{r}\right\}$. Let $\Delta_{G}$ be the set of simple roots for $\left(A_{G}, P_{G}\right)$ and $\left\{Y_{\alpha}\right\}$ the dual basis of $\Delta_{G}$. Then, from [36, Theorem 4.4], for each $\kappa_{i} \in C$, there exists a linear form $\nu_{i} \in \mathfrak{a}_{G}^{*}=X_{\mathbf{Q}}\left(A_{G}\right) \otimes \mathbf{Z} \mathbf{R}$ with $\left\langle Y_{\alpha}, \nu_{i}\right\rangle>$ $0, \alpha \in \Delta_{G}$ such that

$$
\left|(\varphi * X)\left(\kappa_{i} g\right)\right| \prec a_{G}(g)^{\rho_{G}-\nu_{i}}, \quad g \in \mathcal{S} .
$$

Since $\left\langle Y_{\alpha}, \nu_{i}\right\rangle>0$ for $\alpha \in \Delta_{G}$, we can take a positive $\epsilon$ so that $\nu_{i}-\epsilon \rho_{G} \in{ }^{+} \mathfrak{a}_{G}^{*}$ for all $1 \leqslant i \leqslant r$, where ${ }^{+} \mathfrak{a}_{G}^{*}$ is the positive closed cone in $\mathfrak{a}_{G}^{*}$ generated by $\Delta_{G}$. Since

$$
a_{G}(g)^{\rho_{G}-\nu_{i}} \prec a_{G}(g)^{(1-\epsilon) \rho_{G}}, \quad g \in \mathcal{S}, 1 \leqslant i \leqslant r,
$$

we have done.

Proposition 3.3.1. We assume $\tau=\tau_{\mathbf{Q}}(G, \sigma)>1$. Let $\varphi \in L^{2}\left(\Gamma \backslash G_{\mathbf{R}}\right)$ be a $K$-invariant automorphic form belonging to the $\lambda_{\varphi}$-eigenspace of $\Omega$ with $\lambda_{\varphi} \in \mathbf{C}$. Let $s \in \mathbf{C}$ with $\operatorname{Re}(s)>(\tau+1) \rho_{0}$. Then we have

$$
\int_{\Gamma \backslash G_{\mathbf{R}}} G_{s}(x) \varphi(x) d \dot{x}=\frac{1}{\lambda_{\varphi}+\rho_{0}^{2}-s^{2}} \int_{\Gamma_{H} \backslash H_{\mathbf{R}}} \varphi(h) d \dot{h},
$$

where the integrals in the formula above are absolutely convergent.

Proof. By Lemma 3.3.1, the function $\varphi$ satisfies the assumption in Theorem 3.2.1. Hence applying the theorem, we obtain

$$
\int_{\Gamma \backslash G_{\mathbf{R}}} G_{s}(x)\left(\varphi *\left(\Omega+\rho_{0}^{2}-s^{2}\right)\right)(x) d \dot{x}=\int_{\Gamma_{H} \backslash H_{\mathbf{R}}} \varphi(h) d \dot{h} .
$$

Since $\varphi *\left(\Omega+\rho_{0}^{2}-s^{2}\right)=\left(\lambda_{\varphi}+\rho_{0}^{2}-s^{2}\right) \varphi$, we get the desired formula.

\section{$\S 4$. Estimate of Counting Function}

The purpose of this section is to establish an estimate of a function that counts the number of cosets in $\Gamma_{H} \backslash \Gamma$ lying on a certain bounded subset of $H_{\mathbf{R}} \backslash G_{\mathbf{R}}$; that estimate is used in the next section to have an $L^{p}$-estimate of the Poincaré series introduced in the previous section.

\section{§4.1. Finite dimensional $H_{\mathrm{R}}^{\circ}$-spherical representation}

In $\mathfrak{g}_{\mathbf{C}}$, we consider $\mathbf{R}$-subspaces

$$
\mathfrak{k}^{\mathrm{d}}=(\mathfrak{k} \cap \mathfrak{h})+\sqrt{-1}(\mathfrak{p} \cap \mathfrak{h}), \quad \mathfrak{p}^{\mathrm{d}}=(\mathfrak{p} \cap \mathfrak{q})+\sqrt{-1}(\mathfrak{k} \cap \mathfrak{q})
$$


and $\mathfrak{g}^{\mathrm{d}}=\mathfrak{k}^{\mathrm{d}}+\mathfrak{p}^{\mathrm{d}}$. Then $\mathfrak{g}^{\mathrm{d}}, \mathfrak{k}^{\mathrm{d}}$ and $\mathfrak{p}^{\mathrm{d}}$ are real forms of $\mathfrak{g}, \mathfrak{h}$ and $\mathfrak{q}$ respectively. Furthermore $\mathfrak{g}^{\mathrm{d}}$ and $\mathfrak{k}^{\mathrm{d}}$ are real Lie subalgebras of $\mathfrak{g}_{\mathbf{C}}$, which form a Riemannian symmetric pair $\left(\mathfrak{g}^{\mathrm{d}}, \mathfrak{k}^{\mathrm{d}}\right)$, the dual of $(\mathfrak{g}, \mathfrak{h})$. Let $\mathfrak{a}^{\mathrm{d}}$ be a maximal abelian subspace of $\mathfrak{p}^{\mathrm{d}}$ extending $\mathfrak{a}_{\mathfrak{p}, \mathfrak{q}}=\mathbf{R} Y_{0}$ and $\Phi^{\mathrm{d}}$ the restricted root system of $\mathfrak{g}^{\mathrm{d}}$ with respect to $\mathfrak{a}^{\mathrm{d}}$. Fix a positive system $\Phi_{+}^{\mathrm{d}}$ of $\Phi^{\mathrm{d}}$ such that $\beta\left(Y_{0}\right) \geqslant 0$ for all $\beta \in \Delta^{\mathrm{d}}$ with $\Delta^{\mathrm{d}}$ the set of simple roots.

Proposition 4.1.1. Let $\mathcal{L}^{+}\left(\mathfrak{a}^{\mathrm{d}}\right)$ be the set of all $\mu \in\left(\mathfrak{a}^{\mathrm{d}}\right)^{*}$ that satisfy the condition

$$
\frac{\langle\mu, \beta\rangle}{\langle\beta, \beta\rangle} \in \mathbf{N}, \quad \beta \in \Phi_{+}^{\mathrm{d}} .
$$

For each $\mu \in \mathcal{L}^{+}\left(\mathfrak{a}^{\mathrm{d}}\right)$, there corresponds an irreducible representation $\left(\sigma_{\mu}^{\mathrm{d}}, V_{\mu}\right)$ of $\mathfrak{g}^{\mathrm{d}}$ that has the two properties:

(a) The vectors $v \in V_{\mu}$ such that $\sigma_{\mu}^{\mathrm{d}}(X) v=0$ for all $X \in \mathfrak{k}^{\mathrm{d}}$ form a one dimensional subspace.

(b) The $\mu$ is a highest restricted weight in $V_{\mu}$, that is if $\nu \in\left(\mathfrak{a}^{\mathrm{d}}\right)^{*}$ occurs in $V_{\mu}$ as a weight of $\mathfrak{a}^{\mathrm{d}}$ then there exist non negative integers $a_{\beta}$ such that $\nu=\mu-\sum_{\beta \in \Delta_{\mathrm{d}}} a_{\beta} \cdot \beta$. Furthermore the $\mu$-weight space is one dimensional.

Proof. See [18, IX, Section 5].

Let $\mu \in \mathcal{L}^{+}\left(\mathfrak{a}^{\mathrm{d}}\right)$. Then the representation $\sigma_{\mu}^{\mathrm{d}}$ gives rise to an irreducible representation of $\mathfrak{g}_{\mathbf{C}}$, say $\sigma_{\mu}^{\mathbf{C}}$, through the complexification. Let $\left(\sigma_{\mu}, V_{\mu}\right)$ be the representation of $\mathfrak{g}$ obtained by restriction of $\sigma_{\mu}^{\mathbf{C}}$ to $\mathfrak{g}$. Since $\mathfrak{h}$ is a real form of $\mathfrak{k}^{\mathrm{d}}$, any $\mathfrak{k}^{\mathrm{d}}$-fixed vector of $\sigma_{\mu}^{\mathrm{d}}$ is automatically fixed by $\mathfrak{h}$ acted upon $V_{\mu}$ through $\sigma_{\mu}$.

To obtain an $H_{\mathbf{R}^{\circ}}^{\circ}$-spherical representation of $G_{\mathbf{R}}$, we may proceed as follows: For $\mu \in \mathcal{L}^{+}\left(\mathfrak{a}^{\mathrm{d}}\right)$, if we take a positive integer $d$ suitably, then the representation $\sigma_{d \mu}$ can be integrated to $G_{\mathbf{R}}^{\circ}$ to give rise to a representation of that group. Since we have assumed $G_{\mathbf{R}}$ is connected, we have an irreducible finite dimensional representation of $G_{\mathbf{R}}$ whose underlying $\mathfrak{g}$-module is $\left(\sigma_{d \mu}, V_{d \mu}\right)$. Let $v$ be a $\mathfrak{h}$-fixed vector in $V_{d \mu}$; then it is also fixed by the action of $H_{\mathbf{R}}^{\circ}$.

Lemma 4.1.1. Let $\left(\sigma_{\mu}, V_{\mu}\right)$ with $\mu \in \mathcal{L}^{+}\left(\mathfrak{a}^{\mathrm{d}}\right)$ be an irreducible finite dimensional $H_{\mathbf{R}}^{\circ}$-spherical representation of $G_{\mathbf{R}}$. Put $\mu_{0}=\mu\left(Y_{0}\right)$. Then there exists a basis of $V_{\mu}$ with respect to which $\sigma_{\mu}\left(Y_{0}\right)$ is represented by a diagonal matrix. Let $S_{\mu}$ be the set of eigenvalues of $\sigma_{\mu}\left(Y_{0}\right)$; then $S_{\mu}$ is a finite subset of $\mathbf{R}$ such that $\sup S_{\mu}=\mu_{0}$ and inf $S_{\mu}=-\mu_{0}$. 
Proof. Let $\Pi$ be the set of $\mathfrak{a}^{\mathrm{d}}$-weights occuring in $V_{\mu}$ and $\left\{v_{i} \mid 1 \leqslant i \leqslant d_{\mu}\right\}$ a basis of $V_{\mu}$ consisting of $\mathfrak{a}^{\mathrm{d}}$-weight vectors. Since $Y_{0} \in \mathfrak{a}^{\mathrm{d}}$, the operator $\sigma_{\mu}\left(Y_{0}\right)$ is obviously represented by a diagonal matrix with respect to the basis $\left\{v_{i}\right\}$ and

$$
S_{\mu}=\left\{\nu\left(Y_{0}\right) \mid \nu \in \Pi\right\} .
$$

By Proposition 4.1.1, any weight $\nu \in \Pi$ is of the form $\mu-\sum_{\beta \in \Delta^{\mathrm{d}}} c_{\beta} \cdot \beta$ with $c_{\beta} \in \mathbf{N}$. Since $\beta\left(Y_{0}\right) \in \mathbf{N}$ for any $\beta \in \Delta^{\mathrm{d}}$, we have

$$
\nu\left(Y_{0}\right)=\mu_{0}-\sum_{\beta \in \Delta^{\mathrm{d}}} c_{\beta} \cdot \beta\left(Y_{0}\right) \leqslant \mu_{0} .
$$

Hence $\sup S_{\mu} \leqslant \mu_{0}$. Noting $\mu \in \Pi$ and $\mu\left(Y_{0}\right)=\mu_{0}$, we indeed have $\sup S_{\mu}=\mu_{0}$. To conclude the proof, it suffices to show $-S_{\mu}=S_{\mu}$. Since $H_{\mathbf{R}} \backslash G_{\mathbf{R}}$ is of split rank one, there exists an element $w_{0} \in N_{H_{\mathbf{R}} \cap K}\left(\mathfrak{a}_{\mathfrak{p}, \mathfrak{q}}\right)$ such that $\operatorname{Ad}\left(w_{0}\right)$ acts on $\mathfrak{a}_{\mathfrak{p}, \mathfrak{q}}$ by the scalar -1 . Then if $v \in V_{\mu}$ is a $\nu_{0}$-eigenvector of $\sigma_{\mu}\left(Y_{0}\right)$ then $\sigma_{\mu}\left(w_{0}\right) v$ is a $\left(-\nu_{0}\right)$-eigenvector of $\sigma_{\mu}\left(Y_{0}\right)$. Thus we have $-S_{\mu}=S_{\mu}$ as desired.

\section{$\S 4.2$. Gauge function}

Let $\left(\sigma_{\mu}, V_{\mu}\right)$ be an irreducible finite dimensional $H_{\mathbf{R}^{-}}^{\circ}$ spherical representation of $G_{\mathbf{R}}$ with $\mu \in \mathcal{L}^{+}\left(\mathfrak{a}^{\mathrm{d}}\right)$ such that $\mu_{0}:=\mu\left(Y_{0}\right)>0$. (We can take such a representation as we remarked in the previous subsecton.) Let (|) $: V_{\mu} \times V_{\mu} \rightarrow \mathbf{C}$ be a positive definite Hermitian inner product on $V_{\mu}$ such that

(a) For any $k \in K$, the operator $\sigma_{\mu}(k)$ is unitary with respect to $(\mid)$,

(b) For any $X \in \mathfrak{p}$, the operator $\sigma_{\mu}(X)$ is self-adjoint with respect to $(\mid)$.

Let $\|v\|=\sqrt{(v \mid v)}$ be the associated norm on $V_{\mu}$.

Definition 4.2.1. The function $\varphi_{\mu}: G_{\mathbf{R}} \rightarrow \mathbf{R}_{+}$defined by

$$
\varphi_{\mu}(g)=\left\|\sigma_{\mu}\left(g^{-1}\right) v_{H}\right\|, \quad g \in G_{\mathbf{R}}
$$

will be called the gauge function associated with $\sigma_{\mu}$ (or $\mu$ ), where $v_{H}$ is an $H_{\mathbf{R}}^{\circ}$-fixed unit vector in $V_{\mu}$.

From definition we clearly have that the function $\varphi_{\mu}$ is left $H_{\mathbf{R}}^{\circ}$-invariant and right $K$-invariant.

Lemma 4.2.1. $\quad$ The gauge function $\varphi_{\mu}$ associated with $\mu$ is left $H_{\mathbf{R}}$-invariant. 
Proof. Since $H_{\mathbf{R}}^{\circ}$ is a normal subgroup of $H_{\mathbf{R}}$ with finite index, $H_{\mathbf{R}}$ acts on the one dimensional space $V^{H_{\mathbf{R}}^{\circ}}=V^{\mathfrak{k}^{\mathrm{d}}}$ by a unitary character. From this remark, our lemma follows.

Proposition 4.2.1. Put $\mu_{0}=\mu\left(Y_{0}\right)$. Then for any $s \in \mathbf{R}$ with $s>$ $2 \rho_{0} \mu_{0}^{-1}$, we have

$$
\int_{0}^{\infty} \varphi_{\mu}\left(\exp \left(t Y_{0}\right)\right)^{-s} \gamma_{\mathfrak{h} \backslash \mathfrak{g}}(t) d t<+\infty .
$$

Proof. Let $S_{\mu}$ be as in Lemma 4.1.1. For any $\nu \in S_{\mu}$ let $V_{\mu}^{[\nu]}$ be the $\nu$-eigenspace of $\sigma_{\mu}\left(Y_{0}\right)$ in $V_{\mu}$. Since $V_{\mu}$ is a direct sum of spaces $V_{\mu}^{[\nu]}$ 's, we can write $v_{H}$ as

$$
v_{H}=\sum_{\nu} v^{(\nu)}, \quad v^{(\nu)} \in V_{\mu}^{[\nu]} .
$$

Since $\sigma_{\mu}\left(Y_{0}\right)$ is self-adjoint, two eigenvectors of $\sigma_{\mu}\left(Y_{0}\right)$ that belong to different eigenvalues are orthogonal to one another. Hence we have

$$
\begin{aligned}
\varphi_{\mu}\left(\exp \left(t Y_{0}\right)\right) & =\left\|\sigma_{\mu}\left(\exp \left(t Y_{0}\right)\right)^{-1} v_{H}\right\| \\
& =\left\|\sum_{\nu} e^{-t \nu} v^{(\nu)}\right\| \\
& =\left(\sum_{\nu} e^{-2 t \nu}\left\|v^{(\nu)}\right\|^{2}\right)^{1 / 2} .
\end{aligned}
$$

Since $\nu \geqslant-\mu_{0}$ if $v^{(\nu)} \neq 0$, we have

$$
\lim _{t \rightarrow+\infty} \frac{\varphi_{\mu}\left(\exp \left(t Y_{0}\right)\right)}{e^{t \mu_{0}}}=\lim _{t \rightarrow+\infty}\left(\sum_{\nu} e^{-2 t\left(\nu+\mu_{0}\right)}\left\|v^{(\nu)}\right\|^{2}\right)^{1 / 2}=\left\|v^{\left(-\mu_{0}\right)}\right\| .
$$

We show that $v^{\left(-\mu_{0}\right)} \neq 0$. Assume contrary that $v^{\left(-\mu_{0}\right)}=0$, or equivalently $v_{H}$ is orthogonal to $V_{\mu}^{\left[-\mu_{0}\right]}$. Put $\overline{\mathfrak{u}}=\mathfrak{g}_{-\lambda}+\mathfrak{g}_{-2 \lambda}$. Since $V_{\mu}^{[\nu]}=\{0\}$ for $|\nu|>\mu_{0}$ and since $\mathfrak{g}_{-j \lambda} V_{\mu}^{[i]} \subset V_{\mu}^{[i-j]}$ for $j=0,1,2$, we have $\sigma_{\mu}(\overline{\mathfrak{u}}) V_{\mu}^{\left[-\mu_{0}\right]}=\{0\}$ and $\sigma_{\mu}\left(\mathfrak{g}_{0}\right) V_{\mu}^{\left[-\mu_{0}\right]} \subset V_{\mu}^{\left[-\mu_{0}\right]}$. Noting the decomposition $\mathfrak{g}=\mathfrak{g}_{0}+\overline{\mathfrak{u}}+\mathfrak{h}$, we have $\sigma_{\mu}\left(U\left(\mathfrak{h}_{\mathbf{C}}\right)\right) V_{\mu}^{\left[-\mu_{0}\right]}=\sigma_{\mu}\left(U\left(\mathfrak{g}_{\mathbf{C}}\right)\right) V_{\mu}^{\left[-\mu_{0}\right]}$, hence $\sigma_{\mu}\left(U\left(\mathfrak{h}_{\mathbf{C}}\right)\right) V_{\mu}^{\left[-\mu_{0}\right]}=V_{\mu}$ because $\sigma_{\mu}$ is irreducible and $V_{\mu}^{\left[-\mu_{0}\right]} \neq\{0\}$. For any $X \in \mathfrak{h}$, we can write it as $X=$ $X^{+}+X^{-}$with $X^{+} \in \mathfrak{k} \cap \mathfrak{h}$ and $X^{-} \in \mathfrak{p} \cap \mathfrak{h}$. Since $\sigma_{\mu}\left(X^{ \pm}\right) v_{H}=0$, we have

$$
\begin{aligned}
\left(\sigma_{\mu}(X) v \mid v_{H}\right) & =\left(\sigma_{\mu}\left(X^{+}\right) v \mid v_{H}\right)+\left(\sigma_{\mu}\left(X^{-}\right) v \mid v_{H}\right) \\
& =-\left(v \mid \sigma_{\mu}\left(X^{+}\right) v_{H}\right)+\left(v \mid \sigma_{\mu}\left(X^{-}\right) v_{H}\right) \\
& =0
\end{aligned}
$$


for any $v \in V_{\mu}$. This shows that $v_{H}$ is orthogonal to $\sigma_{\mu}(D) V_{\mu}^{\left[-\mu_{0}\right]}$ for any $D \in U\left(\mathfrak{h}_{\mathbf{C}}\right)$ without the constant term. Since $v_{H}$ is assumed to be orthogonal to $V_{\mu}^{\left[-\mu_{0}\right]}$, we have that $v_{H}$ is orthogonal to $\sigma_{\mu}\left(U\left(\mathfrak{h}_{\mathbf{C}}\right)\right) V_{\mu}^{\left[-\mu_{0}\right]}=V_{\mu}$; hence $v_{H}=0$, a contradiction.

By (4.2.1), there exists a $t_{0}>0$ such that

$$
\left|\frac{\varphi_{\mu}\left(\exp \left(t Y_{0}\right)\right)}{e^{t \mu_{0}}}-\left\|v^{\left(-\mu_{0}\right)}\right\|\right| \leqslant \frac{1}{2}\left\|v^{\left(-\mu_{0}\right)}\right\|
$$

for all $t>t_{0}$. Hence we have

$$
\varphi_{\mu}\left(\exp \left(t Y_{0}\right)\right) \geqslant \frac{1}{2}\left\|v^{\left(-\mu_{0}\right)}\right\| e^{t \mu_{0}}, \quad t>t_{0} .
$$

and

$$
\varphi_{\mu}\left(\exp \left(t Y_{0}\right)\right)^{-s} \leqslant\left(\frac{1}{2}\left\|v^{\left(-\mu_{0}\right)}\right\|\right)^{-s} e^{-s t \mu_{0}}, \quad t>t_{0}, s>0 .
$$

Noting that $\gamma_{\mathfrak{h} \backslash \mathfrak{g}}(t) \prec e^{2 t \rho_{0}}, t>t_{0}$, we have the following estimate to conclude the proof:

$$
\int_{t_{0}}^{\infty} \varphi_{\mu}\left(\exp \left(t Y_{0}\right)\right)^{-s} \gamma_{\mathfrak{h} \backslash \mathfrak{g}}(t) d t \prec \int_{t_{0}}^{\infty} e^{-\left(s \mu_{0}-2 \rho_{0}\right) t} d t<+\infty
$$

if $s>2 \rho_{0} \mu_{0}^{-1}$.

Corollary 4.2.1. If $s>2 \rho_{0} \mu_{0}^{-1}$, then we have

$$
\int_{\Gamma_{H} \backslash G_{\mathbf{R}}} \varphi_{\mu}(g)^{-s} d \dot{g}<+\infty .
$$

Proof. Indeed, using (1.3.2), we have

$$
\begin{aligned}
& \int_{\Gamma_{H} \backslash G_{\mathbf{R}}} \varphi_{\mu}(g)^{-s} d \dot{g} \\
& \quad=\int_{\Gamma_{H} \backslash H_{\mathbf{R}}} d \dot{h} \int_{K} d k \int_{0}^{\infty} \varphi_{\mu}\left(\exp \left(t Y_{0}\right)\right)^{-s} \gamma_{\mathfrak{h} \backslash \mathfrak{g}}(t) d t \\
& \quad=\operatorname{vol}\left(\Gamma_{H} \backslash H_{\mathbf{R}}\right) \int_{0}^{\infty} \varphi_{\mu}\left(\exp \left(t Y_{0}\right)\right)^{-s} \gamma_{\mathfrak{h} \backslash \mathfrak{g}}(t) d t \\
& \quad<+\infty
\end{aligned}
$$




\section{§4.3. Counting function and its estimate}

Let $\Gamma$ be an arithmetic subgroup of $G_{\mathbf{Q}}$. Taking the gauge function $\varphi_{\mu}$ associated with $\mu \in \mathcal{L}^{+}\left(\mathfrak{a}^{\mathrm{d}}\right)$, we put

$$
D_{\mu}(r)=\left\{\dot{x} \in \Gamma_{H} \backslash G_{\mathbf{R}} \mid \varphi_{\mu}(x) \leqslant r\right\}, \quad r>0 .
$$

Now we introduce a function $N_{\mu}(r: \dot{g})$ to count the number of cosets in $\Gamma_{H} \backslash \Gamma$ lying on the set $D_{\mu}(r)$. We have to controle its behavior when $g$ varies on a Siegel set of $G_{\mathbf{R}}$ and $r$ goes to infinity.

Definition 4.3.1. For $\dot{g} \in \Gamma \backslash G_{\mathbf{R}}$ and $r>0$, we put

$$
N_{\mu}(r: \dot{g})=\operatorname{Card}\left\{\gamma \in \Gamma_{H} \backslash \Gamma \mid \varphi_{\mu}(\gamma g) \leqslant r\right\} .
$$

We prepare lemmas for later use.

Lemma 4.3.1. Let $\sigma_{\mu}^{*}$ be the contragradient representation of $\sigma_{\mu}$. Then we have the inequality

$$
\varphi_{\mu}(x y) \leqslant d_{\mu}^{1 / 2}\left\|\sigma_{\mu}^{*}(y)\right\|_{\mathrm{op}} \varphi_{\mu}(x), \quad x, y \in G_{\mathbf{R}},
$$

where $\left\|\sigma_{\mu}^{*}(y)\right\|_{\text {op }}$ means the operator norm of $\sigma_{\mu}^{*}(y)$ and $d_{\mu}=\operatorname{dim}_{\mathbf{C}} V_{\mu}$.

Proof. Let $\left\{v_{i}\right\}$ be an orthonormal basis of $V_{\mu}$ and $\left\{v_{i}^{*}\right\}$ its dual basis. Then

$$
\sigma_{\mu}(x)^{-1} v_{H}=\sum_{i}\left\langle\sigma_{\mu}(x)^{-1} v_{H}, v_{i}^{*}\right\rangle v_{i}, \quad x \in G_{\mathbf{R}}
$$

Hence

$$
\begin{aligned}
\varphi_{\mu}(x)=\left\|\sigma_{\mu}(x)^{-1} v_{H}\right\| & =\left(\sum_{i}\left|\left\langle\sigma_{\mu}\left(x^{-1}\right) v_{H}, v_{i}^{*}\right\rangle\right|^{2}\right)^{1 / 2} \\
& \leqslant d_{\mu}^{1 / 2} \sup _{i}\left|\left\langle\sigma_{\mu}\left(x^{-1}\right) v_{H}, v_{i}^{*}\right\rangle\right|
\end{aligned}
$$

Put

$$
\psi_{i}(x)=\left\langle\sigma_{\mu}\left(x^{-1}\right) v_{H}, v_{i}^{*}\right\rangle, \quad x \in G_{\mathbf{R}} .
$$

Then we have

$$
\varphi_{\mu}(x y) \leqslant d_{\mu}^{1 / 2} \sup _{i}\left|\psi_{i}(x y)\right|, \quad x, y \in G_{\mathbf{R}}
$$


and

$$
\begin{aligned}
\left|\psi_{i}(x y)\right| & =\left|\left\langle\sigma_{\mu}\left(y^{-1}\right) \sigma_{\mu}\left(x^{-1}\right) v_{H}, v_{i}^{*}\right\rangle\right| \\
& =\left|\left\langle\sigma_{\mu}\left(x^{-1}\right) v_{H}, \sigma_{\mu}^{*}(y) v_{i}^{*}\right\rangle\right| \\
& \leqslant\left\|\sigma_{\mu}\left(x^{-1}\right) v_{H}\right\| \cdot\left\|\sigma_{\mu}^{*}(y) v_{i}^{*}\right\| \\
& \leqslant\left\|\sigma_{\mu}^{*}(y)\right\|_{\text {op }} \varphi_{\mu}(x) .
\end{aligned}
$$

Lemma 4.3.2. Let $\mathcal{N}$ be a relatively compact neighborhood of the identity of $G_{\mathbf{R}}$ and $\chi_{\mathcal{N}}$ the characteristic function of $\mathcal{N}$ on $G_{\mathbf{R}}$. Put

$$
\xi_{\mathcal{N}}(x, g)=\sum_{\gamma \in \Gamma} \chi_{\mathcal{N}}\left(x^{-1} \gamma g\right), \quad x, g \in G_{\mathbf{R}}
$$

Then we have

(i) $\xi_{\mathcal{N}}(x, g)=0, \quad g \notin \Gamma x \mathcal{N}$.

(ii) For a Siegel set $\mathcal{S}$, we have

$$
\xi_{\mathcal{N}}(x, g) \prec a_{G}(x)^{2 \rho_{G}}, \quad x \in \mathcal{S}, g \in G_{\mathbf{R}} .
$$

Proof. (i) is obvious. The estimate in (ii) is proved in [23, pp. 59-60].

Lemma 4.3.3. Let $\mathcal{N}$ be a relatively compact neighborhood of the identity in $G_{\mathbf{R}}$. Put

$$
c_{\mu}(\mathcal{N})=d_{\mu}^{1 / 2} \sup _{y \in \mathcal{N}}\left\|\sigma_{\mu}^{*}(y)\right\|_{\text {op }}
$$

Let $\mathcal{S}$ be a Siegel set of $G_{\mathbf{R}}$. Then we have an estimate

$$
\sum_{\gamma \in \Gamma_{H} \backslash \Gamma} \varphi_{\mu}(\gamma x)^{-s} \prec c_{\mu}(\mathcal{N})^{s} a_{G}(x)^{2 \rho_{G}} \int_{\Gamma_{H} \backslash(\Gamma x \mathcal{N})} \varphi_{\mu}(g)^{-s} d \dot{g}, \quad x \in \mathcal{S}, s>0 .
$$

Proof. From Lemma 4.3.1 we have

$$
\varphi_{\mu}(\gamma x y) \leqslant c_{\mu}(\mathcal{N}) \varphi_{\mu}(\gamma x), \quad \gamma \in \Gamma, x \in G_{\mathbf{R}}, y \in \mathcal{N} .
$$

Hence if $s>0$, we have

$$
c_{\mu}(\mathcal{N})^{-s} \varphi_{\mu}(\gamma x)^{-s} \leqslant \varphi_{\mu}(\gamma x y)^{-s}, \quad \gamma \in \Gamma, x \in G_{\mathbf{R}}, y \in \mathcal{N} .
$$


Let $\chi_{\mathcal{N}}$ be the characteristic function of the set $\mathcal{N}$. By integrating with respect to $y \in \mathcal{N}$ and then taking the summation with respect to $\gamma \in \Gamma_{H} \backslash \Gamma$, from (4.3.2), we have

$$
\begin{aligned}
c_{\mu}(\mathcal{N})^{-s} \operatorname{vol}(\mathcal{N}) \sum_{\gamma \in \Gamma_{H} \backslash \Gamma} \varphi_{\mu}(\gamma x)^{-s} \\
\leqslant \sum_{\gamma \in \Gamma_{H} \backslash \Gamma} \int_{\mathcal{N}} \varphi_{\mu}(\gamma x y)^{-s} d y \\
=\sum_{\gamma \in \Gamma_{H} \backslash \Gamma} \int_{\gamma x \mathcal{N}} \varphi_{\mu}(g)^{-s} d g \\
=\sum_{\gamma \in \Gamma_{H} \backslash \Gamma} \int_{G_{\mathbf{R}}} \chi_{\mathcal{N}}\left(x^{-1} \gamma^{-1} g\right) \varphi_{\mu}(g)^{-s} d g \\
=\sum_{\gamma \in \Gamma_{H} \backslash \Gamma} \int_{\Gamma_{H} \backslash G_{\mathbf{R}}}\left(\sum_{\delta \in \Gamma_{H}} \chi_{\mathcal{N}}\left(x^{-1} \gamma^{-1} \delta g\right)\right) \varphi_{\mu}(g)^{-s} d \dot{g} \\
=\int_{\Gamma_{H} \backslash G_{\mathbf{R}}}\left(\sum_{\gamma \in \Gamma_{H} \backslash \Gamma} \sum_{\delta \in \Gamma_{H}} \chi_{\mathcal{N}}\left(x^{-1} \gamma^{-1} \delta g\right)\right) \varphi_{\mu}(g)^{-s} d \dot{g} \\
=\int_{\Gamma_{H} \backslash G_{\mathbf{R}}}\left(\sum_{\gamma \in \Gamma} \chi_{\mathcal{N}}\left(x^{-1} \gamma^{-1} g\right)\right) \varphi_{\mu}(g)^{-s} d \dot{g} \\
=\int_{\Gamma_{H} \backslash G_{\mathbf{R}}} \xi_{\mathcal{N}}(x, g) \varphi_{\mu}(g)^{-s} d \dot{g} .
\end{aligned}
$$

Using Lemma 4.3.2, we get the conclusion.

Corollary 4.3.1. Let $\mathcal{S}$ be a Siegel set in $G_{\mathbf{R}}$. Then we have

$$
\begin{gathered}
N_{\mu}(r: \dot{g}) \prec r^{s} c_{\mu}(\mathcal{N})^{s} a_{G}(g)^{2 \rho_{G}} \int_{\Gamma_{H} \backslash(\Gamma g \mathcal{N})} \varphi_{\mu}(x)^{-s} d \dot{x}, \\
\quad r>0, s>0, g \in \mathcal{S} .
\end{gathered}
$$

Proof. This follows from the previous lemma if one notes that

$$
r^{-s} N_{\mu}(r: \dot{g}) \leqslant \sum_{\gamma \in \Gamma_{H} \backslash \Gamma} \varphi_{\mu}(\gamma g)^{-s} .
$$

Corollary 4.3.2. For $r>0$ and $\dot{g} \in \Gamma \backslash G_{\mathbf{R}}$, the number $N_{\mu}(r: \dot{g})$ is finite. 
Proof. This is a consequence of Corollaries 4.3.1 and 4.2.1. Indeed, we may take $s$ with $s>2 \rho_{0} \mu_{0}^{-1}$ in the formula (4.3.4); then the right hand side of (4.3.4) is finite by Corollary 4.2.1.

Recall that $\mathcal{S}_{H}$, a Siegel domain for $\Gamma_{H} \backslash H_{\mathbf{R}}$, was divided into $m$ open subsets $\mathcal{S}_{H}^{(j)} ;$ Proposition 1.5.1.

Lemma 4.3.4. For any $t_{1}>0$ and $1 \leqslant j \leqslant m$, put

$$
\mathcal{S}_{H}^{(j)}\left[t_{1}\right]=\left\{h \in \mathcal{S}_{H}^{(j)} \mid a_{H}(h)^{\rho_{H}^{(j)}} \geqslant t_{1}\right\} .
$$

Let $\eta \geqslant 0$ be such that $2 \rho_{H}-\eta \rho_{H}^{(j)} \in{ }^{+} \mathfrak{a}_{H}^{*}$. Then we have

$$
\int_{\mathcal{S}_{H}^{(j)}\left[t_{1}\right]} d h \prec t_{1}^{-\eta}\left(1+\left|\log t_{1}\right|^{l}\right), \quad t_{1}>0,
$$

where $l$ is the semisimple $\mathbf{Q}-$ rank of $H$.

Proof. This follows from [33, Lemma 6.15].

Proposition 4.3.1. Let $\eta \geqslant 0$ be such that

$$
2 \rho_{H}-\eta \rho_{H}^{(j)} \in{ }^{+} \mathfrak{a}_{H}^{*}, \quad 1 \leqslant j \leqslant m .
$$

Let $\mathcal{N}$ be a relatively compact neighborhood of the identity of $G_{\mathbf{R}}$ and $s$ a real number with $s>(2+\eta) \rho_{0} \mu_{0}^{-1}$. Let $\mathcal{S}$ be a Siegel domain for $\Gamma \backslash G_{\mathbf{R}}$. Then we have an estimate

$$
\int_{\Gamma_{H} \backslash(\Gamma g \mathcal{N})} \varphi_{\mu}(x)^{-s} d \dot{x} \prec a_{G}(g)^{-\eta \rho_{G}}\left(1+\left|\log a_{G}(g)^{\rho_{G}}\right|^{l}\right), \quad g \in \mathcal{S},
$$

where $l$ is the semisimple $\mathbf{Q}-$ rank of $H$.

Proof. We follow the argument in [33, 6.18]. By Proposition 1.5.2, there exists a positive constant $c_{1}$ such that if

$$
\begin{aligned}
\gamma g u & =\delta \kappa_{H} h \exp \left(t Y_{0}\right) k, \\
\gamma & \in \Gamma, g \in \mathcal{S}, u \in \mathcal{N}, \\
\delta & \in \Gamma_{H}, \kappa_{H} \in C_{H}, h \in \mathcal{S}_{H}^{(j)}, t>0, k \in K,
\end{aligned}
$$

then $c_{1} \cdot e^{-t \rho_{0}} a_{H}(h)^{-\rho_{H}^{(j)}} \leqslant a_{G}(g)^{-\rho_{G}}$. Combining this with Proposition 1.5.1, we have

$$
\Gamma g \mathcal{N} \subset \bigcup_{j=1}^{m} \Gamma_{H} C_{H} \mathcal{S}_{H}^{(j)}\left[c_{1} \cdot e^{-t \rho_{0}} a_{G}(g)^{\rho_{G}}\right] A_{\mathfrak{p}, \mathfrak{q}}^{+} K, \quad g \in \mathcal{S},
$$


with the notation in Lemma 4.3.4. Hence we have

$$
\begin{aligned}
& \int_{\Gamma_{H} \backslash(\Gamma g \mathcal{N})} \varphi_{\mu}(x)^{-s} d \dot{x} \\
& \prec \sum_{j=1}^{m} \int_{\mathcal{S}_{H}^{(j)}\left[c_{1} \cdot e^{\left.-t \rho_{0} a_{G}(g)^{\rho} G\right]} A_{\mathfrak{p},{ }_{\mathfrak{q}} K}^{+}\right.} \varphi_{\mu}(x)^{-s} d x \\
& \prec \sum_{j=1}^{m} \int_{0}^{\infty} \varphi_{\mu}\left(\exp \left(t Y_{0}\right)\right)^{-s} \gamma_{\mathfrak{h} \backslash \mathfrak{g}}(t)\left(\int_{\mathcal{S}_{H}^{(j)}\left[c_{1} e^{-t \rho_{0} a_{G}}(g)^{\rho_{G}}\right]} d h\right) d t \\
& \prec \int_{0}^{\infty} \varphi_{\mu}\left(\exp \left(t Y_{0}\right)\right)^{-s} \gamma_{\mathfrak{h} \backslash \mathfrak{g}}(t) a_{G}(g)^{-\eta \rho_{G}} e^{t \eta \rho_{0}} \\
& \times\left(1+\left|\log \left(c_{1} \cdot a_{G}(g)^{\rho_{G}} e^{-t \rho_{0}}\right)\right|^{l}\right) d t .
\end{aligned}
$$

To have the last estimation above, we used Lemma 4.3.4. From (1.3.3) and the inequality (4.2.2), we have

$$
\gamma_{\mathfrak{h} \backslash \mathfrak{g}}(t) \prec e^{2 \rho_{0} t}, \quad\left|\varphi_{\mu}\left(\exp \left(t Y_{0}\right)\right)^{-s}\right| \prec e^{-s \mu_{0} t}
$$

for $t>0$. Using these estimates, from (4.3.5) we obtain

$$
\begin{aligned}
& \int_{\Gamma_{H} \backslash \Gamma g \mathcal{N}} \varphi_{\mu}(x)^{-s} d \dot{x} \\
& \quad \prec a_{G}(g)^{-\eta \rho_{G}} \int_{0}^{\infty} e^{\left((2+\eta) \rho_{0}-s \mu_{0}\right) t}\left(1+\left|\log \left(c_{1} \cdot a_{G}(g)^{\rho_{G}} e^{-t \rho_{0}}\right)\right|^{l}\right) d t \\
& \quad \prec a_{G}(g)^{-\eta \rho_{G}}\left(1+\left|\log a_{G}(g)^{\rho_{G}}\right|^{l}\right) \int_{0}^{\infty} e^{\left((2+\eta) \rho_{0}-s \mu_{0}\right) t} \\
& \quad \times\left(1+\left(\left|\log \left(c_{1}\right)\right|+t \rho_{0}\right)^{l}\right) d t .
\end{aligned}
$$

Since $s>(2+\eta) \rho_{0} \mu_{0}^{-1}$, the last integral in (4.3.6) is indeed finite. This completes the proof.

Now we have a fundamental estimate for our counting function $N_{\mu}(r: \dot{g})$, that is

Proposition 4.3.2. Let $\tau=\tau_{\mathbf{Q}}(G, \sigma)$ be as before and $s$ a real number with $s>(2+\tau) \rho_{0} \mu_{0}^{-1}$. Let $l$ denote the semisimple $\mathbf{Q}$-rank of $H$. Then given a Siegel domain $\mathcal{S}$ for $\Gamma \backslash G_{\mathbf{R}}$, we have

(1)

$$
N_{\mu}(r: \dot{g}) \prec r^{s} a_{G}(g)^{(2-\tau) \rho_{G}}\left(1+\left|\log a_{G}(g)^{\rho_{G}}\right|^{l}\right), \quad g \in \mathcal{S}, r>0,
$$


(2)

$$
\sum_{\gamma \in \Gamma_{H} \backslash \Gamma} \varphi_{\mu}(\gamma g)^{-s} \prec a_{G}(g)^{(2-\tau) \rho_{G}}\left(1+\left|\log a_{G}(g)^{\rho_{G}}\right|^{l}\right), \quad g \in \mathcal{S} .
$$

Proof. This is a direct consequence of Corollary 4.3.1, Proposition 4.3.1 and Lemma 4.3.3.

Theorem 4.3.1. $\quad$ Put $\tau=\tau_{\mathbf{Q}}(G, \sigma)$. Let $r$ be a positive real number. Then $\dot{g} \mapsto N_{\mu}(r: \dot{g})$ is a measurable function on $\Gamma \backslash G_{\mathbf{R}}$ with values in $\mathbf{N}$. For any $u>1$ with $(2-\tau) u<2$, we have

$$
\int_{\Gamma \backslash G_{\mathbf{R}}}\left(N_{\mu}(r: \dot{g})\right)^{u} d \dot{g}<+\infty .
$$

Proof. Let $\chi_{r}$ be the characteristic function of the set $\left\{x \in G_{\mathbf{R}} \mid \varphi_{\mu}(x) \leqslant\right.$ $r\}$ in $G_{\mathbf{R}}$. Then we easily see that

$$
N_{\mu}(r: \dot{g})=\sum_{\gamma \in \Gamma_{H} \backslash \Gamma} \chi_{r}(\gamma g), \quad r>0, \dot{g} \in \Gamma \backslash G_{\mathbf{R}} .
$$

For each $\gamma \in \Gamma$, the function $g \mapsto \chi_{r}(\gamma g)$ is a measurable function on $G_{\mathbf{R}}$; hence the function $g \mapsto N_{\mu}(r: \dot{g})$ is measurable also. From Corollary 4.3.2 the value $N_{\mu}(r: \dot{g})$ is a natural number. Let $\mathcal{S}=\omega A_{G, \mathbf{R}}^{+}(t) K$ be a Siegel domain for $\Gamma \backslash G_{\mathbf{R}}$. From the previous proposition, we have

$$
\int_{\mathcal{S}}\left(N_{\mu}(r: \dot{g})\right)^{u} d g \prec \int_{A_{G, \mathbf{R}}^{+}(t)} a^{(2-\tau) u \rho_{G}}\left(1+\left|\log \left(a^{\rho_{G}}\right)\right|^{l}\right)^{u} a^{-2 \rho_{G}} d a .
$$

The finiteness of the last integral follows from Lemma 1.4.1 because $(2-\tau) u-$ $2<0$ by assumption. By Lemma 1.4.2, we have the conclusion.

Remark 4.3.1. In the arguments of this section we do not use the condition in Proposition 2.4.2 (2) on $\left(G_{\mathbf{R}}, H_{\mathbf{R}}\right)$.

\section{$\S 5 . \quad L^{p}$-Estimate of Poincaré Series}

Given an arithmetic subgroup $\Gamma$ of $G_{\mathbf{Q}}$, using the results obtained in the previous section, we establish an $L^{p}$-estimate of the function $G_{s}$. 


\section{$\S 5.1$. Formulation of theorem}

Theorem 5.1.1. Let $\tau=\tau_{\mathbf{Q}}(G, \sigma) \in[0,2]$ be as in 1.5. Let $s \in \mathbf{C}$ with $\operatorname{Re}(s)>\rho_{0}(\tau+1)$. For any real number $p$ with $p \geqslant 1, p(2-\tau)<2$, we have

$$
\int_{\Gamma \backslash G_{\mathbf{R}}}\left|G_{s}(x)\right|^{p} d \dot{x}<+\infty .
$$

Corollary 5.1.1. Assume $\tau=\tau_{\mathbf{Q}}(G, \sigma)>1$. Then there exists $\delta \in$ $(0,+\infty]$ such that for any $s \in \mathbf{C}$ with $\operatorname{Re}(s)>\rho_{0}(\tau+1)$

$$
G_{s} \in \bigcap_{0 \leqslant \epsilon<\delta} L^{2+\epsilon}\left(\Gamma \backslash G_{\mathbf{R}}\right) .
$$

In particular we have $G_{s} \in L^{2}\left(\Gamma \backslash G_{\mathbf{R}}\right)$.

Proof. This is a direct consequence of Theorem 5.1.1. Indeed, when $\tau=2$, the second condition $(2-\tau) p<2$ is empty; hence we may take $\delta=+\infty$ in this case. When $1<\tau<2$, put $\delta=2(2-\tau)^{-1}-2$; then we have $\delta>0$. For any $0 \leqslant \epsilon<\delta$, we see that the number $p=2+\epsilon$ satisfies the condition $p>1$, $p(2-\tau)<2$.

The proof of the theorem is given in Subsection 5.3. Before that we need some preparation.

\section{$\S 5.2$. The estimate of truncated part}

As before, let $\varphi_{\mu}$ be any one of gauge functions on $G_{\mathbf{R}}$.

Proposition 5.2.1. Let $f$ be a measurable function on $H_{\mathbf{R}} \backslash G_{\mathbf{R}}$ such that

$$
\int_{H_{\mathbf{R}} \backslash G_{\mathbf{R}}}|f(x)|^{p} d \dot{x}<+\infty
$$

for all $p \geqslant 1$. Furthermore we assume that there exists a positive $r>0$ such that the support of $f$ is contained in the set $D(r)=\left\{\dot{x} \in H_{\mathbf{R}} \backslash G_{\mathbf{R}} \mid \varphi_{\mu}(x) \leqslant r\right\}$.

Put

$$
p_{f}(x)=\sum_{\gamma \in \Gamma_{H} \backslash \Gamma} f(\gamma x), \quad x \in G_{\mathbf{R}} .
$$

Then $p_{f}(x)$ converges absolutely for almost all $x \in G_{\mathbf{R}}$ and $p_{f} \in L^{p}\left(\Gamma \backslash G_{\mathbf{R}}\right)$ for any $p$ satisfying $p \geqslant 1,(2-\tau) p<2$ with $\tau=\tau_{\mathbf{Q}}(G, \sigma)$. 
Proof. Our proof is similar to [20, Theorem 3.4]. By analogous computation as that in the proof of Proposition 3.1.1, we have

$$
\int_{\Gamma \backslash G_{\mathbf{R}}}\left(\sum_{\gamma \in \Gamma_{H} \backslash \Gamma}|f(\gamma x)|\right) d \dot{x}=\operatorname{vol}\left(\Gamma_{H} \backslash H_{\mathbf{R}}\right) \int_{H_{\mathbf{R}} \backslash G_{\mathbf{R}}}|f(x)| d \dot{x} .
$$

Since this is finite from the assumption, we have

$$
\sum_{\gamma \in \Gamma_{H} \backslash \Gamma}|f(\gamma x)|<+\infty
$$

almost everywhere in $x$; we also have $p_{f} \in L^{1}\left(H_{\mathbf{R}} \backslash G_{\mathbf{R}}\right)$. Thus we may consider $p_{f}(x)$ as an almost everywhere defined measurable function on $\Gamma \backslash G_{\mathbf{R}}$.

To show that $p_{f} \in L^{p}\left(\Gamma \backslash G_{\mathbf{R}}\right)$ for any $p$ such that $p>1, p(2-\tau)<2$, it suffices to prove the following.

Claim 5.2.1. $\quad$ There exists a constant $C_{f}$ such that

$$
\left|\int_{\Gamma \backslash G_{\mathbf{R}}} p_{f}(x) \varphi(x) d \dot{x}\right| \leqslant C_{f}\|\varphi\|_{L^{q}\left(\Gamma \backslash G_{\mathbf{R}}\right)}, \quad \varphi \in C_{\mathrm{c}}\left(\Gamma \backslash G_{\mathbf{R}}\right)
$$

with $q>1$ determined from $p$ by $p^{-1}+q^{-1}=1$.

Take a positive number $u$ such that $u>p$ and $u(2-\tau)<2$. We can see that such a $u$ exists: if $\tau=2$, then any $u$ with $u>p$ is sufficient; if $\tau<2$, then from assumption we have $1<p<2(2-\tau)^{-1}$. Hence we can take a $u$ with $p<u<2(2-\tau)^{-1}$.

For $u$ fixed above, we define real numbers $v, q^{\prime}$ and $p^{\prime}$ by the relations

$$
\frac{1}{u}+\frac{1}{v}=1, \quad q^{\prime}=\frac{q}{v}, \quad \frac{1}{p^{\prime}}+\frac{1}{q^{\prime}}=1 .
$$

Then $v>1, q^{\prime}>1$ and $p^{\prime}>1$. Indeed, from $u>p$, we have $u^{-1}<p^{-1}$; hence $v^{-1}=1-u^{-1}>1-p^{-1}=q^{-1}$, which in turn gives $q^{\prime}=q v^{-1}>1$. Since $u>p>1$, we have $v>1$. From $p^{\prime-1}+q^{\prime-1}=1$ and $q^{\prime}>1$ we have $p^{\prime}>1$.

Now we start the evaluation

$$
\begin{aligned}
\left|\int_{\Gamma \backslash G_{\mathbf{R}}} p_{f}(x) \varphi(x) d \dot{x}\right| & =\left|\int_{\Gamma \backslash G_{\mathbf{R}}} \sum_{\gamma \in \Gamma_{H} \backslash \Gamma} f(\gamma x) \varphi(\gamma x) d \dot{x}\right| \\
& =\left|\int_{\Gamma_{H} \backslash G_{\mathbf{R}}} f(x) \varphi(x) d \dot{x}\right| \\
& =\left|\int_{H_{\mathbf{R}} \backslash G_{\mathbf{R}}}\left(\int_{\Gamma_{H} \backslash H_{\mathbf{R}}} f(h x) \varphi(h x) d \dot{h}\right) d \dot{x}\right|
\end{aligned}
$$




$$
\begin{aligned}
& =\left|\int_{H_{\mathbf{R}} \backslash G_{\mathbf{R}}} f(x)\left(\int_{\Gamma_{H} \backslash H_{\mathbf{R}}} \varphi(h x) d \dot{h}\right) d \dot{x}\right| \\
& \leqslant \int_{H_{\mathbf{R}} \backslash G_{\mathbf{R}}}|f(x)| \int_{\Gamma_{H} \backslash H_{\mathbf{R}}}|\varphi(h x)| d \dot{h} d \dot{x} \\
& =\int_{H_{\mathbf{R}} \backslash G_{\mathbf{R}}}|f(x)| \int_{\Gamma_{H} \backslash H_{\mathbf{R}}} \chi_{r}(x)|\varphi(h x)| d \dot{h} d \dot{x},
\end{aligned}
$$

where $\chi_{r}$ is the characteristic function of the set $D(r)$. We apply the Hölder inequality to the last formula of (5.2.2) to have

$$
\begin{aligned}
\int_{H_{\mathbf{R}} \backslash G_{\mathbf{R}}}|f(x)| & \int_{\Gamma_{H} \backslash H_{\mathbf{R}}} \chi_{r}(x)|\varphi(h x)| d \dot{h} d \dot{x} \\
\leqslant & \left\{\int_{H_{\mathbf{R}} \backslash G_{\mathbf{R}}}|f(x)|^{p^{\prime}} d \dot{x}\right\}^{1 / p^{\prime}}\left\{\int_{H_{\mathbf{R}} \backslash G_{\mathbf{R}}} \chi_{r}(x)\right. \\
& \left.\times\left(\int_{\Gamma_{H} \backslash H_{\mathbf{R}}}|\varphi(h x)| d \dot{h}\right)^{q^{\prime}} d \dot{x}\right\}^{1 / q^{\prime}} .
\end{aligned}
$$

We use the Hölder inequality again to get

$$
\begin{aligned}
\int_{\Gamma_{H} \backslash H_{\mathbf{R}}}|\varphi(h x)| d \dot{h} & \leqslant\left(\int_{\Gamma_{H} \backslash H_{\mathbf{R}}}|\varphi(h x)|^{q^{\prime}} d \dot{h}\right)^{1 / q^{\prime}}\left(\int_{\Gamma_{H} \backslash H_{\mathbf{R}}} d \dot{h}\right)^{1 / p^{\prime}} \\
& =\operatorname{vol}\left(\Gamma_{H} \backslash H_{\mathbf{R}}\right)^{1 / p^{\prime}}\left(\int_{\Gamma_{H} \backslash H_{\mathbf{R}}}|\varphi(h x)|^{q^{\prime}} d \dot{h}\right)^{1 / q^{\prime}}
\end{aligned}
$$

From (5.2.2), (5.2.3) and (5.2.4), we have

$$
\begin{aligned}
& \left|\int_{\Gamma \backslash G_{\mathbf{R}}} p_{f}(x) \varphi(x) d \dot{x}\right| \\
& \leqslant\|f\|_{p^{\prime}} \operatorname{vol}\left(\Gamma_{H} \backslash H_{\mathbf{R}}\right)^{1 / p^{\prime}}\left(\int_{\Gamma_{H} \backslash G_{\mathbf{R}}} \chi_{r}(x)|\varphi(x)|^{q^{\prime}} d \dot{x}\right)^{1 / q^{\prime}} \\
& =\|f\|_{p^{\prime}} \operatorname{vol}\left(\Gamma_{H} \backslash H_{\mathbf{R}}\right)^{1 / p^{\prime}}\left(\int_{\Gamma \backslash G_{\mathbf{R}}} \sum_{\gamma \in \Gamma_{H} \backslash \Gamma} \chi_{r}(\gamma x)|\varphi(x)|^{q^{\prime}} d \dot{x}\right)^{1 / q^{\prime}} \\
& =\|f\|_{p^{\prime}} \operatorname{vol}\left(\Gamma_{H} \backslash H_{\mathbf{R}}\right)^{1 / p^{\prime}}\left(\int_{\Gamma \backslash G_{\mathbf{R}}} N_{\mu}(r: \dot{x})|\varphi(x)|^{q^{\prime}} d \dot{x}\right)^{1 / q^{\prime}} .
\end{aligned}
$$


Here \|\|$_{p}$ denotes the $p$-norm on the space $L^{p}\left(H_{\mathbf{R}} \backslash G_{\mathbf{R}}\right)$. By Hölder's inequality, we have

$$
\begin{aligned}
& \int_{\Gamma \backslash G_{\mathbf{R}}} N_{\mu}(r: \dot{x})|\varphi(x)|^{q^{\prime}} d \dot{x} \\
& \quad \leqslant\left(\int_{\Gamma \backslash G_{\mathbf{R}}}\left(N_{\mu}(r: \dot{x})\right)^{u} d \dot{x}\right)^{1 / u}\left(\int_{\Gamma \backslash G_{\mathbf{R}}}|\varphi(x)|^{q^{\prime} v} d \dot{x}\right)^{1 / v} .
\end{aligned}
$$

Noting $q^{\prime} v=q$, we have the desired inequality (5.2.1) combining (5.2.5) and (5.2.6); the constant $C_{f}$ in (5.2.1) is defined by

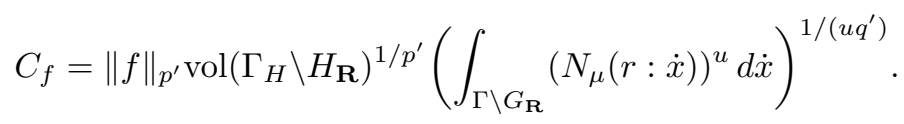

By assumption $\|f\|_{p^{\prime}}$ is finite. Since $u$ is so chosen that $u>p>1$ and $u(2-\tau)<2$, Theorem 4.3.1 tells the last factor in $C_{f}$ is finite. Hence $C_{f}$ is finite. This completes the proof of Claim 5.2.1.

\section{§5.3. The proof of Theorem 5.1.1}

Now we begin the proof of Theorem 5.1.1. Take a function $\psi \in$ $C^{\infty}\left(H_{\mathbf{R}} \backslash G_{\mathbf{R}} / K\right)$ such that

(a) $0 \leqslant \psi(x) \leqslant 1$ for $x \in G_{\mathbf{R}}$,

(b) $\psi\left(\exp \left(t Y_{0}\right)\right)=1$ for $t>2$

(c) and $\psi\left(\exp \left(t Y_{0}\right)\right)=0$ for $0 \leqslant t<1$.

Set

$$
\begin{aligned}
& { }^{\prime} \phi_{s}^{(2)}(g)=\phi_{s}^{(2)}(g) \psi(g), \quad g \in G_{\mathbf{R}}, \\
& { }^{\prime \prime} \phi_{s}^{(2)}(g)=\phi_{s}^{(2)}(g)(1-\psi(g)), \quad g \in G_{\mathbf{R}}-S .
\end{aligned}
$$

Then we have that the functions ' $\phi_{s}^{(2)}$ and ${ }^{\prime \prime} \phi_{s}^{(2)}$ are both left $H_{\mathbf{R}}$-invariant and right $K$-invariant. Moreover the function ' $\phi_{s}^{(2)}$ is everywhere smooth on $G_{\mathbf{R}}$. Consider the two infinite series:

$$
\begin{aligned}
{ }^{\prime} G_{s}(x) & =\sum_{\gamma \in \Gamma_{H} \backslash \Gamma}{ }^{\prime} \phi_{s}^{(2)}(\gamma x), \\
{ }^{\prime} G_{s}(x) & =\sum_{\gamma \in \Gamma_{H} \backslash \Gamma}{ }^{\prime \prime} \phi_{s}^{(2)}(\gamma x), \quad x \in G_{\mathbf{R}} .
\end{aligned}
$$

To have an $L^{p}$-estimate of the second series, we need 
Claim 5.3.1. The function $f=" \phi_{s}^{(2)}$ satisfies the assumption in Proposition 5.2.1. Namely " $\phi_{s}^{(2)}$ belongs to $L^{p}\left(H_{\mathbf{R}} \backslash G_{\mathbf{R}}\right)$ for any $p \geqslant 1$, and there exists a constant $r>0$ such that $\varphi_{\mu}(x)>r$ implies $" \phi_{s}^{(2)}(x)=0$.

Proof. Let $x \in G_{\mathbf{R}}$ and decompose it as $x=h \exp \left(t Y_{0}\right) k$ with $h \in H_{\mathbf{R}}$, $t \geqslant 0$ and $k \in K$. By the definition of " $\phi_{s}^{(2)}$, we have " $\phi_{s}^{(2)}(x)=0$ if $t>2$. Hence if we put $r=\sup \left\{\varphi_{\mu}\left(\exp \left(t Y_{0}\right)\right) \mid 0 \leqslant t \leqslant 2\right\}$, then $\varphi_{\mu}(x)>r$ implies ${ }^{\prime \prime} \phi_{s}^{(2)}(x)=0$. For any $p \geqslant 1$, we have

$$
\begin{aligned}
\left.\left.\int_{H_{\mathbf{R}} \backslash G_{\mathbf{R}}}\right|^{\prime \prime} \phi_{s}^{(2)}(x)\right|^{p} d \dot{x} & =\left.\int_{0}^{\infty}\right|^{\prime \prime} \phi_{s}^{(2)}\left(\left.\exp \left(t Y_{0}\right)\right|^{p} \gamma_{\mathfrak{h} \backslash \mathfrak{g}}(t) d t\right. \\
& \leqslant \int_{0}^{2}\left|\phi_{s}^{(2)}\left(\exp \left(t Y_{0}\right)\right)\right|^{p} \gamma_{\mathfrak{h} \backslash \mathfrak{g}}(t) d t .
\end{aligned}
$$

In view of Corollary 2.4.1 and the explicit form of $\gamma_{\mathfrak{h} \backslash \mathfrak{g}}(t)$, we have the estimates $\left|\phi_{s}^{(2)}\left(\exp \left(t Y_{0}\right)\right)\right| \prec|\log (t)|$ and $\gamma_{\mathfrak{h}} \backslash \mathfrak{g}(t) \prec t^{m_{\lambda}^{+}+m_{2 \lambda}^{+}}=t$ on the interval $0<t<2$. Hence the last integral in (5.2.7) is dominated by a positive constant multiple of the integral of $|\log t|^{p} t$ on the interval $0<t<2$, that is easily seen to be finite.

By Claim 5.3.1, we can apply Proposition 5.2.1 to have that the infinite series " $G_{s}(x)$ converges absolutely for almost everywhere in $x$ and, as a function of $\dot{x} \in \Gamma \backslash G_{\mathbf{R}}$, it belongs to the space $L^{p}\left(\Gamma \backslash G_{\mathbf{R}}\right)$ for any $p$ with $p>1, p(2-\tau)<$ 2. Since $G_{s}(x)={ }^{\prime} G_{s}(x)+{ }^{\prime \prime} G_{s}(x)$, the proof of our theorem is reduced to the following claim.

Claim 5.3.2. The infinite series ' ${ }^{\prime} G_{s}(x)$ converges for all $x$ and as a function of $\dot{x} \in \Gamma \backslash G_{\mathbf{R}}$, it belongs to $L^{p}\left(\Gamma \backslash G_{\mathbf{R}}\right)$ for $p$ satisfying $p>1, p(2-\tau)<$ 2 .

Proof. By using Corollary 2.4.1 and (4.2.1), we can easily verify that

$$
\left.\right|^{\prime} \phi_{s}^{(2)}(x) \mid \prec \varphi_{\mu}(x)^{-\mu_{0}^{-1}\left(\operatorname{Re}(s)+\rho_{0}\right)}, \quad x \in G_{\mathbf{R}} .
$$

By using Proposition 4.3.2 (2), this in turn implies that

$$
\begin{aligned}
\left.\sum_{\gamma \in \Gamma_{H} \backslash \Gamma}\right|^{\prime} \phi_{s}^{(2)}(\gamma x) \mid & \prec \sum_{\gamma \in \Gamma_{H} \backslash \Gamma} \varphi_{\mu}(\gamma x)^{-\mu_{0}^{-1}\left(\operatorname{Re}(s)+\rho_{0}\right)} \\
& \prec a_{G}(x)^{(2-\tau) \rho_{G}}\left(1+\left|\log a_{G}(x)^{\rho_{G}}\right|^{l}\right), \quad x \in \mathcal{S}
\end{aligned}
$$

for $\mathcal{S}$ a Siegel domain for $\Gamma \backslash G_{\mathbf{R}}$. By this estimate, combined with the integration formula (1.4.1) and Lemma 1.4.2, the problem is reduced to the estimation 
of the integral

$$
\int_{A_{G, \mathbf{R}}^{+}(t)} a^{(p(2-\tau)-2) \rho_{G}}\left(1+\left|\log a^{\rho_{G}}\right|^{l}\right)^{p} d a .
$$

It is finite if $p(2-\tau)<2$ from Lemma 1.4.1.

\section{§6. Meromorphic Continuation and Functional Equation of $\boldsymbol{G}_{s}$}

We investigate the analytic property of $G_{s}$ using the spectral decomposition of $L^{2}\left(\Gamma \backslash G_{\mathbf{R}} / K\right)$. The apparatus of Eisenstein series for general $G_{\mathbf{R}}$ satisfying the assumption in the preceeding section is a bit too heavy compared with the expected gain. So we consider only the case when $G_{\mathbf{R}} \cong S U(n, 1)$ and $H_{\mathbf{R}} \cong U(n-1,1)$. As we explained briefly in the introduction, we put the $\mathbf{Q}$ structures on $G_{\mathbf{R}}$ and on $H_{\mathbf{R}}$ as follows. Let $E$ be an imaginary quadratic field in C. Let $G=S U(\Phi)$, where $\Phi: V \times V \rightarrow E$ is a non-degenerate Hermitian form on an $(n+1)$-dimensional $E$-vector space of Witt index 1 with signature $(n+, 1-)$. Let $v_{o}^{+}$be a vector in $V$ such that $\Phi\left(v_{o}^{+}, v_{o}^{+}\right)>0$ and $H$ the stabilizer in $G$ of the one dimensional subspace $E v_{o}^{+}$. Then we have $G_{\mathbf{R}} \cong S U(n, 1)$ and $H_{\mathbf{R}} \cong U(n-1,1)$. A maximal compact subgroup $K$, that is admissible with respect to $H$, is obtained as the stabilizer in $G_{\mathbf{R}}$ of a vector $v^{-} \in V_{\mathbf{R}}:=V \otimes_{\mathbf{Q}} \mathbf{R}$ with $\Phi\left(v^{-}, v^{-}\right)<0, \Phi\left(v^{-}, v_{o}^{+}\right)=0$.

The two vectors $v_{o}^{+}$and $v^{-}$span a hyperbolic plane in $V_{\mathbf{R}} \cong \mathbf{C}^{n+1}$. Take isotropic vectors $f^{\prime}$ and $f^{\prime \prime}$ in that hyperbolic plane such that $\Phi\left(f^{\prime}, f^{\prime \prime}\right)=1$. Let $Q_{\mathfrak{p}, \mathfrak{q}}$ be the stabilizer of the line $\mathbf{C} f^{\prime}$ and $A_{\mathfrak{p}, \mathfrak{q}}$ the $\mathbf{R}$-split torus corresponding to $\left\{f^{\prime}, f^{\prime \prime}\right\}$. Then we easily see that the number $\rho_{0}$ equals $n$ in the present situation.

Let $e^{\prime}$ and $e^{\prime \prime}$ be vectors in $V$ such that $\Phi\left(e^{\prime}, e^{\prime}\right)=\Phi\left(e^{\prime \prime}, e^{\prime \prime}\right)=0, \Phi\left(e^{\prime}, e^{\prime \prime}\right)$ $=1$; hence $E e^{\prime}+E e^{\prime \prime}$ is a hyperbolic plane. We further assume that $v_{o}^{+}$is orthogonal to $E e^{\prime}+E e^{\prime \prime}$. Let $P_{G}$ be the stabilizer in $G$ of the one dimensional isotropic subspace $E e^{\prime}$ and $A_{G}$ the subgroup of $P_{G}$ consisting of those elements $a$ such that

$$
a\left(e^{\prime}\right)=t e^{\prime}, \quad a\left(e^{\prime \prime}\right)=t^{-1} e^{\prime \prime}, \quad a \mid\left(E e^{\prime}+E e^{\prime \prime}\right)^{\perp}=\mathrm{id}
$$

for some scalar $t$. Then $P_{G}$ is a minimal parabolic $\mathbf{Q}$-subgroup of $G$ and $A_{G}$ a split component of $P_{G}$. Put $P_{H}=P_{G} \cap H$ and $A_{H}=A_{G}$. Thus we obtain a minimal parabolic Q-subgroup $P_{H}$ of $H$ and its split component $A_{H}$.

Lemma 6.0.1. Let $\alpha$ be the character of $A_{G}$, that assingns the scalar $t$ in (6.0) to $a \in A_{G}$. 
(i) We have

$$
\rho_{G}=n \alpha, \quad \rho_{H}=(n-1) \alpha .
$$

(ii) The number $\tau=\tau_{\mathbf{Q}}(G, \sigma)$ is given by

$$
\tau=2\left(1-\frac{1}{n}\right) .
$$

Proof. The restricted root system for $\left(A_{G}, G\right)$ is of non-reduced $B C_{1}$. The multiplicity of the short root $\alpha$ is $2 n-2$ and that of the long one $2 \alpha$ is 1 . Hence $2 \rho_{G}=(2 n-2) \alpha+2 \alpha=2 n \alpha$. Similarly we have $2 \rho_{H}=2(n-1) \alpha$. By definition $\tau$ is the supremum of those $\eta \in[0,2]$ such that $2(n-1)-\eta n \geqslant 0$; hence $\tau=2\left(1-n^{-1}\right)$ as desired.

Hence $\tau>1$ if and only if $n \geqslant 3$. Therefore when $n \geqslant 3$ we can apply Theorem 5.1.1 and Corolary 3.2.1 to have theorems (i) and (ii) in the introduction. The aim of this section is to prove theorem (iii) in the introduction. From now on we assume $\tau>1$, or equivalently $n \geqslant 3$.

\section{§6.1. Eisenstein series}

Let $C=\left\{\kappa_{1}, \ldots, \kappa_{r}\right\}$ be a complete set of representatives of the double coset space $\Gamma \backslash G_{\mathbf{Q}} / P_{G, \mathbf{Q}}$; we assume $\kappa_{1}=e$. Then we can take a Siegel domain $\mathcal{S}=\mathcal{S}_{\omega, t_{0}}$ for $\Gamma \backslash G_{\mathbf{R}}$ so that

$$
G_{\mathbf{R}}=\bigcup_{i=1}^{r} \Gamma \kappa_{i} \mathcal{S}
$$

and $\omega$ is a fundamental domain for $\Gamma \cap{ }^{\circ} P_{G, \mathbf{R}} \backslash^{\circ} P_{G, \mathbf{R}}$.

For each $i$ with $1 \leqslant i \leqslant r$, put $P^{i}=\kappa_{i} P_{G} \kappa_{i}^{-1}$ and $A^{i}=\kappa_{i} A_{G} \kappa_{i}^{-1} ; P^{i}$ is a minimal parabolic Q-subgroup of $G$ and $A^{i}$ a maximal Q-split torus in the radical of $P^{i}$. Let $N_{G}$ and $N^{i}$ be the unipotent radicals of $P_{G}$ and $P^{i}$ respectively. Replacing $\left(P_{G}, A_{G}\right)$ by $\left(P^{i}, A^{i}\right)$, we get the counterpart of the function $a_{G}$ and that of the character $2 \rho_{G}$, which we denote by $a^{i}$ and $2 \rho^{i}$ respectively. Then we have

$$
a_{G}\left(\kappa_{i}^{-1} g\right) a_{G}\left(\kappa_{i}^{-1}\right)^{-1}=\kappa_{i}^{-1} a^{i}(g) \kappa_{i}, \quad g \in G_{\mathbf{R}} .
$$

Let $\alpha$ and $\alpha^{i}$ be simple roots of $A_{G}$ and $A^{i}$ corresponding to $P_{G}=P^{1}$ and $P^{i}$ respectively. Then we have

$$
a_{G}\left(\kappa_{i}^{-1} g\right)^{\alpha} a_{G}\left(\kappa_{i}^{-1}\right)^{-\alpha}=a^{i}(g)^{\alpha^{i}}, \quad g \in G_{\mathbf{R}} .
$$


Let $Y \in \operatorname{Lie}\left(A_{G, \mathbf{R}}\right)$ and $Y^{i} \in \operatorname{Lie}\left(A_{\mathbf{R}}^{i}\right)$ be the vectors defined by $\alpha(Y)=1$ and $\alpha^{i}\left(Y^{i}\right)=1$.

In the subsequent paragraphs, we need an integration formula associated with the Iwasawa decomposition. Let $d n$ be a Haar measure of $N_{G, \mathbf{R}}$. We can take $d n$ in such a way that

$$
\int_{G_{\mathbf{R}}} \varphi(g) d g=\int_{N_{G}, \mathbf{R}} d n \int_{K} d k \int_{-\infty}^{+\infty} e^{-2 \rho_{G}(Y) t} \varphi(n \exp (t Y) k) d t
$$

holds for $\varphi \in C_{\mathrm{c}}\left(G_{\mathbf{R}}\right)$. Here $d g, d t$ and $d k$ are as in 1.3. Let $d n^{i}$ be the Haar measure of $N_{\mathbf{R}}^{i}$ that corresponds to $d n$ by the isomorphism $N_{G, \mathbf{R}} \cong N_{\mathbf{R}}^{i}$ defined by $n \mapsto k_{i} n k_{i}^{-1}$, where $k_{i} \in K$ is the element of $K$ such that $\kappa_{i} k_{i}^{-1} \in$ $A_{G, \mathbf{R}} N_{G, \mathbf{R}}$; let $\operatorname{vol}\left(\Gamma \cap N_{\mathbf{R}}^{i} \backslash N_{\mathbf{R}}^{i}\right)$ be the volume of $\Gamma \cap N_{\mathbf{R}}^{i} \backslash N_{\mathbf{R}}^{i}$ with respect to the measure $d n^{i}$.

The $K$-spherical Eisenstein series is defined by

$$
E^{i}(\nu: g)=\sum_{\gamma \in \Gamma \cap P_{\mathbf{R}}^{i} \backslash \Gamma} a^{i}(\gamma g)^{\nu \alpha^{i}+\rho^{i}}, \quad g \in G_{\mathbf{R}}, \nu \in \mathbf{C} .
$$

Below, we list up some properties of Eisenstein series that is necessary later:

(i) The series $E^{i}(\nu: g)$ converges absolutely and locally uniformly with respect to $g$ if $\operatorname{Re}(\nu)>\rho_{0}$, to give rise to an automorphic form on $\Gamma \backslash G_{\mathbf{R}}$. The function $\nu \mapsto E^{i}(\nu)$ is meromorphically continued to all of $\mathbf{C}$ holomorphic on $\operatorname{Re}(\nu)=0$.

(ii) There exists a positive integer $N$ and a positive-valued locally bounded function $c(\nu)$ defined for $\nu$ at which $E^{i}(\nu)$ is regular such that

$$
\left|E^{i}(\nu: g) * D_{g}\right| \leqslant c(\nu) a^{j}(g)^{N \alpha^{j}}, \quad g \in \kappa_{j} \mathcal{S}
$$

for any $D \in U\left(\mathfrak{g}_{\mathbf{C}}\right)$ and $\nu \in \mathbf{C}$ away from the poles.

(iii) For $j$ with $1 \leqslant j \leqslant r$, let $E_{j}^{i}(\nu: g)$ be the constant term of $E^{i}(\nu: g)$ along $P^{j}$, that is

$$
E_{j}^{i}(\nu: g)=\frac{1}{\operatorname{vol}\left(\Gamma \cap N_{\mathbf{R}}^{i} \backslash N_{\mathbf{R}}^{i}\right)} \int_{\Gamma \cap N_{\mathbf{R}}^{j} \backslash N_{\mathbf{R}}^{j}} E^{i}\left(\nu: n^{j} g\right) d \dot{n}^{j} .
$$

Then there exists a meromorphic function $c_{i j}(\nu)$ such that

$$
E_{j}^{i}(\nu: g)=\delta_{i j} a^{j}(g)^{\nu \alpha^{j}+\rho^{j}}+c_{i j}(\nu) a^{j}(g)^{-\nu \alpha^{j}+\rho^{j}} .
$$


(iv) We have

$$
E^{i}(\nu) * \Omega=\left(\nu^{2}-\rho_{0}^{2}\right) E^{i}(\nu), \quad \nu \in \mathbf{C} .
$$

(v) Let $B$ be a compact subset of $\mathbf{C}$ such that $E^{i}(\nu)$ is holomorphic in a neighborhood of $B$. Let $\epsilon$ be a positive number such that $\sup _{\nu \in B}|\operatorname{Re}(\nu)| \leqslant$ $1-\epsilon$. Then

$$
\left|E^{i}(\nu: g) * X_{g}\right| \prec a^{j}(g)^{(2-\epsilon) \rho^{j}}, \quad \nu \in B, g \in \kappa_{j} \mathcal{S}
$$

for $X \in U\left(\mathfrak{g}_{\mathbf{C}}\right)$.

The properties (i), (ii) and (iii) are well known and are found in [11]. The presence of the number $\rho_{0}$ in (iv) is explained by the equality $\rho_{0}^{2}=\rho_{G}(Y)^{2}=$ $\rho^{i}\left(Y^{i}\right)^{2}=n^{2}$. (v) is proved in [19, Lemma A.2.2].

Proposition 6.1.1. Let $W$ be the set of $\nu \in \mathbf{C}$ at which $E^{i}(\nu)$ 's are regular. There exists a positive constant $\epsilon$ such that the integral

$$
\left\langle\delta_{D_{o}}, E^{i}(\nu)\right\rangle=\int_{\Gamma_{H} \backslash H_{\mathbf{R}}} E^{i}(\nu: h) d \dot{h}
$$

converges absolutely and locally uniformly for $\nu \in W$ with $|\operatorname{Re}(\nu)|<\epsilon$. The function $\nu \mapsto\left\langle\delta_{D_{o}}, E^{i}(\nu)\right\rangle$, that is given by the integral above for $\nu \in W$ in a neighborhood of the imaginary axis, is continued to a meromorphic function on C holomorphic on $\operatorname{Re}(\nu)=0$.

Proof. If $H$ is $\mathbf{Q}$-anisotropic then the manifold $\Gamma_{H} \backslash H_{\mathbf{R}}$ is compact; hence all assertions in the proposition follow from the properties of Eisenstein series listed above. Now we consider the case when $H$ contains a one dimensional Q-split torus.

Since we use the truncation operator in the following discussion, we recall its definition here; for details see [26, Section 3]: It is given, for a positive real number $t$ and a continuous function $\varphi$ on $\Gamma \backslash G_{\mathbf{R}}$, by the formula

$$
\begin{aligned}
\left(\Lambda^{t} \varphi\right)(g)= & \varphi(g)-\sum_{i=1}^{r} \sum_{\xi \in \Gamma \cap P_{\mathbf{R}}^{i} \backslash \Gamma}\left(\int_{\Gamma \cap N_{\mathbf{R}}^{i} \backslash N_{\mathbf{R}}^{i}} \varphi(n \xi g) d \dot{n}\right) \\
& \times \chi^{i}\left(\log a^{i}(\xi g)-t^{i} Y^{i}\right), \quad g \in G_{\mathbf{R}},
\end{aligned}
$$

where $\chi^{i}$ is the characteristic function on $\operatorname{Lie}\left(A_{\mathbf{R}}^{i}\right)$ of the subset $\left\{Y \in \operatorname{Lie}\left(A_{\mathbf{R}}^{i}\right) \mid \alpha^{i}(Y)>0\right\}$ and

$$
t^{i}=\log (t)-\log a_{G}\left(\kappa_{i}^{-1}\right)^{\alpha}, \quad 1 \leqslant i \leqslant r .
$$


Let $N$ be a positive real number. Using [26, Proposition 3.8], combined with the estimate (6.1.2), we have

$$
\left|\Lambda^{t} E^{i}(\nu: g)\right| \leqslant C \cdot c(\nu) a^{j}(g)^{-N \alpha^{j}}, \quad g \in \kappa_{j} \mathcal{S}, \nu \in W
$$

for a constant $C>0$. From this we conclude that the function $\nu \mapsto \sup _{g \in G_{\mathbf{R}}} \wedge^{t} E^{i}(\nu)$ is locally bounded for $\nu$ away from the poles of $E^{i}(\nu)$. Hence the integral

$$
\int_{\Gamma_{H} \backslash H_{\mathbf{R}}} \Lambda^{t} E^{i}(\nu: h) d \dot{h}
$$

converges absolutely and locally uniformly for $\nu \in W$ to define a meromorphic function of $\nu$.

Let $C_{H}=\left\{\kappa_{H}^{q} \mid 1 \leqslant q \leqslant r_{H}\right\}$ be a complete set of representatives for $\Gamma_{H} \backslash H_{\mathbf{Q}} / P_{H, \mathbf{Q}}$ and $\omega_{H}$ a fundamental domain for $\Gamma \cap^{\circ} P_{H, \mathbf{R}} \backslash{ }^{\circ} P_{H, \mathbf{R}}$ and $\mathcal{S}_{\omega_{H}, t_{H}}^{H}$ a Siegel domain for $\Gamma_{H} \backslash H_{\mathbf{R}}$. Now we take the truncation parameter $t>0$ large enough so that

(a) $t>t_{0}$ and $t>t_{H}$,

(b) $\kappa_{H}^{q} \mathcal{S}_{\omega_{H}, t}^{H} \cap \kappa_{H}^{q^{\prime}} \mathcal{S}_{\omega_{H}, t}^{H}=\emptyset$ if $q \neq q^{\prime}$,

(c) the quotient map $\pi_{H}: H_{\mathbf{R}} \rightarrow \Gamma_{H} \backslash H_{\mathbf{R}}$ is injective on each $\kappa_{H}^{q} \mathcal{S}_{\omega_{H}, t}^{H}$

(d) if $\gamma \in \Gamma, \delta, \quad \delta^{\prime} \in P_{G, \mathbf{Q}}$ and $\gamma \kappa_{i} \delta \mathcal{S}_{\omega, t} \cap \kappa_{j} \delta^{\prime} \mathcal{S}_{\omega, t_{0}} \neq \emptyset$, then $i=j$ and $\gamma \in \Gamma \cap P_{\mathbf{Q}}^{i}$.

Let $\mathcal{D}$ be the complement of the union of $\pi_{H}\left(\kappa_{H}^{q} \mathcal{S}_{\omega_{H}, t}^{H}\right)$ 's in $\Gamma_{H} \backslash H_{\mathbf{R}}$; it is a relatively compact subset. Then, from (b) and (c), we have

$$
\begin{aligned}
\int_{\Gamma_{H} \backslash H_{\mathbf{R}}} & \left(E^{i}(\nu: h)-\Lambda^{t} E^{i}(\nu: h)\right) d \dot{h} \\
= & \int_{\mathcal{D}}\left(E^{i}(\nu: h)-\Lambda^{t} E^{i}(\nu: h)\right) d \dot{h} \\
& +\sum_{q=1}^{r_{H}} \int_{\kappa_{H}^{q} \mathcal{S}_{\omega_{H}^{H}, t}^{H}}\left(E^{i}(\nu: h)-\Lambda^{t} E^{i}(\nu: h)\right) d h
\end{aligned}
$$

Since $\mathcal{D}$ is relatively compact, the first term in the right hand side of (6.1.8) converges absolutely and locally uniformly for $\nu \in W$ to define a meromorphic function of $\nu$. Now we examine the remaining terms.

If we choose the $\omega$ that enters in the definition of $\mathcal{S}$ so that $\omega_{H} \subset \omega$, then we have $\mathcal{S}_{\omega_{H}, t}^{H} \subset \mathcal{S}_{\omega, t}$. For a given index $1 \leqslant q \leqslant r_{H}$, we can find $\kappa_{j} \in C, \gamma \in \Gamma$ 
and $\delta \in P_{G, \mathbf{Q}}$ such that $\kappa_{H}^{q}=\gamma^{-1} \kappa_{j} \delta$. Then we obviously have

$$
\kappa_{H}^{q} \mathcal{S}_{\omega_{H}, t}^{H} \subset \gamma^{-1} \kappa_{j} \delta \mathcal{S}_{\omega, t} .
$$

Now we claim

$$
E^{i}(\nu: g)-\Lambda^{t} E^{i}(\nu: g)=E_{j}^{i}(\nu: \gamma g), \quad g \in \gamma^{-1} \kappa_{j} \delta \mathcal{S}_{\omega, t} .
$$

If $\chi^{i}\left(\log a^{i}(\xi g)-t^{i} Y^{i}\right) \neq 0$ with $g \in \gamma^{-1} \kappa_{j} \delta \mathcal{S}_{\omega, t}, 1 \leqslant i \leqslant r$ and $\xi \in \Gamma$, then using (5.1.1), we have

$$
a_{G}\left(\kappa_{i}^{-1} \xi g\right)^{\alpha}=a^{i}(\xi g)^{\alpha^{i}} a_{G}\left(\kappa_{i}^{-1}\right)^{\alpha}>e^{t^{i}} a_{G}\left(\kappa_{i}^{-1}\right)^{\alpha}=t .
$$

This is equivalent to saying that $\kappa_{i}^{-1} \xi g \in{ }^{\circ} P_{G, \mathbf{R}} A_{G, \mathbf{R}}^{+}(t) K$. Since $\omega$ is a fundamental domain for $\Gamma \cap P_{G, \mathbf{Q}} \backslash^{\circ} P_{G, \mathbf{R}}$, we can find a $\delta^{\prime} \in \Gamma \cap P_{G, \mathbf{Q}}$ such that $\delta^{\prime-1} \kappa_{i}^{-1} \xi g \in \mathcal{S}_{\omega, t}$. Thus we have $g \in \xi^{-1} \kappa_{i} \delta^{\prime} \mathcal{S}_{\omega, t}$. Hence we have

$$
g \in \gamma^{-1} \kappa_{j} \delta \mathcal{S}_{\omega, t} \cap \xi^{-1} \kappa_{i} \delta^{\prime} \mathcal{S}_{\omega, t} .
$$

By (d) above, we then have $i=j$ and $\gamma\left(\Gamma \cap P_{\mathbf{Q}}^{j}\right)=\xi\left(\Gamma \cap P_{\mathbf{Q}}^{j}\right)$. This means that only those terms for $i=j$ and $\xi=\gamma$ are non zero in the summation of (6.1.6) if $g \in \gamma^{-1} \kappa_{j} \delta \mathcal{S}_{\omega, t}$. Hence we have (6.1.10).

Noting (6.1.9) and (6.1.10) and then using the formula (6.1.3), we have

$$
\begin{aligned}
& \int_{\kappa_{H}^{q} \mathcal{S}_{\omega_{H}, t}^{H}}\left(E^{i}(\nu: h)-\Lambda^{t} E^{i}(\nu: h)\right) d h \\
& =\int_{\mathcal{S}_{\omega_{H}, t}^{H}} E_{j}^{i}\left(\nu: \gamma \kappa_{H}^{q} h\right) d h \\
& =\int_{\mathcal{S}_{\omega_{H}, t}^{H}}\left(\delta_{i j} a^{j}\left(\gamma \kappa_{H}^{q} h\right)^{\nu \alpha^{j}+\rho^{j}}+c_{i j}(\nu) a^{j}\left(\gamma \kappa_{H}^{q} h\right)^{-\nu \alpha^{j}+\rho^{j}}\right) d h .
\end{aligned}
$$

From (6.1.1), noting $a_{H}(h)=a_{G}(h), h \in H_{\mathbf{R}}$ and $\kappa_{j}^{-1} \gamma \kappa_{H}^{q}=\delta \in P_{G, \mathbf{Q}}$, we have

$$
\begin{aligned}
a^{j}\left(\gamma \kappa_{H}^{q} h\right)^{ \pm \nu \alpha^{j}+\rho^{j}} & =a_{G}\left(\kappa_{j}^{-1} \gamma \kappa_{H}^{q} h\right)^{ \pm \nu \alpha+\rho_{G}} a_{G}\left(\kappa_{j}^{-1}\right)^{\mp \nu \alpha-\rho_{G}} \\
& =a_{G}(\delta h)^{ \pm \nu \alpha+\rho_{G}} a_{G}\left(\kappa_{j}^{-1}\right)^{\mp \nu \alpha-\rho_{G}} \\
& =a_{H}(h)^{ \pm \nu \alpha+\rho_{G}} a_{G}\left(\kappa_{j}^{-1}\right)^{\mp \nu \alpha-\rho_{G}} .
\end{aligned}
$$

Substituting the final formula in (6.1.12) for $a^{j}\left(\gamma \kappa_{H}^{q} h\right)^{ \pm \nu \alpha^{j}+\rho^{j}}$ in the righthand side of (6.1.11), and after that using the integration formula (1.4.1), we 
have

$$
\begin{aligned}
& \int_{\kappa_{H}^{q} \mathcal{S}_{\omega_{H}^{H}, t}^{H}}\left(E^{i}(\nu: h)-\Lambda^{t} E^{i}(\nu: h)\right) d h \\
& =\left(\int_{\omega_{H}} d h\right) a_{G}\left(\kappa_{j}^{-1}\right)^{-\rho_{G}} \\
& \quad \times\left(\delta_{i j} a_{G}\left(\kappa_{j}^{-1}\right)^{-\nu \alpha} \int_{A_{H, \mathbf{R}}^{+}(t)} a_{H}^{\nu \alpha+\rho_{G}-2 \rho_{H}} d a_{H}\right. \\
& \left.\quad+c_{i j}(\nu) a_{G}\left(\kappa_{j}^{-1}\right)^{\nu \alpha} \int_{A_{H, \mathbf{R}}^{+}(t)} a_{H}^{-\nu \alpha+\rho_{G}-2 \rho_{H}} d a_{H}\right) .
\end{aligned}
$$

Here since $\tau>1$, we have $\rho_{G}(Y)<2 \rho_{H}(Y)$; hence for $\nu \in \mathbf{C}$ with $|\operatorname{Re}(\nu)|<$ $\left(2 \rho_{H}-\rho_{G}\right)(Y)$, the integrals in the right-hand side of (6.1.13) are finite and are evaluated as

$$
\begin{aligned}
\int_{A_{H, \mathbf{R}}^{+}(t)} a_{H}^{ \pm \nu \alpha+\rho_{G}-2 \rho_{H}} d a_{H} & =\int_{\log t}^{\infty} e^{t\left( \pm \nu+\left(\rho_{G}-2 \rho_{H}\right)(Y)\right)} d t \\
& =-\frac{t^{ \pm \nu+\left(\rho_{G}-2 \rho_{H}\right)(Y)}}{ \pm \nu+\left(\rho_{G}-2 \rho_{H}\right)(Y)}
\end{aligned}
$$

The last expression is holomorphic on $\mathbf{C}$ away from $\pm\left(\rho_{G}-2 \rho_{H}\right)(Y) \in \mathbf{R}-\{0\}$. This establishes the convergence of the integral in the left-hand side of (6.1.13) and its meromorphy with respect to $\nu \in W$ to complete the proof.

\section{§6.2. Spectral decomposition of $G_{s}$}

Since we assume $\tau>1$, i.e., $n \geqslant 3$, we have $G_{s} \in L^{2}\left(\Gamma \backslash G_{\mathbf{R}}\right)$ for $s \in \mathbf{C}$ with $\operatorname{Re}(s)>(\tau+1) \rho_{0}$. Hence, following [20], we can use the spectral decomposition of $L^{2}\left(\Gamma \backslash G_{\mathbf{R}}\right)$ to analyze the function $G_{s}(x)$.

We recall the spectral decomposition of $L^{2}\left(\Gamma \backslash G_{\mathbf{R}}\right)^{K}=L^{2}\left(\Gamma \backslash G_{\mathbf{R}} / K\right)$, the $K$-invariant part of $L^{2}\left(\Gamma \backslash G_{\mathbf{R}}\right)$. The negative of the Casimir element $-\Omega$ acts on $C^{\infty}\left(\Gamma \backslash G_{\mathbf{R}}\right)$ from the right to give rise to the densely defined selfadjoint operator $\triangle$ on $L^{2}\left(\Gamma \backslash G_{\mathbf{R}} / K\right)$ that is positive in the sense that $\langle\triangle \varphi, \varphi\rangle \geqslant 0$ for $\varphi \in C_{\mathrm{c}}^{\infty}\left(\Gamma \backslash G_{\mathbf{R}} / K\right)$. Let $\Lambda_{\Gamma}$ be the set of eigenvalues of $\triangle$ on $L^{2}\left(\Gamma \backslash G_{\mathbf{R}} / K\right)$. Then it is known that $\Lambda_{\Gamma}$ is a countable subset of nonnegative real numbers without accumulation points and that for each $\lambda \in \Lambda_{\Gamma}$ the $\lambda$-eigenvectors are $C^{\infty}$-functions and make up a finite dimensional space. So we can enumerate the elements of $\Lambda_{\Gamma}$ as

$$
0=\lambda_{0}<\lambda_{1} \leqslant \lambda_{2} \leqslant \cdots \leqslant \lambda_{n} \leqslant \cdots
$$


in such a way that each $\lambda \in \Lambda_{\Gamma}$ occurs in the sequence above exactly $m(\lambda)$ times, where $m(\lambda)$ denotes the dimension of the $\lambda$-eigenspace. Note that the eigenspace for $\lambda_{0}=0$ is the space of constant functions, that is one dimensional. Let $\mu_{n}$ be a complex number such that $\lambda_{n}=\rho_{0}^{2}-\mu_{n}^{2}$; since $\lambda_{n} \geqslant 0$, we have $\mu_{n} \in \sqrt{-1} \mathbf{R} \cup\left[-\rho_{0}, \rho_{0}\right]$. We can take an orthonormal family of eigenvectors $\left\{\varphi_{n}\right\}$ consisting of automorphic forms on $\Gamma \backslash G_{\mathbf{R}}$ such that

$$
\triangle \varphi_{n}=\lambda_{n} \varphi_{n}, \quad n \in \mathbf{N} .
$$

Especially we have

$$
\varphi_{0}(\dot{x})=\frac{1}{\operatorname{vol}\left(\Gamma \backslash G_{\mathbf{R}}\right)^{1 / 2}}, \quad \dot{x} \in \Gamma \backslash G_{\mathbf{R}} .
$$

Let $L_{\mathrm{d}}^{2}\left(\Gamma \backslash G_{\mathbf{R}} / K\right)$ be the closed span of $\left\{\varphi_{n}\right\}$ and $L_{\mathrm{c}}^{2}\left(\Gamma \backslash G_{\mathbf{R}} / K\right)$ the orthogonal complement of $L_{\mathrm{d}}^{2}\left(\Gamma \backslash G_{\mathbf{R}} / K\right)$ in $L^{2}\left(\Gamma \backslash G_{\mathbf{R}} / K\right)$.

Let us recall the decomposition of the continuous part $L_{\mathrm{c}}^{2}\left(\Gamma \backslash G_{\mathbf{R}} / K\right)$. Let $\mathcal{L}^{0}$ be the space of $r$-tuples of compactly supported $C^{\infty}$-functions $\mathbf{f}=\left(f^{i}\right)_{1 \leqslant i \leqslant r}$,

$$
f^{i}: \sqrt{-1} \mathbf{R} \rightarrow \mathbf{C},
$$

such that

(a) $f^{j}(-\nu)=\sum_{i=1}^{r} c_{i j}(\nu) f^{i}(\nu), \quad \nu \in \sqrt{-1} \mathbf{R}, 1 \leqslant i \leqslant r$,

(b) $\int_{\sqrt{-1} \mathbf{R}}\left|f^{i}(\nu)\right|^{2} d|\nu|<+\infty, \quad 1 \leqslant i \leqslant r$.

We define a Hermitian inner product on $\mathcal{L}^{0}$ by putting the associated norm

$$
\|\mathbf{f}\|_{\mathcal{L}}^{2}=\frac{1}{4 \pi} \sum_{i=1}^{r} \int_{\sqrt{-1} \mathbf{R}}\left|f^{i}(\nu)\right|^{2} d|\nu|
$$

for $\mathbf{f}=\left(f^{i}\right)$. For any $\mathbf{f}=\left(f^{i}\right) \in \mathcal{L}^{0}$, set

$$
\operatorname{Eis}(\mathbf{f}: \dot{x})=\frac{1}{4 \pi} \sum_{i=1}^{r} \int_{\sqrt{-1} \mathbf{R}} E^{i}(\nu: \dot{x}) f^{i}(\nu) d|\nu|, \quad \dot{x} \in \Gamma \backslash G_{\mathbf{R}} .
$$

Then a basic fact is that the function $\dot{x} \mapsto \operatorname{Eis}(\mathbf{f}: \dot{x})$ belongs to $L_{\mathrm{c}}^{2}\left(\Gamma \backslash G_{\mathbf{R}} / K\right)$ and its $L^{2}$-norm is given by

$$
\|\operatorname{Eis}(\mathbf{f})\|_{L^{2}}=\|\mathbf{f}\|_{\mathcal{L}}
$$

Now let $\mathcal{L}$ be the Hilbert space completion of $\mathcal{L}^{0}$. Then the map $\mathbf{f} \mapsto \operatorname{Eis}(\mathbf{f})$, that is at first defined on $\mathcal{L}^{0}$, can be extended to all of $\mathcal{L}$ to give rise to an isometry from $\mathcal{L}$ onto $L_{\mathrm{c}}^{2}\left(\Gamma \backslash G_{\mathbf{R}} / K\right)$. 
From the facts recalled above, we have the spectral decomposition of a given $\varphi \in L^{2}\left(\Gamma \backslash G_{\mathbf{R}} / K\right)$

$$
\varphi=\sum_{n=0}^{\infty}\left(\varphi \mid \varphi_{n}\right)_{L^{2}} \cdot \varphi_{n}+\operatorname{Eis}(\mathbf{f})
$$

with a unique $\mathbf{f} \in \mathcal{L}$, which is called the continuous part of $\varphi$. To have an expression of $\mathbf{f}$ for $\varphi$, we can use the following.

Proposition 6.2.1. $\quad$ Let $\varphi$ be a measurable function on $\Gamma \backslash G_{\mathbf{R}}$ such that

$$
\varphi \in \bigcap_{0 \leqslant \epsilon<\delta} L^{2+\epsilon}\left(\Gamma \backslash G_{\mathbf{R}}\right)
$$

with some $\delta>0$. Then for each $i$ with $1 \leqslant i \leqslant r$, the integral

$$
f_{\varphi}^{i}(\nu)=\int_{\Gamma \backslash G_{\mathbf{R}}} \varphi(x) \overline{E^{i}(\nu: x)} d \dot{x}, \quad \nu \in \sqrt{-1} \mathbf{R}
$$

converges absolutely. The $r$-tuple $\mathbf{f}_{\varphi}=\left(f_{\varphi}^{i}\right)_{1 \leqslant i \leqslant r}$ belongs to the space $\mathcal{L}$ and $\operatorname{Eis}\left(\mathbf{f}_{\varphi}\right)$ gives the continuous part of $\varphi$.

Proof. [20, Proposition A.2.3].

Now we apply the theory recalled above to our $G_{s}$. We first have

Proposition 6.2.2. Let $s \in \mathbf{C}$ be such that $\operatorname{Re}(s)>(\tau+1) \rho_{0}$.

(1) For $n \in \mathbf{N}$, we have

$$
\left(G_{s} \mid \varphi_{n}\right)_{L^{2}}=\frac{\left\langle\delta_{D_{o}}, \overline{\varphi_{n}}\right\rangle}{\mu_{n}^{2}-s^{2}}
$$

(2) For each $i$ with $1 \leqslant i \leqslant r$ and $\nu \in \sqrt{-1} \mathbf{R}$, the integral

$$
f^{i}(s: \nu)=\int_{\Gamma \backslash G_{\mathbf{R}}} G_{s}(x) \overline{E^{i}(\nu: x)} d \dot{x}
$$

converges absolutely and equals

$$
\frac{\left\langle\delta_{D_{o}}, E^{i}(-\nu)\right\rangle}{\nu^{2}-s^{2}} .
$$

The cotinuous part of $G_{s}$ is given by $\mathbf{f}(s)=\left(f^{i}(s:-)\right)_{1 \leqslant i \leqslant r} \in \mathcal{L}$. 
(3) The spectral decomposition of $G_{s}$ is

$$
G_{s}=\sum_{n=0}^{\infty} \frac{\left\langle\delta_{D_{o}}, \overline{\varphi_{n}}\right\rangle}{\mu_{n}^{2}-s^{2}} \varphi_{n}-\frac{1}{4 \pi} \sum_{i=1}^{r} \int_{\mathbf{R}} \frac{\left\langle\delta_{D_{o}}, E^{i}(-\sqrt{-1} \nu)\right\rangle}{\nu^{2}+s^{2}} E^{i}(\sqrt{-1} \nu) d \nu .
$$

Proof. (1) This follows from Proposition 3.3.1 since $G_{s}$ is in $L^{2}\left(\Gamma \backslash G_{\mathbf{R}}\right)$. (2) Since we have Corollary 5.1.1, we can use Proposition 6.2.1 to have that the integral $f^{i}(s: \nu)$ converges absolutely and that the continuous part of $G_{s}$ is given by $\operatorname{Eis}(\mathbf{f}(s))$ with $\mathbf{f}(s)=\left(f^{i}(s:-)\right) \in \mathcal{L}$. To compute $f^{i}(s: \nu)$ we appeal to Theorem 3.2.1. The property (v) of the Eisenstein series implies that $E^{i}(\nu)$ with $\nu \in \sqrt{-1} \mathbf{R}$ satisfies the condition in Theorem 3.2.1. We have

$$
\int_{\Gamma \backslash G_{\mathbf{R}}} G_{s}(x)\left(\triangle+s^{2}-\rho_{0}^{2}\right) \overline{E^{i}(\nu: x)} d \dot{x}=-\int_{\Gamma_{H} \backslash H_{\mathbf{R}}} \overline{E^{i}(\nu: h)} d \dot{h} .
$$

Since $\nu$ is purely imaginary we have $\overline{E^{i}(\nu: x)}=E^{i}(-\nu: x)$. By using the differential equation (vi) of $E^{i}(\nu)$, we have $\triangle E^{i}(-\nu: x)=\left(\rho_{0}^{2}-\nu^{2}\right) E^{i}(-\nu: x)$. Combining these remarks together, we get the desired formula of $f^{i}(s: \nu)$.

Lemma 6.2.1. For any $z, w \in \mathbf{C}$, we have

$$
\frac{\left|z^{2}-w^{2}\right|}{|z|^{2}+1} \leqslant 1+|w|^{2}
$$

Proof. Obvious.

\section{Theorem 6.2.1.}

(1) Let $B$ be a compact subset of $\mathbf{C}$ that does not contain any of points $\pm \mu_{n}$, $n \in \mathbf{N}$. Then we have

$$
\sup _{s \in B}\left(\sum_{n=0}^{\infty} \frac{\left|\left\langle\delta_{D_{o}}, \overline{\varphi_{n}}\right\rangle\right|^{2}}{\left|\mu_{n}^{2}-s^{2}\right|^{2}}\right)<+\infty
$$

(2) For $s \in \mathbf{C}$ and $1 \leqslant i \leqslant r$, we define the function $f^{i}(s): \sqrt{-1} \mathbf{R} \rightarrow \mathbf{C}$ by putting

$$
f^{i}(s: \nu)=\frac{\left\langle\delta_{D_{o}}, E^{i}(-\nu)\right\rangle}{\nu^{2}-s^{2}}, \quad \nu \in \sqrt{-1} \mathbf{R} .
$$

Then the r-tuple $\mathbf{f}(s)=\left(f^{i}(s)\right)_{1 \leqslant i \leqslant r}$ belongs to the space $\mathcal{L}$ if $\operatorname{Re}(s)>0$. Let $B^{\prime}$ be a compact subset of $\operatorname{Re}(s)>0$. Then we have

$$
\sup _{s \in B^{\prime}}\|\mathbf{f}(s)\|_{\mathcal{L}}<+\infty
$$


Proof. Let $s_{0}$ be a point on the domain $\operatorname{Re}(s)>(\tau+1) \rho_{0}$.

(1) Since $B$ is a compact subset disjoint from $\left\{ \pm \mu_{n} \mid n \in \mathbf{N}\right\}$, there exists a constant $c_{0}$ such that

$$
\frac{\left|\mu_{n}\right|^{2}+1}{\left|\mu_{n}^{2}-s^{2}\right|} \leqslant c_{0}, \quad s \in B, n \in \mathbf{N} .
$$

Hence, in view of Lemma 6.2.1, we have

$$
\frac{\left|\mu_{n}^{2}-s_{0}^{2}\right|}{\left|\mu_{n}^{2}-s^{2}\right|} \leqslant c_{0} \frac{\left|\mu_{n}^{2}-s_{0}^{2}\right|}{\left|\mu_{n}\right|^{2}+1} \leqslant c_{0}\left(1+\left|s_{0}\right|^{2}\right), \quad s \in B .
$$

Using this, we have

$$
\begin{aligned}
\sup _{s \in B}\left(\sum_{n=0}^{\infty} \frac{\left|\left\langle\delta_{D_{o}}, \overline{\varphi_{n}}\right\rangle\right|^{2}}{\left|\mu_{n}^{2}-s^{2}\right|^{2}}\right) & \leqslant c_{0}^{2}\left(1+\left|s_{0}\right|^{2}\right)^{2} \sum_{n=0}^{\infty} \frac{\left|\left\langle\delta_{D_{o}}, \overline{\varphi_{n}}\right\rangle\right|^{2}}{\left|\mu_{n}^{2}-s_{0}^{2}\right|^{2}} \\
& \leqslant c_{0}^{2}\left(1+\left|s_{0}\right|^{2}\right)^{2}\left\|G_{s_{0}}\right\|_{L^{2}}^{2}<+\infty
\end{aligned}
$$

by the Bessel inequality and Proposition 6.2.2.

(2) Since $B^{\prime}$ is a compact subset disjoint from the imaginary axis, there exists a constant $c_{1}$ such that

$$
\frac{|\nu|^{2}+1}{\left|\nu^{2}-s^{2}\right|} \leqslant c_{1}, \quad s \in B^{\prime}, \nu \in \sqrt{-1} \mathbf{R} .
$$

Using this and the inequality in Lemma 6.2.1, we have

$$
\begin{aligned}
& \sup _{s \in B^{\prime}} \int_{\nu \in \sqrt{-1} \mathbf{R}} \frac{\left|\left\langle\delta_{D_{o}}, E^{i}(-\nu)\right\rangle\right|^{2}}{\left|\nu^{2}-s^{2}\right|^{2}} d|\nu| \\
& \quad \leqslant c_{1}^{2}\left(1+\left|s_{0}\right|^{2}\right)^{2} \int_{\sqrt{-1} \mathbf{R}} \frac{\left|\left\langle\delta_{D_{o}}, E^{i}(-\nu)\right\rangle\right|^{2}}{\left|\nu^{2}-s_{0}^{2}\right|^{2}} d|\nu| \\
& \quad \leqslant c_{1}^{2}\left(1+\left|s_{0}\right|^{2}\right)^{2} 4 \pi\left\|\mathbf{f}\left(s_{0}\right)\right\|_{\mathcal{L}}^{2} .
\end{aligned}
$$

This last expression is finite because of Proposition 6.2.2. Thus the condition (b) for $\mathbf{f}(s)$ to belong to $\mathcal{L}$ is satisfied. Obviously $f^{i}(s: \nu)$ depends on $s$ analytically; hence the condition (a) is valid for any $s$ with $\operatorname{Re}(s)>0$ because Proposition 6.2.2 implies that it is true for $s$ in the half plane $\operatorname{Re}(s)>(\tau+1) \rho_{0}$. Thus we have $\mathbf{f}(s) \in \mathcal{L}$ for $\operatorname{Re}(s)>0$.

\section{§6.3. Meromorphic continuation and functional equation of $\boldsymbol{G}_{s}$}

Now we have the main theorem of this section: 


\section{Theorem 6.3.1.}

(1) For any $\varphi \in C_{\mathrm{c}}^{\infty}\left(\Gamma \backslash G_{\mathbf{R}}\right)$, the function $s \mapsto\left\langle G_{s}, \varphi\right\rangle$, that is at first defined on the half plane $\operatorname{Re}(s)>\rho_{0}$, is meromorphically continued to the whole $\mathbf{C}$ with the functional equation in the sense of distributions:

$$
-G_{s}+G_{-s}=\frac{1}{4 s} \sum_{i=1}^{r}\left(\left\langle\delta_{D_{o}}, E^{i}(-s)\right\rangle E^{i}(s)+\left\langle\delta_{D_{o}}, E^{i}(s)\right\rangle E^{i}(-s)\right) .
$$

(2) If $\operatorname{Re}(s)>0$ and $s$ outside the set of poles, then $G_{s} \in L^{2}\left(\Gamma \backslash G_{\mathbf{R}}\right)$.

(3) The poles of $G_{s}$ for $\operatorname{Re}(s) \geqslant 0, s \neq 0$ are simple and are contained in the set of $s$ such that there is a $K$-invariant automorphic form $\varphi \in L^{2}\left(\Gamma \backslash G_{\mathbf{R}} / K\right)$ satisfying $\triangle \varphi=\left(\rho_{0}^{2}-s^{2}\right) \varphi$ and $\left\langle\delta_{D_{o}}, \varphi\right\rangle \neq 0$. We have

$$
\operatorname{Res}_{s=\rho_{0}} G_{s}=\frac{\operatorname{vol}\left(\Gamma_{H} \backslash H_{\mathbf{R}}\right)}{\operatorname{vol}\left(\Gamma \backslash G_{\mathbf{R}}\right)} \frac{1}{2 \rho_{0}},
$$

a constant function.

Proof. We can prove this in a similar way as [20, Proposition 4.3] using Proposition 6.1.1 to overcome a difficulity caused by the noncompactness of $\Gamma_{H} \backslash H_{\mathbf{R}}$.

For $s \in \mathbf{C}$ with $\operatorname{Re}(s)>0$, put

$$
\begin{aligned}
G_{s}^{\mathrm{d}} & =\sum_{n=0}^{\infty} \frac{\left\langle\delta_{D_{o}}, \overline{\varphi_{n}}\right\rangle}{\mu_{n}^{2}-s^{2}} \varphi_{n}, \\
G_{s}^{\mathrm{c}} & =\operatorname{Eis}(\mathbf{f}(s)),
\end{aligned}
$$

where $\mathbf{f}(s)$ is as in Theorem 6.2.1. From Theorem 6.2.1, the expression of $G_{s}^{\text {d }}$ converges in $L^{2}\left(\Gamma \backslash G_{\mathbf{R}} / K\right)$ for any $s \in \mathbf{C}$ with $s \neq \pm \mu_{n}, n \in \mathbf{N}$ locally uniformly to give rise to an $L^{2}\left(\Gamma \backslash G_{\mathbf{R}} / K\right)$-valued meromorphic function on all of $s \in \mathbf{C}$, that is holomorphic away from the set of $\pm \mu_{n}$ 's. Theorem 6.2.1 also implies that the expression of $G_{s}^{\mathrm{c}}$ defines an element of $L^{2}\left(\Gamma \backslash G_{\mathbf{R}} / K\right)$ and the resulting function $s \mapsto G_{s}^{\mathrm{c}}$ gives an $L^{2}\left(\Gamma \backslash G_{\mathbf{R}} / K\right)$-valued holomorphic function on $\operatorname{Re}(s)>0$. Since $G_{s}=G_{s}^{\mathrm{d}}+G_{s}^{\mathrm{c}}$ for $\operatorname{Re}(s)>(\tau+1) \rho_{0}$ by Proposition 6.2 .2 , we thus obtained an analytic continuation of $G_{s}$ to the half plane $\operatorname{Re}(s)>0$.

Let $\varphi \in C_{\mathrm{c}}^{\infty}\left(\Gamma \backslash G_{\mathbf{R}} / K\right)$. Our next task is to obtain an expression of $\left\langle G_{s}^{\mathrm{c}}, \varphi\right\rangle$ that is valid on a neighborhood of the imaginary axis as well as on $\operatorname{Re}(s)>0$. Now put

$$
\hat{\varphi}^{i}(\nu)=\int_{\Gamma \backslash G_{\mathbf{R}}} E^{i}(\nu: x) \varphi(x) d \dot{x}, \quad \nu \in \mathbf{C} .
$$


Then we have

$$
\left\langle G_{s}^{\mathrm{c}}, \varphi\right\rangle=\frac{-1}{4 \pi \sqrt{-1}} \sum_{i=1}^{r} \int_{\mathbf{R}} \frac{\left\langle\delta_{D_{o}}, E^{i}(-\sqrt{-1} \nu)\right\rangle}{\nu^{2}+s^{2}} \hat{\varphi}^{i}(\sqrt{-1} \nu) d \nu
$$

for $\operatorname{Re}(s)>0$. Let us take an $\epsilon$ and an $R$ such that $E^{i}(\nu)$ is holomorphic on the domain $|\operatorname{Re}(\nu)|<\epsilon,|\operatorname{Im}(\nu)|<R$ and the integrals defining $\left\langle\delta_{D_{o}}, E^{i}(-\nu)\right\rangle$ are absolutely convergent on that domain (see Proposition 6.1.1); then for any $i$ the function $\nu \mapsto\left\langle\delta_{D_{o}}, E^{i}(-\nu)\right\rangle E^{i}(\nu)$ is holomorphic on the domain $0<\operatorname{Re}(\nu)<\epsilon,|\operatorname{Im}(\nu)|<R$. Let $\mathcal{C}_{R, \epsilon}$ be the path that comes along the imaginary axis from $-\sqrt{-1} \infty$ to $-\sqrt{-1} R$, rounds around the origin along the boundary of the rectangle having vertices $-\sqrt{-1} R,-\sqrt{-1} R+\epsilon,+\sqrt{-1} R+\epsilon$ and $+\sqrt{-1} R$ through these points in this order and then goes away to $+\sqrt{-1} \infty$ along the imaginary axis again. Let $D_{R, \epsilon}$ be the domain defined by $|\operatorname{Re}(s)|<\epsilon / 2,|\operatorname{Im}(s)|<R / 2$. Let $s \in D_{R, \epsilon}$ with $\operatorname{Re}(s)>0$. Then applying the residue theorem, we have

$$
\begin{aligned}
\left\langle G_{s}^{\mathrm{c}}, \varphi\right\rangle= & \frac{-1}{4 \pi \sqrt{-1}} \sum_{i=1}^{r} \int_{\mathbf{R}} \frac{\left\langle\delta_{D_{o}}, E^{i}(-\sqrt{-1} \nu)\right\rangle}{\nu^{2}+s^{2}} \hat{\varphi}^{i}(\sqrt{-1} \nu) d \nu \\
= & \frac{-1}{4 \pi \sqrt{-1}} \sum_{i=1}^{r}\left\{\int_{\mathcal{C}_{R, \epsilon}} \frac{\left\langle\delta_{D_{o}}, E^{i}(-\nu)\right\rangle}{-\nu^{2}+s^{2}} \hat{\varphi}^{i}(\nu) d \nu-2 \pi \sqrt{-1} \operatorname{Res}_{\nu=s}\right. \\
& \left.\times\left(\frac{\left\langle\delta_{D_{o}}, E^{i}(-\nu)\right\rangle}{-\nu^{2}+s^{2}} \hat{\varphi}^{i}(\nu)\right)\right\} \\
= & \frac{-1}{4 \pi \sqrt{-1}} \int_{\mathcal{C}_{R, \epsilon}} \frac{\left\langle\delta_{D_{o}}, E^{i}(-\nu)\right\rangle}{-\nu^{2}+s^{2}} \hat{\varphi}^{i}(\nu) d \nu \\
& -\frac{1}{4 s} \sum_{i=1}^{r}\left\langle\delta_{D_{o}}, E^{i}(-s)\right\rangle \hat{\varphi}^{i}(s) .
\end{aligned}
$$

The first term in the last line of (6.3.3) is holomorphic on all of $D_{R, \epsilon}$, so is the second one except the origin. By letting $\epsilon$ and $R$ vary, we have an analytic continuation of $\left\langle G_{s}^{\mathrm{c}}, \varphi\right\rangle$ on a neighborhood of the imaginary axis. From the last form of (6.3.3), we have the formula

$$
\left\langle G_{s}^{\mathrm{c}}, \varphi\right\rangle-\left\langle G_{-s}^{\mathrm{c}}, \varphi\right\rangle=\frac{-1}{4 s} \sum_{i=1}^{r}\left(\left\langle\delta_{D_{o}}, E^{i}(-s)\right\rangle \hat{\varphi}^{i}(s)+\left\langle\delta_{D_{o}}, E^{i}(s)\right\rangle \hat{\varphi}^{i}(-s)\right)
$$

that is valid for $s$ in a neighborhood of the imaginary axis. With this formula, we can extend $\left\langle G_{s}^{\mathrm{c}}, \varphi\right\rangle$ meromorphically to all of $\mathbf{C}$ noting Proposition 6.1.1. 
At this point we have finished the proof of (1) and (2). As for (3), it suffices to note the following point: From the expression (6.3.1) and (6.3.3) combined with Proposition 6.1.1, the poles of $\left\langle G_{s}, \varphi\right\rangle$ lying on $\operatorname{Re}(s)=0, s \neq 0$ come from $G_{s}^{\mathrm{d}}$; hence they are at $s= \pm \mu_{n}$ and are all simple.

Remark 6.3.1. When $G_{\mathbf{R}} \cong \operatorname{Spin}(n, 2)$, the analysis in this section should work with slight modification if the Q-rank of $G$ is one. Here the Eisenstein series should be replaced by the Eisenstein series obtained by 'lifting' of cusp forms on the Levi conponent of the Siegel parabolic subgroups. If the Q-rank of $G$ is two, there appears various terms corresponding to the other types of parabolic subgroups. We also have to analyse these new terms.

\section{$\S 7 . \quad$ Behavior of the Current $\partial \bar{\partial} \boldsymbol{G}_{s}$}

The aim of this section is to give systems of differential equations for the current $G_{s}$ and another related current $\Psi_{s}$. (see Theorem 7.6.1). The first 4 subsections are preliminaries. In Subsection 7.5, we introduce the Poincaré series $\Psi_{s}$, which is constructed from a vector-valued function $\psi_{s}$ on $G_{\mathbf{R}}-H_{\mathbf{R}} K$ obtained by applying a Schmid operator to $\phi_{s}^{(2)}$.

Retaining the assumptions (i), (ii) and (iii) on $\left(G_{\mathbf{R}}, H_{\mathbf{R}}\right)$ in Section 3 , we consider the two cases:

(O) $(\mathfrak{g}, \mathfrak{h})$ is orthogonal type, i.e., it is isomorphic to $(\mathfrak{s o}(n, 2), \mathfrak{s o}(n-1,2))$ with $n \geqslant 2$;

(U) $(\mathfrak{g}, \mathfrak{h})$ is unitary type, i.e., it is isomorphic to $(\mathfrak{s u}(n, 1), \mathfrak{s}(\mathfrak{u}(n-1,1) \times \mathfrak{u}(1)))$ with $n \geqslant 2$.

\section{§7.1. Radial part of some differential operators}

Our goal of this subsection is Lemma 7.1.2, which is indispensable in the computation of the radial part of various differential operators. Before proving Lemma 7.1.2 we first give an auxiliary lemma to fix a system of vectors in $\mathfrak{g}_{\mathbf{C}}$ with some bracket-relations.

Let $\lambda_{0}$ be the unique root in $\Psi^{+}$such that $m_{\lambda_{0}}^{+}=1 ; \lambda_{0}=2 \lambda$ if $(\mathfrak{g}, \mathfrak{h})$ is of type $(\mathrm{U})$ and $\lambda_{0}=\lambda$ if $(\mathfrak{g}, \mathfrak{h})$ is of type $(\mathrm{O})$. Let $\tilde{Y}_{0}$ be the vector in $\mathfrak{a}_{\mathfrak{p}, \mathfrak{q}}$ determined by $\lambda_{0}\left(\tilde{Y}_{0}\right)=2$; it equals $Y_{0}$ or $2 Y_{0}$ according as $(\mathfrak{g}, \mathfrak{h})$ is of type (U) or $(\mathrm{O})$. The number $\lambda\left(\tilde{Y}_{0}\right)$ appears in many formulas in this section; so we put $c_{\mathfrak{g}}=\lambda\left(\tilde{Y}_{0}\right)$ for convention. By definition, $\tilde{Y}_{0}=c_{\mathfrak{g}} Y_{0}$. 
Lemma 7.1.1. There exists a system of vectors $\left(\left\{X_{i}\right\}_{i=0}^{n-1} ;\left\{Z_{i}\right\}_{i=0}^{n-1}\right)$ in $\mathfrak{g}_{\mathbf{C}}$ with the following properties.

(a) The vector $Z_{0}$ belongs to the center of $\mathfrak{k} \cap \mathfrak{h}$. The vectors $Z_{i}, 1 \leqslant i \leqslant n-1$ are in $(\mathfrak{k} \cap \mathfrak{q})_{\mathbf{C}}$. The vector $X_{0}$ and its complex conjugate $\bar{X}_{0}$ make up a $\mathbf{C}$ basis of $(\mathfrak{p} \cap \mathfrak{q})_{\mathbf{C}}$. The vectors $X_{i}, 1 \leqslant i \leqslant n-1$ together with their complex conjugates $\bar{X}_{i}$ make up a $\mathbf{C}$-basis of $(\mathfrak{p} \cap \mathfrak{h})_{\mathbf{C}}$. Moreover $\tilde{Y}_{0}=X_{0}+\bar{X}_{0}$.

(b) The vectors $X_{0}, \bar{X}_{0}$ and $-\sqrt{-1} Z_{0}$ form an $\mathfrak{s l}_{2}$-triple in $\mathfrak{g}_{\mathbf{C}}$, i.e.,

$$
\left[Z_{0}, X_{0}\right]=2 \sqrt{-1} X_{0},\left[Z_{0}, \bar{X}_{0}\right]=-2 \sqrt{-1} \bar{X}_{0},\left[X_{0}, \bar{X}_{0}\right]=-\sqrt{-1} Z_{0} .
$$

(c) When $(\mathfrak{g}, \mathfrak{h})$ is of type $(\mathrm{U})$, for $1 \leqslant i \leqslant n-1$

$$
\begin{aligned}
& \operatorname{ad}\left(Z_{0}\right) X_{i}=\sqrt{-1} X_{i}, \quad \operatorname{ad}\left(Z_{0}\right) Z_{i}=-\sqrt{-1} Z_{i}, \\
& \operatorname{ad}\left(X_{0}\right) X_{i}=0, \quad \operatorname{ad}\left(X_{0}\right) Z_{i}=-X_{i}, \\
& \operatorname{ad}\left(\bar{X}_{0}\right) X_{i}=-Z_{i}, \quad \operatorname{ad}\left(\bar{X}_{0}\right) Z_{i}=0 .
\end{aligned}
$$

When $(\mathfrak{g}, \mathfrak{h})$ is of type $(\mathrm{O})$, for $1 \leqslant i \leqslant n-1 Z_{i}=\bar{Z}_{i}$ and

$$
\begin{aligned}
& \operatorname{ad}\left(Z_{0}\right) Z_{i}=0, \operatorname{ad}\left(Z_{0}\right) X_{i}=2 \sqrt{-1} X_{i}, \operatorname{ad}\left(Z_{0}\right) \bar{X}_{i}=-2 \sqrt{-1} \bar{X}_{i}, \\
& \operatorname{ad}\left(X_{0}\right) Z_{i}=-X_{i}, \operatorname{ad}\left(X_{0}\right) X_{i}=0, \operatorname{ad}\left(X_{0}\right) \bar{X}_{i}=-2 Z_{i}, \\
& \operatorname{ad}\left(\bar{X}_{0}\right) Z_{i}=-\bar{X}_{i}, \operatorname{ad}\left(\bar{X}_{0}\right) X_{i}=-2 Z_{i}, \operatorname{ad}\left(\bar{X}_{0}\right) \bar{X}_{i}=0 .
\end{aligned}
$$

(d) When $(\mathfrak{g}, \mathfrak{h})$ is of type $(\mathrm{U})$, for $1 \leqslant i, j \leqslant n-1$

$$
\begin{array}{lll}
{\left[Z_{i}, X_{j}\right]=0,} & {\left[\bar{Z}_{i}, X_{j}\right]=-\delta_{i j} X_{0},} \\
{\left[\bar{Z}_{i}, \bar{X}_{j}\right]=0,} & {\left[Z_{i}, \bar{X}_{j}\right]=-\delta_{i j} \bar{X}_{0} .}
\end{array}
$$

When $(\mathfrak{g}, \mathfrak{h})$ is of type $(\mathrm{O})$, for $1 \leqslant i, j \leqslant n-1$

$$
\left[Z_{i}, X_{j}\right]=-\delta_{i j} X_{0}, \quad\left[Z_{i}, \bar{X}_{j}\right]=-\delta_{i j} \bar{X}_{0}
$$

(e) The values of the Killing form on $X_{i}, \bar{X}_{j}$ is given by

$$
\begin{array}{ll}
B\left(X_{i}, X_{j}\right)=B\left(\bar{X}_{i}, \bar{X}_{j}\right)=0, & 0 \leqslant i, j \leqslant n-1, \\
B\left(X_{i}, \bar{X}_{j}\right)=\frac{1}{2} \delta_{i j} B\left(\tilde{Y}_{0}, \tilde{Y}_{0}\right), & 0 \leqslant i, j \leqslant n-1 .
\end{array}
$$


(f) (Cartan-Iwasawa decomposition) Put $a_{t}=\exp \left(t Y_{0}\right)$ for $t \in \mathbf{R}$. When $(\mathfrak{g}, \mathfrak{h})$ is of type $(\mathrm{U})$, for $1 \leqslant i \leqslant n-1$ and $t \in \mathbf{R}-\{0\}$ the formulas

$$
\begin{aligned}
X_{0}= & -\frac{\sqrt{-1}}{4 \sinh (t) \cosh (t)} \operatorname{Ad}\left(a_{t}^{-1}\right) Z_{0}+\frac{1}{2} Y_{0} \\
& +\frac{\sqrt{-1}}{4}(\tanh (t)+\operatorname{coth}(t)) Z_{0}, \\
X_{i}= & \frac{1}{\cosh (t)} \operatorname{Ad}\left(a_{t}^{-1}\right) X_{i}-\tanh (t) Z_{i}
\end{aligned}
$$

hold. When $(\mathfrak{g}, \mathfrak{h})$ is of type $(\mathrm{O})$, for $1 \leqslant i \leqslant n-1$ and $t \in \mathbf{R}-\{0\}$ the formulas

$$
\begin{aligned}
X_{0}= & -\frac{\sqrt{-1}}{2 \sinh (t)} \operatorname{Ad}\left(a_{t}^{-1}\right) Z_{0}+Y_{0}+\frac{\sqrt{-1}}{\operatorname{coth}(t)} Z_{0}, \\
X_{i}= & \frac{1}{2}\left(1+\frac{1}{\cosh (t)}\right) \operatorname{Ad}\left(a_{t}^{-1}\right) X_{i} \\
& -\frac{1}{2}\left(1-\frac{1}{\cosh (t)}\right) \operatorname{Ad}\left(a_{t}^{-1}\right) \bar{X}_{i}-\tanh (t) Z_{i},
\end{aligned}
$$

hold.

Proof. Let $(\mathfrak{g}, \mathfrak{h})$ be of type (U). We may assume

$$
\mathfrak{g}=\left\{\left.X \in \mathfrak{s l}_{n+1}(\mathbf{C})\right|^{t} \bar{X} I_{n, 1}+I_{n, 1} X=O_{n+1}\right\}
$$

and take the involutions $\theta$ and $\sigma$ such that $\theta(X)=\left(-\bar{x}_{j i}\right)$ and $\sigma(X)=S_{\mathrm{U}} X S_{\mathrm{U}}^{-1}$ with $S_{\mathrm{U}}=\operatorname{diag}\left(E_{n-1},-1,1\right)$ for $X=\left(x_{i j}\right) \in \mathfrak{g}$. Here $E_{i}$ denotes the identity matrix of size $i$ and $I_{i, j}=\operatorname{diag}\left(E_{i},-E_{j}\right)$. We naturally identify $\mathfrak{g}_{\mathbf{C}}$ with $\mathfrak{g l}_{n+1}(\mathbf{C})$, which has a basis consisting of matrix units $E_{i, j}=\left(\delta_{i u} \delta_{j v}\right)_{u, v}, 1 \leqslant$ $i, j \leqslant n+1$. Now we take the vector $Y_{0}=E_{n, n+1}+E_{n+1, n}$ and put

$$
\begin{aligned}
& Z_{0}=\sqrt{-1}\left(E_{n, n}-E_{n+1, n+1}\right), \quad Z_{i}=E_{i, n}, \\
& X_{0}=E_{n+1, n}, \quad X_{i}=E_{i, n+1}
\end{aligned}
$$

for $1 \leqslant i \leqslant n-1$. Then direct computation shows the formulas in lemma. Let $(\mathfrak{g}, \mathfrak{h})$ be of type $(\mathrm{O})$. We may realize $\mathfrak{g}$ as

$$
\mathfrak{g}=\left\{\left.X \in \mathfrak{s l}_{n+2}(\mathbf{R})\right|^{t} X I_{n, 2}+I_{n, 2} X=O_{n+2}\right\}
$$

and take the involutions $\theta$ and $\sigma$ as $\theta(X)=-{ }^{t} X$ and $\sigma(X)=S_{\mathrm{O}} X S_{\mathrm{O}}^{-1}$ with $S_{\mathrm{O}}=\operatorname{diag}\left(E_{n-1},-1, E_{2}\right)$ for $X \in \mathfrak{g}$. Now we take $Y_{0}=E_{n, n+1}+E_{n+1, n} \in \mathfrak{g}$ 
and put

$$
\begin{aligned}
Z_{0} & =2\left(E_{n+1, n+2}-E_{n+2, n+1}\right), \\
Z_{i} & =E_{i, n}-E_{n, i}, \\
X_{0} & =E_{n, n+1}+E_{n+1, n}+\sqrt{-1}\left(E_{n, n+2}+E_{n+2, n}\right), \\
X_{i} & =E_{i, n+1}+E_{n+1, i}+\sqrt{-1}\left(E_{i, n+2}+E_{n+2, i}\right)
\end{aligned}
$$

for $1 \leqslant i \leqslant n-1$. Then by direct computation the formulas in lemma follow.

We give a formula which describes the radial part of the action of the element $X_{i} \bar{X}_{j}$ to a vector-valued function on $U=G_{\mathbf{R}}-S$ with $S=H_{\mathbf{R}} K$. Let $R$ be the representation of $U\left(\mathfrak{g}_{\mathbf{C}}\right)$ on the space of those functions $\varphi$ defined by $R_{X} \varphi=\varphi * X$ for $X \in U\left(\mathfrak{g}_{\mathbf{C}}\right)$.

Lemma 7.1.2. Let $(\tau, V)$ be a unitary representation of $K$. Let $\varphi$ : $G_{\mathbf{R}}-S \rightarrow V$ be a $C^{\infty}$ function such that

$$
\varphi(h g k)=\tau(k)^{-1} \varphi(g), \quad h \in H_{\mathbf{R}}, g \in G_{\mathbf{R}}-S, k \in K .
$$

Put $\phi(t)=\varphi\left(a_{t}\right)$ for $t \in \mathbf{R}-\{0\}$.

(1) When $(\mathfrak{g}, \mathfrak{h})$ is of type $(\mathrm{U})$, we have

$$
\begin{aligned}
\left(R_{X_{j} \bar{X}_{i}} \varphi\right)\left(a_{t}\right)= & \left(\frac{\delta_{i j}}{2} \tanh (t) \frac{d}{d t}+\frac{\sqrt{-1}}{4} \delta_{i j}\left(\tanh ^{2}(t)+1\right) \tau\left(Z_{0}\right)\right. \\
& \left.+\tau\left(Z_{j} \bar{Z}_{i}\right) \tanh ^{2}(t)\right) \phi(t) \\
\left(R_{X_{j} \bar{X}_{0}} \varphi\right)\left(a_{t}\right)= & \frac{1}{2} \tau\left(Z_{j}\right)\left(\tanh (t) \frac{d}{d t}+\frac{\sqrt{-1}}{2}\left(\tanh ^{2}(t)+1\right) \tau\left(Z_{0}\right)\right) \phi(t), \\
\left(R_{X_{0} \bar{X}_{i}} \varphi\right)\left(a_{t}\right)= & \frac{1}{2} \tau\left(\bar{Z}_{i}\right)\left(\tanh (t) \frac{d}{d t}+2-\frac{\sqrt{-1}}{2}\left(\tanh ^{2}(t)+1\right) \tau\left(Z_{0}\right)\right) \phi(t), \\
\left(R_{X_{0} \bar{X}_{0}} \varphi\right)\left(a_{t}\right)= & \frac{1}{4}\left(\frac{d^{2}}{d t^{2}}+(\tanh (t)+\operatorname{coth}(t)) \frac{d}{d t}\right. \\
& \left.+2 \sqrt{-1} \tau\left(Z_{0}\right)+\frac{1}{4}(\tanh (t)+\operatorname{coth}(t))^{2} \tau\left(Z_{0}^{2}\right)\right) \phi(t),
\end{aligned}
$$

for $t \in \mathbf{R}-\{0\}$ and $1 \leqslant i, j \leqslant n-1$.

(2) When $(\mathfrak{g}, \mathfrak{h})$ is of type $(\mathrm{O})$, we have

$$
\left(R_{X_{j} \bar{X}_{i}} \varphi\right)\left(a_{t}\right)=\left(\delta_{i j} \tanh (t) \frac{d}{d t}+\tanh ^{2}(t) \tau\left(Z_{j} Z_{i}\right)+\frac{\sqrt{-1}}{2} \delta_{i j} \tau\left(Z_{0}\right)\right) \phi(t),
$$




$$
\begin{aligned}
& \left(R_{X_{0} \bar{X}_{i}} \varphi\right)\left(a_{t}\right)=\tau\left(Z_{i}\right)\left(\tanh (t) \frac{d}{d t}+\frac{\sqrt{-1}}{2} \tau\left(Z_{0}\right)+\frac{1}{\cosh ^{2}(t)}+1\right) \phi(t), \\
& \left(R_{X_{j} \bar{X}_{0}} \varphi\right)\left(a_{t}\right)=\tau\left(Z_{j}\right)\left(\tanh (t) \frac{d}{d t}+\frac{\sqrt{-1}}{2} \tau\left(Z_{0}\right)-\tanh ^{2}(t)\right) \phi(t), \\
& \left(R_{X_{0} \bar{X}_{0}} \varphi\right)\left(a_{t}\right)=\left(\frac{d^{2}}{d t^{2}}+\operatorname{coth}(t) \frac{d}{d t}+\frac{\sqrt{-1}}{2} \tau\left(Z_{0}\right)+\frac{1}{4} \operatorname{coth}^{2}(t) \tau\left(Z_{0}^{2}\right)\right) \phi(t),
\end{aligned}
$$

for $t \in \mathbf{R}-\{0\}$ and $1 \leqslant i, j \leqslant n-1$.

Proof. We only give a proof of the first formula in (1). The remaining cases are very similar. First by the second formula (7.1.12), secondly since $R_{\bar{X}_{i}} \varphi$ is left $H_{\mathbf{R}}$-invariant, after that by $(7.1 .10)$, we have

$$
\begin{aligned}
R_{X_{j} \bar{X}_{i}} \varphi\left(a_{t}\right) & =R_{X_{j}}\left(R_{\bar{X}_{i}} \varphi\right)\left(a_{t}\right) \\
& =-\tanh (t) R_{Z_{j}}\left(R_{\bar{X}_{i}} \varphi\right)\left(a_{t}\right) \\
& =-\tanh (t)\left(R_{\bar{X}_{i}} R_{Z_{j}}+R_{\left[Z_{j}, \bar{X}_{i}\right]}\right) \varphi\left(a_{t}\right) \\
& =-\tanh (t)\left(-\tau\left(Z_{j}\right) R_{\bar{X}_{i}} \varphi\left(a_{t}\right)-\delta_{i j} R_{\bar{X}_{0}} \varphi\left(a_{t}\right)\right) .
\end{aligned}
$$

From (7.1.12), we have

$$
\begin{aligned}
R_{\bar{X}_{i}} \varphi\left(a_{t}\right) & =-\tanh (t) R_{\bar{Z}_{i}} \varphi\left(a_{t}\right)=\tanh (t) \tau\left(\bar{Z}_{i}\right) \phi(t) \\
R_{\bar{X}_{0}} \varphi\left(a_{t}\right) & =\frac{1}{2} R_{Y_{0}} \varphi(t)-\frac{\sqrt{-1}}{4}(\tanh (t)+\operatorname{coth}(t)) R_{Z_{0}} \varphi\left(a_{t}\right) \\
& =\frac{1}{2}\left(\frac{d}{d t}+\frac{\sqrt{-1}}{2}(\tanh (t)+\operatorname{coth}(t)) \tau\left(Z_{0}\right)\right) \phi(t) .
\end{aligned}
$$

From (7.1.14) and (7.1.15), we obtain the first formula in (1).

\section{$\S 7.2$. Space of differential forms}

Since $\mathfrak{g} \cong \mathfrak{s o}(n, 2)$ or $\mathfrak{s u}(n, 1)$, the homogeneous manifold $\mathfrak{D}=G_{\mathbf{R}} / K$ has a $G_{\mathbf{R}}$-invariant complex structure coming from the adjoint action on $\mathfrak{p}$ of a central element of $\mathfrak{k}$. Firstly we fix the complex structure explicitly.

Lemma 7.2.1. If $(\mathfrak{g}, \mathfrak{h})$ is of type $(\mathrm{U})$, we put

$$
\tilde{Z}_{0}=\frac{1}{n+1}\left(n Z_{0}-\sqrt{-1} \sum_{i=1}^{n-1}\left[Z_{i}, \bar{Z}_{i}\right]\right)
$$

if $(\mathfrak{g}, \mathfrak{h})$ is of type $(\mathrm{O})$, we put $\tilde{Z}_{0}=2^{-1} Z_{0}$. Then the center of $\mathfrak{k}$ is generated by the element $\tilde{Z}_{0}$. The eigenvalue of $\operatorname{ad}\left(\tilde{Z}_{0}\right)$ on $\mathfrak{p}_{\mathbf{C}}$ is $\pm \sqrt{-1}$. Let $\mathfrak{p}_{+}$be the $\mathbf{C}$-span of $X_{i}$ 's with $0 \leqslant i \leqslant n-1$ and $\mathfrak{p}_{-}$the complex conjugate of $\mathfrak{p}_{+}$. These are the $\epsilon \sqrt{-1}$-eigenspace of $\operatorname{ad}\left(\tilde{Z}_{0}\right) \mid \mathfrak{p}_{\mathbf{C}}$ for $\epsilon \in\{+,-\}$ respectively. 
Proof. By Lemma 7.1.1, we have $\mathfrak{p}_{\mathbf{C}}=\mathfrak{p}_{+} \oplus \mathfrak{p}_{-}$. We prove the last statement. Assume $(\mathfrak{g}, \mathfrak{h})$ is of type $(\mathrm{O})$. Then the desired statement is a consequence of the formulas in (7.1.1) and (7.1.5). Assume $(\mathfrak{g}, \mathfrak{h})$ is of type (U). By the formulas in (7.1.9), we have $\left[\left[Z_{i}, \bar{Z}_{i}\right], X_{j}\right]=-\delta_{i j} X_{j}$ for $1 \leqslant i, j \leqslant n-1$ after a computation. From this equation, we obtain $\left[\tilde{Z}_{0}, X_{j}\right]=\sqrt{-1} X_{j}$ for $1 \leqslant j \leqslant n-1$ easily. Similarly we can prove $\left[\tilde{Z}_{0}, X_{0}\right]=\sqrt{-1} X_{0}$. Thus the last statement follows. Since $\operatorname{ad}\left(Z_{0}\right) \mid \mathfrak{p}_{+}$is a scalar operator, $\operatorname{ad}\left(Z_{0}\right) \mid \mathfrak{p}_{+}$is commutative with $\operatorname{ad}(Z) \mid \mathfrak{p}_{+}$for any $Z \in \mathfrak{k}$. Now the assumption $\mathfrak{g} \cong \mathfrak{s o}(n, 2)$, or $\mathfrak{s u}(n, 1)$ implies that the representation ad $: \mathfrak{k} \rightarrow \operatorname{End}_{\mathbf{C}}\left(\mathfrak{p}_{+}\right)$is faithfull. Hence $\left[\tilde{Z}_{0}, Z\right]=0$ for $Z \in \mathfrak{k}$.

We take the complex structure of $\mathfrak{D}$ determined by $I=\operatorname{ad}\left(\tilde{Z}_{0}\right) \mid \mathfrak{p}$. Similarly $\mathfrak{D}_{H}=H_{\mathbf{R}} / H_{\mathbf{R}} \cap K$ becomes a complex manifold endowed with the $H_{\mathbf{R}^{-}}$ invariant complex structure determined by $I_{o}=\operatorname{ad}\left(c_{\mathfrak{g}}^{-1} Z_{0}\right) \mid(\mathfrak{p} \cap \mathfrak{h})$. Then the embedding $\mathfrak{D}_{H} \hookrightarrow \mathfrak{D}$ is holomrphic. Let $A^{p, q}(\mathfrak{D})$ be the space of $C^{\infty}$ differential forms of type $(p, q)$ on the complex manifold $\mathfrak{D}$. The group $G_{\mathbf{R}}$ acts on $A^{p, q}(\mathfrak{D})$ naturally since $\mathfrak{D}$ is a $G_{\mathbf{R}}$-space. Then as usual, we can naturally identify the de Rham complex $A(\mathfrak{D})=\oplus_{p, q} A^{p, q}(\mathfrak{D})$ of $\mathfrak{D}$ with the $(\mathfrak{g}, K)$-complex $C\left(\mathfrak{g}, K ; C^{\infty}\left(G_{\mathbf{R}}\right)\right)$. Here we regard $C^{\infty}\left(G_{\mathbf{R}}\right)$ as a $(\mathfrak{g}, K)$-module via the right action $R$ of $\mathfrak{g}$ and $K$. See [2, Chapter VII]. For a unitary representation $(\tau, V)$ of $K$ and an open subset $U$ of $G_{\mathbf{R}}$ such that $U K=U$, let $C^{\infty}(U ; \tau)$ be the space of those $C^{\infty}$-functions $\varphi: G_{\mathbf{R}} \rightarrow V$ such that

$$
\varphi(g k)=\tau(k)^{-1} \varphi(g), \quad(g, k) \in U \times K .
$$

Put $\sigma_{p q}=\wedge^{p} \mathrm{Ad}_{+} \otimes \wedge^{q} \mathrm{Ad}_{-}$. Then we have a natural identification preserving the (left) $G_{\mathbf{R}}$-actions

$$
A^{p, q}(\mathfrak{D}) \cong C^{p, q}\left(\mathfrak{g}, K ; C^{\infty}\left(G_{\mathbf{R}}\right)\right) \cong C^{\infty}\left(G_{\mathbf{R}} ; \sigma_{p q}^{*}\right) .
$$

For a form $\omega \in A(\mathfrak{D})$, the corresponding function in $C^{\infty}\left(G_{\mathbf{R}} ; \sigma_{p q}^{*}\right)$ will be denoted by $\tilde{\omega}$. We also have the counterparts for $H$ of $A(\mathfrak{D}), C^{\infty}\left(G_{\mathbf{R}} ; \sigma_{p q}^{*}\right)$ and the isomorphism (7.2.1). The $K$-invariant hermitian inner product of the complex vector space $\mathfrak{p}$

$$
\langle X, Y\rangle=B\left(\tilde{Y}_{0}, \tilde{Y}_{0}\right)^{-1}(B(X, Y)+\sqrt{-1} B(X, I Y)), \quad X, Y \in \mathfrak{p}
$$

defines a $G_{\mathbf{R}}$-invariant hermitian metric on the tangent bundle of $\mathfrak{D}$. This is a Kähler metric with the Kähler form $\omega_{\mathfrak{D}}$ such that

$$
\tilde{\omega}_{\mathfrak{D}}=\frac{\sqrt{-1}}{2} \sum_{i=0}^{n-1} \omega_{i} \wedge \bar{\omega}_{i}
$$


with $\left\{\omega_{i}, \bar{\omega}_{j}\right\}$ the dual basis of $\left\{X_{i}, \bar{X}_{j}\right\}$. Let $v_{\mathfrak{D}}=(n !)^{-1} \omega_{\mathfrak{D}}^{n}$ be the volume form of $\mathfrak{D}$. Similarly, $H_{\mathbf{R}} \cap K$-invariant inner product $\langle\rangle \mid,(\mathfrak{p} \cap \mathfrak{h})$ determines an $H_{\mathbf{R}}$-invariant Kähler structure on $\mathfrak{D}_{H}$. Let $\omega_{\mathfrak{D}_{H}}$ and $v_{\mathfrak{D}_{H}}$ be the Kähler form and the volume form respectively.

Lemma 7.2.2. $\quad$ Let $d k$ be the normalized Haar measure of the compact group $K$. Let $d v(g)$ be the Haar measure of $G_{\mathbf{R}}$ such that the quotient measure of $d v(g)$ by $d k$ corresponds to the measure of $\mathfrak{D}$ determined by $v_{\mathfrak{D}}$. Similarly we take the normalized Haar measure dh of $H_{\mathbf{R}}$ corresponding to $v_{\mathfrak{D}_{H}}$. Then $d v(g)$ equals $2 \pi c_{\mathfrak{g}}^{-2}$ times the measure $d g$ determined from dh by the formula (1.3.2).

Proof. By calculating the Jacobian determinant associated with the decomposition $\mathfrak{g}=\operatorname{Ad}\left(a_{t}\right) \mathfrak{h}+\mathbf{R} Y_{0}+\mathfrak{k}$ with the aid of the formulas in Lemma 7.2.1 and by examining $\operatorname{vol}(H \cap K / M)$, we have the conclusion. We omit the detail.

Let $\Gamma$ be a neat arithmetic subgroup of $G_{\mathbf{Q}}$. In this case the double coset space $\Gamma \backslash \mathfrak{D}=\Gamma \backslash G_{\mathbf{R}} / K$ is naturally a complex manifold in such a way that the quotient map $\pi: \mathfrak{D} \rightarrow \Gamma \backslash \mathfrak{D}$ becomes a local isomorphism of complex manifolds. The Kähler metric of $\mathfrak{D}$ is pushed down to $\Gamma \backslash \mathfrak{D}$ giving rise to a Kähler metric of $\Gamma \backslash \mathfrak{D}$. Let $\omega_{\Gamma \backslash \mathfrak{D}}$ and $v_{\Gamma \backslash \mathfrak{D}}$ be the Kähler form and the volume form of $\Gamma \backslash \mathfrak{D}$ respectively. Analogously the space $\Gamma_{H} \backslash \mathfrak{D}_{H}$ with $\Gamma_{H}=\Gamma \cap H_{\mathbf{R}}$ becomes a Kähler manifold. The Kähler form and the volume form of $\Gamma_{H} \backslash \mathfrak{D}_{H}$ are denoted by $\omega_{\Gamma_{H} \backslash \mathfrak{D}_{H}}$ and $v_{\Gamma_{H} \backslash \mathfrak{D}_{H}}$ respectively.

Through the pullback via the quotient map $\pi$, the de Rham complex $A(\Gamma \backslash \mathfrak{D})$ of $\Gamma \backslash \mathfrak{D}$ is naturally identified with the $\Gamma$-invariant part of $A(\mathfrak{D})$ preserving the bidegree. For $\omega \in A(\Gamma \backslash \mathfrak{D})$ the corresponding function $\tilde{\omega}$ is left $\Gamma$-invariant.

Let $\partial^{*}$ and $\bar{\partial}^{*}$ be the adjoint of the holomorphic exterior derivative $\partial$ and the antiholomorphic exterior derivative $\bar{\partial}$ on $\Gamma \backslash \mathfrak{D}$ respectively. Recall that they satisfy

$$
\partial^{*} \omega=(-1)^{d} *^{-1} \bar{\partial} * \omega, \quad \bar{\partial}^{*} \omega=(-1)^{d} *^{-1} \partial * \omega
$$

for any $d$-form $\omega$ on $\Gamma \backslash \mathfrak{D}$. Here $*$ denotes the Hodge $*$-operator, which is an operator on $A(\Gamma \backslash \mathfrak{D})$ which sends a $(p, q)$-form to a $(n-q, n-p)$-form. The Laplacian on forms is defined by $\triangle=d d^{*}+d^{*} d$ with $d=\partial+\bar{\partial}$ and $d^{*}=\partial^{*}+\bar{\partial}^{*}$. 
Proposition 7.2.1.

(1) We have

$$
\begin{aligned}
& *\left(\omega_{i} \wedge \bar{\omega}_{i}\right)=\left(\frac{\sqrt{-1}}{2}\right)^{n-2} \prod_{k \neq i} \omega_{k} \wedge \bar{\omega}_{k}, \\
& *\left(\omega_{i} \wedge \bar{\omega}_{j}\right)=-\left(\frac{\sqrt{-1}}{2}\right)^{n-2} \omega_{i} \wedge \bar{\omega}_{j} \wedge \prod_{k \neq i, j} \omega_{k} \wedge \bar{\omega}_{k}
\end{aligned}
$$

for $0 \leqslant i, j \leqslant n-1$ with $i \neq j$.

(2) Given an $\omega \in A^{1,1}(\Gamma \backslash \mathfrak{D})$ we have

$$
\begin{aligned}
\frac{1}{4}\left(\bar{\partial}^{*} \partial^{*} \omega\right)^{\sim}(g)= & \sum_{i, j=0}^{n-1}\left\langle X_{i} \wedge \bar{X}_{j}, R_{X_{j} \bar{X}_{i}} \tilde{\omega}(g)\right\rangle, \\
\frac{1}{2}\left\langle X_{i} \wedge \bar{X}_{j},(\triangle \omega)^{\sim}\right\rangle(g)= & -\sum_{h=0}^{n-1}\left\langle X_{i} \wedge \bar{X}_{j},\left(R_{\bar{X}_{h} X_{h}}+R_{X_{h} \bar{X}_{h}}\right) \tilde{\omega}(g)\right\rangle \\
& -\sum_{h=1}^{n-1}\left\langle X_{i} \wedge \bar{X}_{j},\left(R_{\left[X_{i}, \bar{X}_{h}\right]}+R_{\left[\bar{X}_{j}, X_{h}\right]}\right) \tilde{\omega}(g)\right\rangle .
\end{aligned}
$$

(3) Given a $\varphi \in A^{0,0}(\Gamma \backslash \mathfrak{D})$, we have

$$
(\triangle \varphi) \sim(g)=-c_{\mathfrak{g}}^{2} R_{\Omega} \tilde{\varphi}(g),
$$

where $\Omega$ is the renormalized Casimir element introduced in 2.1.

Proof. (1) See [35, p. 19]. (2), (3) See [2, Chapter II].

\section{$\S 7.3$. Currents associated with the modular divisor}

Recall that $D_{o}$ is the image of the natural holomorphic map

$$
j: \Gamma_{H} \backslash \mathfrak{D}_{H} \rightarrow \Gamma \backslash \mathfrak{D},
$$

where $\Gamma_{H}=\Gamma \cap H_{\mathbf{R}}$. We introduce the $(0,0)$-current $* \delta_{D_{o}}$ on $\Gamma \backslash \mathfrak{D}$ by

$$
\left\langle * \delta_{D_{o}}, \varphi\right\rangle=\int_{\Gamma_{H} \backslash \mathfrak{D}_{H}} j^{*}(* \varphi) \cdot v_{\Gamma_{H} \backslash \mathfrak{D}_{H}}, \quad \varphi \in A_{\mathrm{c}}^{n, n}(\Gamma \backslash \mathfrak{D}) .
$$

We also define the $(1,1)$-current $\tilde{\delta}_{D_{o}}$ on $\Gamma \backslash \mathfrak{D}$ by

$$
\left\langle\tilde{\delta}_{D_{o}}, \eta\right\rangle=\int_{\Gamma_{H} \backslash \mathfrak{D}_{H}} j^{*}(\eta), \quad \eta \in A_{\mathrm{c}}^{n-1, n-1}(\Gamma \backslash \mathfrak{D}) .
$$




\section{$\S 7.4$. $\quad K$-module corresponding to the space of $(1,1)$-forms}

The space $\mathfrak{p}_{ \pm}$is a $K$-stable subspace of $\mathfrak{p}_{\mathbf{C}}$. Let $\operatorname{Ad}_{\epsilon}$ be the action of $K$ on the space $\mathfrak{p}_{\epsilon}$ for $\epsilon \in\{+,-\}$.

Since the Killing form $B: \mathfrak{p}_{-} \times \mathfrak{p}_{+} \rightarrow \mathbf{C}$ gives a perfect pairing (see Lemma 7.1.1), the C-bilinear form $\langle$,$\rangle on \mathfrak{p}_{-} \wedge \mathfrak{p}_{+}$such that

$$
\left\langle X \wedge Y, X^{\prime} \wedge Y^{\prime}\right\rangle=B\left(\tilde{Y}_{0}, \tilde{Y}_{0}\right)^{-2} B\left(X, Y^{\prime}\right) \cdot B\left(X^{\prime}, Y\right), \quad X, X^{\prime} \in \mathfrak{p}_{-}, Y, Y^{\prime} \in \mathfrak{p}_{+}
$$

is also non-degenerate.

We need an irreducible decomposition of the tensor product $\mathrm{Ad}_{-} \otimes \mathrm{Ad}_{+}$ and the structure of the $M$-invariant part of $\mathfrak{p}_{-} \otimes \mathfrak{p}_{+}$. Here $M$ denotes the centralizer of $\mathfrak{a}_{\mathfrak{p}, \mathfrak{q}}=\mathbf{R} Y_{0}$ in $H_{\mathbf{R}} \cap K$.

\section{Lemma 7.4.1.}

(1) The representation $\mathrm{Ad}_{-} \otimes \mathrm{Ad}_{+}$is self-adjoint and has two $M$-spherical irreducible components. Put

$$
v_{0}=\sum_{i=0}^{n} \bar{X}_{i} \wedge X_{i}
$$

Then $v_{0}$ is a nonzero $K$-invariant tensor in $\mathfrak{p}_{-} \otimes \mathfrak{p}_{+}$. Let $V_{11}$ be the orthogonal complement of $\mathbf{C} v_{0}$ in $\mathfrak{p}_{-} \otimes \mathfrak{p}_{+}$. Then $V_{11}$ is a $K$-invariant subspace of $\mathfrak{p}_{-} \otimes \mathfrak{p}_{+}$.

(2) The $M$-invariant part $V_{11}^{M}$ is one dimensional. Put

$$
v_{11}=\sum_{i=1}^{n-1} \bar{X}_{i} \wedge X_{i}-(n-1) \bar{X}_{0} \wedge X_{0} .
$$

Then the tensor $v_{11}$ gives a nonzero element of $V_{11}^{M}$.

Proof. (1) The self-duality of $\mathrm{Ad}_{-} \otimes \mathrm{Ad}_{+}$is a consequence of the fact that the pairing $\langle$,$\rangle is K$-invariant. Since $v_{0}$ corresponds to the $K$-invariant bilinear form $B \mid \mathfrak{p}_{+} \times \mathfrak{p}_{-}$, it is $K$-invariant. By a similar reason, the tensor $\sum_{i=1}^{n-1} \bar{X}_{i} \wedge X_{i}$ is $H_{\mathbf{R}} \cap K$-invariant. Put $2 E_{0}=Z_{0}+\sqrt{-1}\left(\bar{X}_{0}-X_{0}\right)$ and $2 F_{0}=-Z_{0}+\sqrt{-1}\left(\bar{X}_{0}-X_{0}\right)$. Then using Lemma 7.1.1 (a) and the formula (7.1.1), one can prove that $\mathfrak{g}_{\lambda_{0}}^{\sigma \theta}=\mathbf{R} E_{0}$, on which the compact group $M$ acts by adjoint. Hence for an $m \in M$ we have $\operatorname{Ad}(m) E_{0}=\epsilon E_{0}$ with $\epsilon \in\{+,-\}$. Applying $-\theta$ to the last equation, we have $\operatorname{Ad}(m) F_{0}=\epsilon F_{0}$. Hence $\operatorname{Ad}(m) X_{0}$ equals $X_{0}$ or $\bar{X}_{0}$ according as $\epsilon=+$ or $\epsilon=-$. Since $\operatorname{Ad}(m)$ preserves $\mathfrak{p}_{\epsilon}$, we 
must have $\operatorname{Ad}(m) X_{0}=X_{0}$. Similarly we have $\operatorname{Ad}(m) \bar{X}_{0}=\bar{X}_{0}$. Hence the tensor $\bar{X}_{0} \wedge X_{0}$ is fixed by $M$. It is obvious that $v_{0}$ and $v_{11}$ are orthogonal. Thus we have $v_{11} \in V_{11}^{M}$. The pair $(\mathfrak{k}, \mathfrak{m})$ is, up to an abelian factor, isomorphic to $(\mathfrak{u}(n), \mathfrak{u}(n-1))$ or $(\mathfrak{s o}(n), \mathfrak{s o}(n-1))$. Hence by a case study it turns out that the $K$-module $V_{11}$ containes exactly one irreducible $M$-spherical constituent and $\operatorname{dim} V_{11}^{M}=1$.

The action of $K$ on $V_{11}$ will be denoted by $\tau_{11}$.

\section{§7.5. A vector-valued Poincaré series}

Let $s \in \mathbf{C}$ with $\operatorname{Re}(s)>\rho_{0}$. Note that $\rho_{0}$ equals $c_{\mathfrak{g}}^{-1} n$. Let $\phi_{s}^{(2)}$ be the secondary spherical function defined in 2.4. Recall that it is a right $K$-invariant, left $H_{\mathbf{R}}$-invariant, C-valued $C^{\infty}$ function on the open set $U=G_{\mathbf{R}}-H_{\mathbf{R}} K$ with the radial part given by the formula (2.5.3).

For $\epsilon \in\{+,-\}$, let $\nabla_{\epsilon}$ be the Schmid operator ([30]). Recall that it is a first order elliptic differential operator which sends a function in $C^{\infty}(U ; \tau)$ to a function in $C^{\infty}\left(U ; \tau \otimes \operatorname{Ad}_{\epsilon}\right)$, where $U$ is an open subset of $G$ with $U K=U$, and $\tau$ is a unitary representation of $K$. In terms of the basis $\left\{X_{i}\right\}$ of $\mathfrak{p}_{+}$and $\left\{\bar{X}_{i}\right\}$ of $\mathfrak{p}_{-}$, we have

$$
\nabla_{+} \varphi(g)=\sum_{i=0}^{n-1} R_{\bar{X}_{i}} \varphi(g) \bigotimes X_{i}, \quad \nabla_{-} \varphi(g)=\sum_{i=0}^{n-1} R_{X_{i}} \varphi(g) \otimes \bar{X}_{i}
$$

for $\varphi \in C^{\infty}(U / K ; \tau)$.

We have the radial part of the composite $\nabla_{-} \nabla_{+}$. From now on we identify $\mathfrak{p}_{+} \otimes \mathfrak{p}_{-}$and $\mathfrak{p}_{+} \wedge \mathfrak{p}_{-}$canonically.

Proposition 7.5.1. Let $\varphi \in C^{\infty}\left(U ; \tau_{0}\right)$ with $\tau_{0}$ the one dimensional trivial representation of $K$. Then we have

$$
\begin{aligned}
- & \left(\nabla_{-} \nabla_{+} \varphi\right)\left(a_{t}\right) \\
= & \frac{c_{\mathfrak{g}}}{2}\left\{\frac{1}{2 \rho_{0}} \mathcal{L} \varphi\left(a_{t}\right) \cdot v_{0}+\left(\tanh (t) \frac{d}{d t}-\frac{1}{2 \rho_{0}} \mathcal{L}\right) \varphi\left(a_{t}\right) \cdot v_{11}\right\}, \quad t \in \mathbf{R}-\{0\}
\end{aligned}
$$

with

$$
\mathcal{L}=\frac{d^{2}}{d t^{2}}+\left(\left(2 \rho_{0}-1\right) \tanh (t)+\operatorname{coth}(t)\right) \frac{d}{d t} .
$$

Here $v_{0}$ and $v_{11}$ are $M$-invariant tensors in $\mathfrak{p}_{+} \wedge \mathfrak{p}_{-}$defined in Lemma 7.4.1.

Proof. By definition, we have

$$
-\nabla_{-} \nabla_{+} \varphi\left(a_{t}\right)=\sum_{i, j=0}^{n-1} R_{X_{j} \bar{X}_{i}} \varphi\left(a_{t}\right) \cdot\left(\bar{X}_{j} \wedge X_{i}\right) .
$$


Substitute the expressions in Lemma 7.1.2 for $R_{X_{j} \bar{X}_{i}} \varphi\left(a_{t}\right)$ in the right-hand side of this formula. Then after some computation, we have the desired formula without difficulty.

Let pr $: \mathfrak{p}_{+} \wedge \mathfrak{p}_{-} \rightarrow V_{11}$ be the orthogonal projection. We define the function $\psi_{s}: G_{\mathbf{R}}-S \rightarrow V_{11}$ by

$$
\psi_{s}(g)=-\operatorname{pr}\left(\nabla_{-} \nabla_{+} \phi_{s}^{(2)}(g)\right), \quad g \in G_{\mathbf{R}}-S .
$$

By the $\left(H_{\mathbf{R}}, K\right)$-equivariance of $\psi_{s}$, the radial part $\psi_{s} \mid\left(A_{\mathfrak{p}, \mathfrak{q}}-\{e\}\right)$ determines $\psi_{s}$ completely. Here is a formula of the radial part.

\section{Corollary 7.5.1.}

(1) We have $\psi_{s}\left(a_{t}\right)=f_{s}(t) \cdot v_{11}, t \in \mathbf{R}-\{0\}$ with

$$
f_{s}(t)=\frac{c_{\mathfrak{g}}}{2}\left(\tanh (t) \frac{d}{d t}-\frac{s^{2}-\rho_{0}^{2}}{2 \rho_{0}}\right) \phi_{s}^{(2)}\left(a_{t}\right), \quad t \neq 0 .
$$

(2) The function $f_{s}$ satisfies the differential equation:

$$
\left(\mathcal{L}+\frac{4 \rho_{0}}{\cosh ^{2}(t)}\right) f_{s}(t)=\left(s^{2}-\rho_{0}^{2}\right) f_{s}(t), \quad t \neq 0 .
$$

Proof. This follows from Proposition 7.5.1 and the differential equation of $\phi_{s}^{(2)}\left(a_{t}\right)$.

Corollary 7.5.2. Let $s \in \mathbf{C}$ with $\operatorname{Re}(s)>\rho_{0}$.

(1) On the interval $[1, \infty)$ we have the estimates

$$
\left|f_{s}(t)\right| \prec e^{-\left(\operatorname{Re}(s)+\rho_{0}\right) t}, \quad\left|\frac{d f_{s}}{d t}(t)\right| \prec e^{-\left(\operatorname{Re}(s)+\rho_{0}\right) t}, \quad t \geqslant 1 .
$$

On the finite interval $(0,1]$ we have the estimate

$$
\left|f_{s}(t)\right| \prec \log (t), \quad 0<t \leqslant 1 .
$$

(2) We have

$$
\lim _{\epsilon \rightarrow+0} \epsilon \frac{d f_{s}}{d t}(\epsilon)=-\frac{c_{\mathfrak{g}}\left(s^{2}-\rho_{0}^{2}\right)}{4 \rho_{0}} .
$$

Proof. This follows from the formula of $f_{s}$ in Corollary 7.5.1 and the power series expression of $\phi_{s}^{(2)}\left(a_{t}\right)$ in Corollary 2.4.1. 
Remark 7.5.1.

(1) The $V_{11}$-valued function

$$
\psi_{\rho_{0}}(g)=\lim _{s \rightarrow \rho_{0}} \psi_{s}(g), \quad g \in G_{\mathbf{R}}-H_{\mathbf{R}} K
$$

extends to a left $H_{\mathbf{R}}$-invariant $C^{\infty}$-function on all of $G_{\mathbf{R}}$. It satisfies the differential equation

$$
\psi_{\rho_{0}} * \Omega=0
$$

(2) The functions $\left\langle v, \psi_{\rho_{0}}(g)\right\rangle$ with $v \in V_{11}$ generate an irreducible unitary representaion in $L^{2}\left(H_{\mathbf{R}} \backslash G_{\mathbf{R}}\right)$, which we denote by $\pi^{1,1}$.

Now let us introduce a Poincaré series associated with the function $\psi_{s}$ as

$$
\tilde{\Psi}_{s}(g)=\sum_{\gamma \in \Gamma_{H} \backslash \Gamma} \psi_{s}(\gamma g), \quad g \in G_{\mathbf{R}} .
$$

If $\operatorname{Re}(s)>\rho_{0}$, by the same way as in Proposition 3.1.1 using the estimate in Corollary 7.5.2 we can show that the series $\tilde{\Psi}_{s}(g)$ converges absolutely almost everywhere in $g$ to give a $V_{11}$-valued locally $L^{1}$-function on $G_{\mathbf{R}}$ with the $(\Gamma, K)$ equivariance

$$
\tilde{\Psi}_{s}(\gamma g k)=\tau_{11}(k)^{-1} \tilde{\Psi}_{s}(g), \quad \gamma \in \Gamma, k \in K .
$$

Since $V_{11} \hookrightarrow \mathfrak{p}_{-} \wedge \mathfrak{p}_{+} \cong \mathfrak{p}_{+}^{*} \wedge \mathfrak{p}_{-}^{*}$, it defines a locally integrable measurable $(1,1)$ form on $\Gamma \backslash \mathfrak{D}$, which we denote by $\Psi_{s}$. We shall regard $\Psi_{s}$ as a $(1,1)$-current on $\Gamma \backslash \mathfrak{D}$.

\section{$\S 7.6 . \quad$ Differential equations, the main statement}

The function $G_{s}$ on $\Gamma \backslash G_{\mathbf{R}}$ introduced in 3.1 is right $K$-invariant. Hence it naturally defines a $(0,0)$-current on $\Gamma \backslash \mathfrak{D}$, which we denote by the same letter $G_{s}$. Here is the main result of this section.

Theorem 7.6.1. Let $n$ be the complex dimension of $\Gamma \backslash \mathfrak{D}$. Given $s \in \mathbf{C}$ with $\operatorname{Re}(s)>c_{\mathfrak{g}}^{-1} n$, we have the differential equations:

$$
\begin{aligned}
& \partial \bar{\partial} G_{s}+\pi \sqrt{-1} \tilde{\delta}_{D_{o}}=\frac{-\sqrt{-1}}{2 n}\left(\left(c_{\mathfrak{g}} s\right)^{2}-n^{2}\right) G_{s} \wedge \omega_{\Gamma \backslash \mathfrak{D}}+4 \Psi_{s} \\
& \triangle \Psi_{s}=-\left(\left(c_{\mathfrak{g}} s\right)^{2}-n^{2}\right)\left(\Psi_{s}-\frac{\pi \sqrt{-1}}{4} \tilde{\delta}_{D_{o}}+\frac{\pi \sqrt{-1}}{4 n}\left(* \delta_{D_{o}}\right) \wedge \omega_{\Gamma \backslash \mathfrak{D}}\right) .
\end{aligned}
$$


Remark 7.6.1. From Corollary 3.2.1 we readily have the equation

$$
\triangle G_{s}=-\left(\left(c_{\mathfrak{g}} s\right)^{2}-n^{2}\right) G_{s}-2 \pi\left(* \delta_{D_{o}}\right) .
$$

Indeed, the Laplacian for the function is given by Proposition 7.2.1 (3) in terms of the Casimir element. We also note that the measure of $\mathfrak{D}$ used to define the current $G_{s}$ is differently normalized from Corollary 3.2.1 (see Lemma 7.2.2).

\section{$\S 7.7 . \quad$ Proof of Theorem 7.6.1}

The proof of Theorem 7.6.1 is given at the last part of this subsection after proving several lemmas. Throughout this subsection, $s$ denotes a complex number with $\operatorname{Re}(s)>\rho_{0}=c_{\mathfrak{g}}^{-1} n$.

For a smooth compactly supported function $\varphi$ on $\Gamma \backslash G_{\mathbf{R}}$, we put

$$
[\varphi](g)=\int_{\Gamma_{H} \backslash H_{\mathbf{R}}} \varphi(h g) d \dot{h}, \quad g \in G_{\mathbf{R}} .
$$

For simplicity we also write $[\varphi](t)$ in place of $[\varphi]\left(\exp \left(t Y_{0}\right)\right)$.

Lemma 7.7.1. Let $\varphi \in C_{\mathrm{c}}^{\infty}\left(\Gamma \backslash G_{\mathbf{R}}\right)$. Then for any $\epsilon>0$, we have the estimate

$$
|[\varphi](t)| \prec e^{t \epsilon}, \quad\left|\frac{d}{d t}[\varphi](t)\right| \prec e^{t \epsilon}, \quad t \geqslant 0 .
$$

Proof. With the same notation as in Theorem 3.2.1, since $\varphi$ is compactly supported we have the estimate

$$
|(\varphi * X)(\kappa g)| \prec a_{G}(g)^{\epsilon \rho_{G}}, \quad \kappa \in C, g \in \mathcal{S}
$$

for any $X \in U\left(\mathfrak{g}_{\mathbf{C}}\right)$ and $\epsilon>0$. Hence similarly as in the proof of Theorem 3.2.1, we have the desired estimate of $[\varphi]$.

For a given $\varphi \in A_{c}^{1,1}(\Gamma \backslash \mathfrak{D})$ with $\tilde{\varphi}=\sum_{i, j=0}^{n-1} \varphi_{i j} \cdot \omega_{i} \wedge \bar{\omega}_{j}$, we put

$$
\begin{aligned}
& {[\varphi]_{\tau_{0}}=\left[\left\langle v_{0}, \tilde{\varphi}\right\rangle\right]=\sum_{i=0}^{n-1}\left[\varphi_{i i}\right],} \\
& {[\varphi]_{\tau_{11}}=\left[\left\langle v_{11}, \tilde{\varphi}\right\rangle\right]=\sum_{i=1}^{n-1}\left[\varphi_{i i}\right]-(n-1)\left[\varphi_{00}\right] .}
\end{aligned}
$$

Lemma 7.7.2. Let $\varphi \in A_{c}^{1,1}(\Gamma \backslash \mathfrak{D})$. Then we have

$$
\begin{aligned}
\left\langle\tilde{\delta}_{D_{o}}, * \varphi\right\rangle & =-2 \sqrt{-1}\left[\varphi_{00}\right](0), \\
\left\langle\left(* \delta_{D_{o}}\right) \wedge \omega_{\Gamma \backslash \mathfrak{D}}, * \varphi\right\rangle & =-2 \sqrt{-1}[\varphi]_{\tau_{0}}(0) .
\end{aligned}
$$


Proof. Put

$$
\tilde{\varphi}(g)=\sum_{i, j=0}^{n-1} \varphi_{i j}(g)\left(\omega_{i} \wedge \bar{\omega}_{j}\right), \quad g \in G_{\mathbf{R}}
$$

with $\varphi_{i j} \in C_{\mathrm{c}}^{\infty}\left(\Gamma \backslash G_{\mathbf{R}}\right)$. We first prove (7.7.2). Since $(* \varphi)^{\sim}(g)$ equals

$$
\left(\frac{\sqrt{-1}}{2}\right)^{n-2}\left(\sum_{i=0}^{n-1} \varphi_{i i}(g) \cdot \prod_{k \neq i} \omega_{k} \wedge \bar{\omega}_{k}-\sum_{i, j=0, i \neq j}^{n-1} \varphi_{i j}(g) \cdot \omega_{i} \wedge \bar{\omega}_{j} \prod_{k \neq i, j} \omega_{k} \wedge \bar{\omega}_{k}\right)
$$

by the formula in Proposition 7.2.1 (1), we have

$$
\left(\omega_{\Gamma \backslash \mathfrak{D}} \wedge * \varphi\right)^{\sim}(g)=\tilde{\omega}_{\mathfrak{D}}(g) \wedge * \tilde{\varphi}(g)=-2 \sqrt{-1}\left(\sum_{i=0}^{n-1} \varphi_{i i}(g)\right) \cdot \tilde{v}_{\Gamma \backslash \mathfrak{D}} .
$$

Hence $\left\langle\left(* \delta_{D_{o}}\right) \wedge \omega_{\Gamma \backslash \mathfrak{D}}, * \varphi\right\rangle$, which is $\left\langle\left(* \delta_{D_{o}}\right), \omega_{\Gamma \backslash \mathfrak{D}} \wedge * \varphi\right\rangle$ by definition, equals

$$
\int_{\Gamma_{H} \backslash \mathfrak{D}_{H}} j^{*}\left(*\left(\omega_{\Gamma \backslash \mathfrak{D}} \wedge * \varphi\right)\right) v_{\Gamma_{H} \backslash \mathfrak{D}_{H}}=-2 \sqrt{-1} \int_{\Gamma_{H} \backslash H_{\mathbf{R}}} \sum_{i=0}^{n-1} \varphi_{i i}(h) d \dot{h} .
$$

Thus we have (7.7.2). To prove (7.7.1), we have to rewrite the pullback $j^{*}$ on forms via the identification (7.2.1). Let $\operatorname{pr}_{H}: \bigwedge^{p} \mathfrak{p}_{+}^{*} \wedge \wedge^{q} \mathfrak{p}_{-}^{*} \rightarrow \bigwedge^{p}(\mathfrak{p} \cap \mathfrak{h})_{+}^{*} \wedge$ $\bigwedge^{q}(\mathfrak{p} \cap \mathfrak{h})_{-}^{*}$ be the natural map induced by the canonical inclusion $(\mathfrak{p} \cap \mathfrak{h})_{ \pm} \hookrightarrow$ $\mathfrak{p}_{ \pm}$. We have $\operatorname{pr}_{H}\left(\omega_{i_{1}} \wedge \cdots \wedge \omega_{i_{p}} \wedge \bar{\omega}_{j_{1}} \wedge \cdots \wedge \bar{\omega}_{j_{q}}\right)=0$ if 0 occurs in the sequence $i_{\nu}, j_{\mu}$. Then the pullback $j^{*}: A^{p, q}(\Gamma \backslash \mathfrak{D}) \rightarrow A^{p, q}\left(\Gamma_{H} \backslash \mathfrak{D}_{H}\right)$ corresponds to the map

$$
C^{\infty}\left(G_{\mathbf{R}} ; \sigma_{p q}^{*}\right)^{\Gamma} \rightarrow C^{\infty}\left(H_{\mathbf{R}} ; \sigma_{p q}^{H^{*}}\right)^{\Gamma_{H}}, \quad f \mapsto \operatorname{pr}_{H}\left(f \mid H_{\mathbf{R}}\right) .
$$

Here $\sigma_{p q}^{H}$ denotes the counterpart for $H$ of $\sigma_{p q}$. Hence, using (7.7.3) we have

$$
\left(j^{*}(* \varphi)\right)^{\sim}(h)=-2 \sqrt{-1} \varphi_{00}(h) \cdot \tilde{v}_{\Gamma_{H} \backslash \mathfrak{D}_{H}}, \quad h \in H_{\mathbf{R}} .
$$

Thus $\left\langle\tilde{\delta}_{D_{o}}, * \varphi\right\rangle$ equals

$$
\int_{\Gamma_{H} \backslash \mathfrak{D}_{H}} j^{*}(* \varphi)=-2 \sqrt{-1} \int_{\Gamma_{H} \backslash H_{\mathbf{R}}} \varphi_{00}(h) d \dot{h}=-2 \sqrt{-1}\left[\varphi_{00}\right](0) .
$$

This proves (7.7.1).

Lemma 7.7.3. Let $\varphi \in A_{\mathrm{c}}^{1,1}(\Gamma \backslash \mathfrak{D})$. Then we have

$$
\begin{aligned}
\left\langle G_{s} \wedge \omega_{\Gamma \backslash \mathfrak{D}}, * \varphi\right\rangle & =-\frac{4 \pi \sqrt{-1}}{c_{\mathfrak{g}}^{2}} \int_{0}^{\infty} \gamma_{\mathfrak{h} \backslash \mathfrak{g}}(t) \phi_{s}^{(2)}\left(a_{t}\right) \cdot[\varphi]_{\tau_{0}}(t) d t \\
\left\langle\Psi_{s}, * \varphi\right\rangle & =-\frac{2 \pi}{c_{\mathfrak{g}}^{2}} \int_{0}^{\infty} \gamma_{\mathfrak{h} \backslash \mathfrak{g}}(t) f_{s}(t) \cdot[\varphi]_{\tau_{11}}(t) d t .
\end{aligned}
$$


Proof. We only give a proof of the second formula, because the first one is proved in a similar way. Using the integration formula (1.3.2) with noting the normalization of Haar measures (see Lemma 7.2.2), we have that $\left\langle\Psi_{s}, * \varphi\right\rangle$ equals

$$
\begin{aligned}
\int_{\Gamma \backslash \mathcal{D}} \Psi_{s} \wedge(* \varphi) & =\int_{\Gamma \backslash G_{\mathbf{R}}} *\left(\tilde{\Psi}_{s}(g) \wedge * \tilde{\varphi}(g)\right) d v(\dot{g}) \\
& =\int_{\Gamma \backslash G_{\mathbf{R}}} *\left(\sum_{\gamma \in \Gamma_{H} \backslash \Gamma} \psi_{s}(\gamma g) \wedge * \tilde{\varphi}(g)\right) d v(\dot{g}) \\
& =\int_{\Gamma_{H} \backslash G_{\mathbf{R}}} *\left(\psi_{s}(g) \wedge * \tilde{\varphi}(g)\right) d v(\dot{g}) \\
& =2 \pi c_{\mathfrak{g}}^{-2} \int_{0}^{\infty} \gamma_{\mathfrak{h} \backslash \mathfrak{g}}(t) f_{s}(t) *\left(\check{v}_{11} \wedge[* \tilde{\varphi}](t)\right) d t
\end{aligned}
$$

where $\check{v}_{11}$ is the $M$-invariant tensor in $\mathfrak{p}_{+}^{*} \wedge \mathfrak{p}_{-}^{*}$ which corresponds to $v_{11}$ by the identification $\mathfrak{p}_{-} \wedge \mathfrak{p}_{+} \cong \mathfrak{p}_{+}^{*} \wedge \mathfrak{p}_{-}^{*}$. By the formula (7.7.3) of $(* \varphi)^{\sim}$, we have

$$
\check{v}_{11} \wedge\left[(* \varphi)^{\sim}\right](t)=-[\varphi]_{\tau_{11}}(t) \cdot \tilde{v}_{\mathfrak{D}}
$$

after a computation. By substituting the right-hand side of this equality for $\check{v}_{11} \wedge\left[(* \varphi)^{\sim}\right](t)$ in the last formula in (7.7.6), we have the desired formula.

Lemma 7.7.4. Let $\varphi \in A_{\mathrm{c}}^{1,1}(\Gamma \backslash \mathfrak{D})$.

(1) We have

$$
\begin{aligned}
\frac{1}{4}\left[\left(\bar{\partial}^{*} \partial^{*} \varphi\right)^{\sim}\right](t)= & \left(\frac{c_{\mathfrak{g}}}{2}\right)^{2} \mathcal{L}\left[\varphi_{00}\right](t)-\frac{c_{\mathfrak{g}}}{2}\left(\tanh (t) \frac{d}{d t}\right. \\
& \left.+2 \rho_{0} \tanh ^{2}(t)+\frac{2}{\cosh ^{2}(t)}\right)[\varphi]_{\tau_{11}}(t)
\end{aligned}
$$

(2) We have

$$
\begin{aligned}
(7.7 .8)-\frac{1}{4}\left\langle\partial \bar{\partial} G_{s}, * \varphi\right\rangle= & \frac{\pi}{2} \int_{0}^{\infty} \gamma_{\mathfrak{h} \backslash \mathfrak{g}}(t) \phi_{s}^{(2)}\left(a_{t}\right) \cdot \mathcal{L}\left[\varphi_{00}\right](t) d t \\
& +\frac{\pi}{c_{\mathfrak{g}}} \int_{0}^{\infty} \gamma_{\mathfrak{h} \backslash \mathfrak{g}}(t) \tanh (t) \frac{d}{d t} \phi_{s}^{(2)}\left(a_{t}\right) \cdot[\varphi]_{\tau_{11}}(t) d t .
\end{aligned}
$$

Proof. (1) We show the formula when $(\mathfrak{g}, \mathfrak{h})$ is of type $(\mathrm{O})$. Noting that the two operations $R$ and [ ] are commutative, from the first formula 
in Proposition 7.2.1 (2), we have

$$
\frac{1}{4}\left[\left(\bar{\partial}^{*} \partial^{*} \varphi\right)^{\sim}\right](t)=\sum_{i, j=0}^{n-1}\left\langle X_{i} \wedge \bar{X}_{j}, R_{X_{j} \bar{X}_{i}}[\tilde{\varphi}]\left(a_{t}\right)\right\rangle .
$$

Substituting the expressions given in Lemma 7.1.2 for $R_{X_{j} \bar{X}_{i}}[\tilde{\varphi}]$ in the righthand side of the equality above, and then using the formula

$$
\begin{gathered}
\left\langle X^{\prime} \wedge X^{\prime \prime}, \sigma_{11}^{*}(Z) \eta\right\rangle=-\left\langle\sigma_{11}(Z)\left(X^{\prime} \wedge X^{\prime \prime}\right), \eta\right\rangle, \\
X^{\prime} \in \mathfrak{p}_{+}, X^{\prime \prime} \in \mathfrak{p}_{-}, Z \in \mathfrak{k}_{\mathbf{C}}, \eta \in \mathfrak{p}_{+}^{*} \wedge \mathfrak{p}_{-}^{*},
\end{gathered}
$$

we have

$$
\begin{aligned}
& \frac{1}{4}\left[\left(\bar{\partial}^{*} \partial^{*} \varphi\right)^{\sim}\right](t)=\sum_{i=1}^{n-1}\left\langle X_{i} \wedge \bar{X}_{i},\left(\tanh (t) \frac{d}{d t}+\frac{\sqrt{-1}}{2} \sigma_{11}^{*}\left(Z_{0}\right)\right)[\tilde{\varphi}](t)\right\rangle \\
& +\sum_{i, j=1}^{n-1}\left\langle\sigma_{11}\left(Z_{i} Z_{j}\right)\left(X_{i} \wedge \bar{X}_{j}\right), \tanh ^{2}(t)[\tilde{\varphi}](t)\right\rangle \\
& +\sum_{i=1}^{n-1}\left(\left\langle-\sigma_{11}\left(Z_{i}\right)\left(X_{i} \wedge \bar{X}_{0}\right), \tanh (t) \frac{d}{d t}[\tilde{\varphi}](t)\right.\right. \\
& \left.+\frac{1}{\cosh ^{2}(t)}[\tilde{\varphi}](t)\right\rangle+\left\langle\frac{\sqrt{-1}}{2} \sigma_{11}\left(Z_{i} Z_{0}\right)\left(X_{i} \wedge \bar{X}_{0}\right),[\tilde{\varphi}](t)\right\rangle \\
& \left.-\left\langle\sigma_{11}\left(Z_{i}\right)\left(X_{i} \wedge \bar{X}_{0}\right),[\tilde{\varphi}](t)\right\rangle\right) \\
& +\sum_{j=1}^{n-1}\left(\left\langle-\sigma_{11}\left(Z_{j}\right)\left(X_{0} \wedge \bar{X}_{j}, \tanh (t) \frac{d}{d t}[\tilde{\varphi}](t)\right\rangle\right.\right. \\
& +\left\langle\frac{\sqrt{-1}}{2} \sigma_{11}\left(Z_{0} Z_{j}\right)\left(X_{0} \wedge \bar{X}_{j}\right),[\tilde{\varphi}](t)\right\rangle \\
& \left.+\left\langle\sigma_{11}\left(Z_{j}\right)\left(X_{0} \wedge \bar{X}_{j}\right), \tanh ^{2}(t)[\tilde{\varphi}](t)\right\rangle\right) \\
& +\left\langle X_{0} \wedge \bar{X}_{0},\left(\frac{d^{2}}{d t^{2}}+\operatorname{coth}(t) \frac{d}{d t}\right)[\tilde{\varphi}](t)\right\rangle \\
& -\frac{\sqrt{-1}}{2}\left\langle\sigma_{11}\left(Z_{0}\right)\left(X_{0} \wedge \bar{X}_{0}\right),[\varphi](t)\right\rangle \\
& +\frac{1}{4 \tanh ^{2}(t)}\left\langle\sigma_{11}\left(Z_{0}^{2}\right)\left(X_{0} \wedge \bar{X}_{0}\right),[\tilde{\varphi}](t)\right\rangle .
\end{aligned}
$$


By using (7.1.2) and (7.1.10), we have

$$
\begin{aligned}
\sigma_{11}\left(Z_{i} Z_{j}\right)\left(X_{i} \wedge \bar{X}_{j}\right) & =\left(\delta_{i j}+1\right)\left(-X_{i} \wedge \bar{X}_{i}+X_{0} \wedge \bar{X}_{0}\right) \\
\sigma_{11}\left(Z_{i}\right)\left(X_{i} \wedge \bar{X}_{0}\right) & =\sigma_{11}\left(Z_{i}\right)\left(X_{0} \wedge \bar{X}_{i}\right)=-X_{0} \wedge \bar{X}_{0}+X_{i} \wedge \bar{X}_{i}, \\
\sigma_{11}\left(Z_{0}\right)\left(X_{i} \wedge \bar{X}_{j}\right) & =\sigma_{11}\left(Z_{0}\right)\left(X_{i} \wedge \bar{X}_{0}\right)=0
\end{aligned}
$$

for $1 \leqslant i, j \leqslant n-1$. A straightforward computation with the aid of these formulas, the right-hand side of (7.7.10) turns out to be equal to that of (7.7.7). The unitary case is treated similarly.

(2) Since $\bar{\partial} \partial * \varphi=*\left(\bar{\partial}^{*} \partial^{*} \varphi\right)$ for $\varphi \in A_{\mathrm{c}}^{1,1}(\Gamma \backslash \mathfrak{D})$, the coupling $-\left\langle\partial \bar{\partial} G_{s}, * \varphi\right\rangle$, that is $\left\langle G_{s}, \bar{\partial} \partial * \varphi\right\rangle$ by definition, equals

$$
\begin{aligned}
\left\langle G_{s}, *\left(\bar{\partial}^{*} \partial^{*} \varphi\right)\right\rangle & =\int_{\Gamma \backslash \mathcal{D}} G_{s} \wedge\left(* \bar{\partial}^{*} \partial^{*} \varphi\right) \\
& =\int_{\Gamma \backslash G_{\mathbf{R}}} G_{s}(g)\left(\bar{\partial}^{*} \partial^{*} \varphi\right)^{\sim}(g) d v(\dot{g}) \\
& =\int_{\Gamma \backslash G_{\mathbf{R}}}\left(\sum_{\gamma \in \Gamma_{H} \backslash \Gamma} \phi_{s}^{(2)}(\gamma g)\right)\left(\bar{\partial}^{*} \partial^{*} \varphi\right)^{\sim}(g) d v(\dot{g}) \\
& =\int_{\Gamma_{H} \backslash G_{\mathbf{R}}} \phi_{s}^{(2)}(g)\left(\bar{\partial}^{*} \partial^{*} \varphi\right)^{\sim}(g) d v(\dot{g}) \\
& =2 \pi c_{\mathfrak{g}}^{-2} \int_{0}^{\infty} \gamma_{\mathfrak{h} \backslash \mathfrak{g}}(t) \phi_{s}^{(2)}\left(a_{t}\right)\left[\left(\bar{\partial}^{*} \partial^{*} \varphi\right)^{\sim}\right](t) d t .
\end{aligned}
$$

Substituting the expression in the right hand side of $(7.7 .7)$ for $\left[\left(\bar{\partial}^{*} \partial^{*} \varphi\right)^{\sim}\right](t)$ in the last integrand, and then using integration by parts, we have

$$
\begin{aligned}
- & \frac{1}{4 \pi}\left\langle\partial \bar{\partial} G_{s}, * \varphi\right\rangle \\
= & \frac{1}{2} \int_{0}^{\infty} \gamma_{\mathfrak{h} \backslash \mathfrak{g}}(t) \phi_{s}^{(2)}\left(a_{t}\right) \cdot \mathcal{L}\left[\varphi_{00}\right](t) d t \\
& -\frac{1}{c_{\mathfrak{g}}} \int_{0}^{\infty} \gamma_{\mathfrak{h} \backslash \mathfrak{g}}(t) \phi_{s}^{(2)}\left(a_{t}\right) \cdot\left(\tanh (t) \frac{d}{d t}+2 \rho_{0} \tanh ^{2}(t)+\frac{2}{\cosh ^{2}(t)}\right)[\varphi]_{\tau_{11}}(t) d t \\
= & \frac{1}{2} \int_{0}^{\infty} \gamma_{\mathfrak{h} \backslash \mathfrak{g}}(t) \phi_{s}^{(2)}\left(a_{t}\right) \cdot \mathcal{L}\left[\varphi_{00}\right](t) d t \\
& -\frac{1}{c_{\mathfrak{g}}}\left[\gamma_{\mathfrak{h} \backslash \mathfrak{g}}(t) \phi_{s}^{(2)}\left(a_{t}\right) \tanh (t)[\varphi]_{\tau_{11}}(t)\right]_{0}^{\infty} \\
& +\frac{1}{c_{\mathfrak{g}}} \int_{0}^{\infty} \gamma_{\mathfrak{h} \backslash \mathfrak{g}}(t)[\varphi]_{\tau_{11}}(t) \\
& \times\left(\gamma_{\mathfrak{h} \backslash \mathfrak{g}}(t)^{-1} \frac{d}{d t} \tanh (t) \gamma_{\mathfrak{h} \backslash \mathfrak{g}}(t)-2 \rho_{0} \tanh ^{2}(t)-\frac{2}{\cosh ^{2}(t)}\right) \phi_{s}^{(2)}\left(a_{t}\right) d t .
\end{aligned}
$$


Since $\gamma_{\mathfrak{h} \backslash \mathfrak{g}}(t)=\sinh (t)(\cosh (t))^{2 \rho_{0}-1}$, we have

$$
\gamma_{\mathfrak{h} \backslash \mathfrak{g}}(t)^{-1} \frac{d}{d t} \tanh (t) \gamma_{\mathfrak{h} \backslash \mathfrak{g}}(t)-2 \rho_{0} \tanh ^{2}(t)-\frac{2}{\cosh ^{2}(t)}=\tanh (t) \frac{d}{d t}
$$

by a simple computation. Moreover using the estimates of $\phi_{s}^{(2)}\left(a_{t}\right)$ derived from Corollary 2.4.1 and those of $[\varphi]_{\tau_{11}}(t)$ in Lemma 7.6.1, we can show

$$
\left[\gamma_{\mathfrak{h} \backslash \mathfrak{g}}(t) \phi_{s}^{(2)}\left(a_{t}\right) \tanh (t)[\varphi]_{\tau_{11}}(t)\right]_{0}^{\infty}=0 .
$$

Summing up, we have the desired formula.

Lemma 7.7.5. Let $\varphi \in A_{\mathrm{c}}^{1,1}(\Gamma \backslash \mathfrak{D})$.

(1) We have

$$
\frac{1}{2}[\Delta \varphi]_{\tau_{11}}(t)=-\frac{c_{\mathfrak{g}}^{2}}{2}\left(\mathcal{L}+\frac{4 \rho_{0}}{\cosh ^{2}(t)}\right)[\varphi]_{\tau_{11}}(t)
$$

(2) We have

$$
\frac{1}{2}\left\langle\Delta \Psi_{s}, * \varphi\right\rangle=\pi \int_{0}^{\infty} \gamma_{\mathfrak{h} \backslash \mathfrak{g}}(t) f_{s}(t) \cdot\left(\mathcal{L}+\frac{4 \rho_{0}}{\cosh ^{2}(t)}\right)[\varphi]_{\tau_{11}}(t) d t .
$$

Proof. (1) We prove the formula for the orthogonal case, because that for the unitary case is similarly proved. By the second formula in Proposition 7.2.1 $(2), 2^{-1}\left\langle v_{11},(\triangle \varphi)^{\sim}(g)\right\rangle$ equals

$$
\begin{aligned}
& -\sum_{k=0}^{n-1}\left(R_{X_{k}} R_{\bar{X}_{k}}+R_{\bar{X}_{k}} R_{X_{k}}\right)\left\langle v_{11}, \tilde{\varphi}(g)\right\rangle \\
& \quad-\sum_{k=0}^{n-1} \sum_{i=1}^{n-1}\left(R_{\left[X_{i}, \bar{X}_{k}\right]}\left\langle X_{k} \wedge \bar{X}_{i}, \tilde{\varphi}(g)\right\rangle+R_{\left[\bar{X}_{i}, X_{k}\right]}\left\langle X_{i} \wedge \bar{X}_{k}, \tilde{\varphi}(g)\right\rangle\right) \\
& \quad+(n-1) \sum_{k=1}^{n-1}\left(R_{\left[X_{0}, \bar{X}_{k}\right]}\left\langle X_{k} \wedge \bar{X}_{0}, \tilde{\varphi}(g)\right\rangle+R_{\left[\bar{X}_{0}, X_{k}\right]}\left\langle X_{0} \wedge \bar{X}_{k}, \tilde{\varphi}(g)\right\rangle\right) \\
& =-\sum_{k=0}^{n-1}\left(2 R_{X_{k} \bar{X}_{k}}+R_{\left[\bar{X}_{k}, X_{k}\right]}\right)\left\langle v_{11}, \tilde{\varphi}(g)\right\rangle \\
& \quad-\sum_{k=1}^{n-1} \sum_{i=1}^{n-1}\left(R_{\left[X_{i}, \bar{X}_{k}\right]}\left\langle X_{k} \wedge \bar{X}_{i}, \tilde{\varphi}(g)\right\rangle+R_{\left[\bar{X}_{i}, X_{k}\right]}\left\langle X_{i} \wedge \bar{X}_{k}, \tilde{\varphi}(g)\right\rangle\right)
\end{aligned}
$$




$$
\begin{aligned}
& -\sum_{i=1}^{n-1}\left(R_{\left[X_{i}, \bar{X}_{0}\right]}\left\langle X_{0} \wedge \bar{X}_{i}, \tilde{\varphi}(g)\right\rangle+R_{\left[\bar{X}_{i}, X_{0}\right]}\left\langle X_{i} \wedge \bar{X}_{0}, \tilde{\varphi}(g)\right\rangle\right) \\
& +(n-1) \sum_{k=1}^{n-1}\left(R_{\left[X_{0}, \bar{X}_{k}\right]}\left\langle X_{k} \wedge \bar{X}_{0}, \tilde{\varphi}(g)\right\rangle+R_{\left[\bar{X}_{0}, X_{k}\right]}\left\langle X_{0} \wedge \bar{X}_{k}, \tilde{\varphi}(g)\right\rangle\right) \\
& +(n-1)\left(R_{\left[X_{0}, \bar{X}_{0}\right]}+R_{\left[\bar{X}_{0}, X_{0}\right]}\right)\left\langle X_{0} \wedge \bar{X}_{0}, \varphi(g)\right\rangle .
\end{aligned}
$$

The second term and the fifth one in the right-hand side of the equality above vanish, because of the relation $[X, Y]=-[Y, X]$; the third term cancels a part of the fourth one by the same relation. Hence we have

$$
\begin{aligned}
& \frac{1}{2}\left\langle v_{11},(\triangle \varphi)^{\sim}(g)\right\rangle \\
& =-\sum_{k=0}^{n-1}\left(2 R_{X_{k} \bar{X}_{k}}+R_{\left[\bar{X}_{k}, X_{k}\right]}\right)\left\langle v_{11}, \tilde{\varphi}(g)\right\rangle \\
& \quad+n \sum_{k=1}^{n-1}\left(R_{\left[X_{0}, \bar{X}_{k}\right]}\left\langle X_{k} \wedge \bar{X}_{0}, \tilde{\varphi}(g)\right\rangle+R_{\left[\bar{X}_{0}, X_{k}\right]}\left\langle X_{0} \wedge \bar{X}_{k}, \tilde{\varphi}(g)\right\rangle\right) .
\end{aligned}
$$

By a computation with the aid of the formulas in Lemma 7.1.1 (c) and the formula (7.1.10), we can prove

$$
\left[\bar{X}_{i}, X_{i}\right]=\sqrt{-1} Z_{0}, \quad\left[\bar{X}_{j}, X_{0}\right]=Z_{j}, \quad\left[X_{j}, \bar{X}_{0}\right]=Z_{j}
$$

for $0 \leqslant i, j \leqslant n-1$. Using these relations and the formula (7.7.9), we can rewrite the right-hand side of (7.7.14) as

$$
\begin{aligned}
& -\sum_{k=0}^{n-1}\left(2\left\langle v_{11}, R_{X_{k} \bar{X}_{k}} \tilde{\varphi}(g)\right\rangle-\sqrt{-1}\left\langle\sigma_{11}\left(Z_{0}\right) v_{11}, \tilde{\varphi}(g)\right\rangle\right) \\
& \quad+n \sum_{k=1}^{n-1}\left(\left\langle\sigma_{11}\left(Z_{k}\right)\left(X_{k} \wedge \bar{X}_{0}\right), \tilde{\varphi}(g)\right\rangle+\left\langle\sigma_{11}\left(Z_{k}\right)\left(X_{0} \wedge \bar{X}_{k}\right), \tilde{\varphi}(g)\right\rangle\right) .
\end{aligned}
$$

The remaining part of the proof is quite similar to that of Lemma 7.7.4: first, putting $g=a_{t}$, we substitute the expressions of $R_{X_{k} \bar{X}_{k}} \tilde{\varphi}$ in Lemma 7.1.2 for $R_{X_{k} \bar{X}_{k}} \tilde{\varphi}$ in the formula above, and then move the operators $\sigma_{11}^{*}(Z)$ with $Z \in \mathfrak{k}$ from the right position to the left position in the brackets $\langle$,$\rangle by (7.7.9), and$ finally use the relations

$$
\begin{aligned}
\sigma_{11}\left(Z_{0}\right) v_{11} & =0 \\
\sigma_{11}\left(Z_{i}^{2}\right) v_{11} & =2 n\left(X_{0} \wedge \bar{X}_{0}-X_{i} \wedge \bar{X}_{i}\right), \\
\sigma_{11}\left(Z_{i}\right)\left(X_{i} \wedge \bar{X}_{0}\right) & =\sigma_{11}\left(Z_{i}\right)\left(X_{0} \wedge \bar{X}_{i}\right)=X_{i} \wedge \bar{X}_{i}-X_{0} \wedge \bar{X}_{0}
\end{aligned}
$$


with $1 \leqslant i \leqslant n-1$, that are obtained from (7.7.11). After a computation, we consequently arrive at the expression

$$
-2\left(\mathcal{L}+\frac{2 n}{\cosh ^{2}(t)}\right)\left\langle v_{11}, \tilde{\varphi}\left(a_{t}\right)\right\rangle
$$

to conclude the proof of (1). We prove (2). Similarly as in the proof of Lemma 7.7.3, we have

$$
\frac{1}{2}\left\langle\triangle \Psi_{s}, * \varphi\right\rangle=-2 \pi c_{\mathfrak{g}}^{-2} \int_{0}^{\infty} \gamma_{\mathfrak{h} \backslash \mathfrak{g}}(t) f_{s}(t) \cdot[\triangle \varphi]_{\tau_{11}}(t) d t .
$$

Then by (1), we have the conclusion.

Lemma 7.7.6. Let $\beta: \mathbf{R} \rightarrow \mathbf{C}$ be $a C^{\infty}$-function such that

$$
|\beta(t)| \prec e^{t \epsilon}, \quad\left|\beta^{\prime}(t)\right| \prec e^{t \epsilon}, \quad t \geqslant 0
$$

for any $\epsilon>0$.

(1) We have

$$
\int_{0}^{\infty} \gamma_{\mathfrak{h} \backslash \mathfrak{g}}(t) \phi_{s}^{(2)}\left(a_{t}\right) \cdot\left(\mathcal{L}+\rho_{0}^{2}-s^{2}\right) \beta(t) d t=\beta(0) .
$$

(2) We have

$$
\int_{0}^{\infty} \gamma_{\mathfrak{h} \backslash \mathfrak{g}}(t) f_{s}(t) \cdot\left(\mathcal{L}+\frac{4 \rho_{0}}{\cosh ^{2}(t)}+\rho_{0}^{2}-s^{2}\right) \beta(t) d t=-\frac{c_{\mathfrak{g}}\left(s^{2}-\rho_{0}^{2}\right)}{4 \rho_{0}} \beta(0) .
$$

Proof. (1) This is proved in the course of the proof of Theorem 3.2.1.

(2) The proof is similar to (1). Firstly by integration by parts we have

$$
\begin{aligned}
& \int_{0}^{\infty} \gamma_{\mathfrak{h} \backslash \mathfrak{g}}(t) f_{s}(t) \cdot\left(\mathcal{L}+\frac{4 \rho_{0}}{\cosh ^{2}(t)}+\rho_{0}^{2}-s^{2}\right) \beta(t) d t \\
& =\lim _{\eta \rightarrow+\infty}\left(\gamma_{\mathfrak{h} \backslash \mathfrak{g}}(\eta) f_{s}(\eta) \beta^{\prime}(\eta)-\gamma_{\mathfrak{h} \backslash \mathfrak{g}}(\eta) f_{s}^{\prime}(\eta) \beta(\eta)\right) \\
& +\lim _{\epsilon \rightarrow+0}\left(-\gamma_{\mathfrak{h} \backslash \mathfrak{g}}(\epsilon) f_{s}(\epsilon) \beta^{\prime}(\epsilon)+\gamma_{\mathfrak{h} \backslash \mathfrak{g}}(\epsilon) \beta(\epsilon) f_{s}^{\prime}(\epsilon)\right) \\
& \quad+\int_{0}^{\infty} \gamma_{\mathfrak{h} \backslash \mathfrak{g}}(t) \beta(t)\left(\mathcal{L}+\frac{4 \rho_{0}}{\cosh ^{2}(t)}+\rho_{0}^{2}-s^{2}\right) f_{s}(t) d t .
\end{aligned}
$$


The integrand of the third term in the right hand side of (7.7.15) is zero by Corollary 7.5.1 (2). By Corollary 7.5.2 (1) and the estimate of $\beta$ and $\beta^{\prime}$, we have

$$
\begin{aligned}
& \lim _{\eta \rightarrow+\infty}\left(\gamma_{\mathfrak{h} \backslash \mathfrak{g}}(\eta) f_{s}(\eta) \beta^{\prime}(\eta)-\gamma_{\mathfrak{h} \backslash \mathfrak{g}}(\eta) f_{s}^{\prime}(\eta) \beta(\eta)\right)=0, \\
& \lim _{\epsilon \rightarrow+0} \gamma_{\mathfrak{h} \backslash \mathfrak{g}}(\epsilon) f_{s}(\epsilon) \beta^{\prime}(\epsilon)=0 .
\end{aligned}
$$

By Corollary 7.5.2 (2), noting $\gamma_{\mathfrak{h} \backslash \mathfrak{g}}(t)=t+O\left(t^{2}\right)$ around $t=0$, we have

$$
\lim _{\epsilon \rightarrow+0} \gamma_{\mathfrak{h} \backslash \mathfrak{g}}(\epsilon) f_{s}^{\prime}(\epsilon) \beta(\epsilon)=-\frac{c_{\mathfrak{g}}\left(s^{2}-\rho_{0}^{2}\right)}{4 \rho_{0}} \beta(0) .
$$

Summing up we have the conclusion.

Now let us begin the proof of Theorem 7.6.1.

$<$ Proof of $(7.6 .1)>$

Applying Lemma 7.7.6 (1) to the first integral in the right-hand side of the formula (7.7.8), we get

$$
\begin{aligned}
-\frac{1}{4 \pi}\left\langle\partial \bar{\partial} G_{s}, * \varphi\right\rangle= & \frac{1}{2}\left(\left(s^{2}-\rho_{0}^{2}\right) \int_{0}^{\infty} \gamma_{\mathfrak{h} \backslash \mathfrak{g}}(t) \phi_{s}^{(2)}\left(a_{t}\right) \cdot\left[\varphi_{00}\right](t) d t+\left[\varphi_{00}\right](0)\right) \\
& +\frac{1}{c_{\mathfrak{g}}} \int_{0}^{\infty} \gamma_{\mathfrak{h} \backslash \mathfrak{g}}(t) \tanh (t) \frac{d}{d t} \phi_{s}^{(2)}\left(a_{t}\right) \cdot[\varphi]_{\tau_{11}}(t) d t .
\end{aligned}
$$

Adding (7.7.8) to $\left(8 \pi \rho_{0} \sqrt{-1}\right)^{-1} c_{\mathfrak{g}}\left(s^{2}-\rho_{0}^{2}\right)$-times the formula obtained from (7.7.4) by replacing $[\varphi]_{\tau_{0}}$ for $[\varphi]_{\tau_{11}}+n\left[\varphi_{00}\right]$, we have

$$
\begin{aligned}
- & \frac{1}{4 \pi}\left\langle\partial \bar{\partial}^{*} G_{s}, * \varphi\right\rangle+\frac{c_{\mathfrak{g}}\left(s^{2}-\rho_{0}^{2}\right)}{8 \sqrt{-1} \rho_{0} \pi}\left\langle G_{s} \wedge \omega_{\Gamma \backslash \mathfrak{D}}, * \varphi\right\rangle \\
= & \frac{1}{2}\left(\left(s^{2}-\rho_{0}^{2}\right) \int_{0}^{\infty} \gamma_{\mathfrak{h} \backslash \mathfrak{g}}(t) \phi_{s}^{(2)}\left(a_{t}\right) \cdot\left[\varphi_{00}\right](t) d t+\left[\varphi_{00}\right](0)\right) \\
& +\frac{1}{c_{\mathfrak{g}}} \int_{0}^{\infty} \gamma_{\mathfrak{h} \backslash \mathfrak{g}}(t)\left(\tanh (t) \frac{d}{d t}-\frac{s^{2}-\rho_{0}^{2}}{2 \rho_{0}}\right) \phi_{s}^{(2)}\left(a_{t}\right) \cdot[\varphi]_{\tau_{11}}(t) d t \\
& -\frac{\left(s^{2}-\rho_{0}^{2}\right) n}{2 \rho_{0} c_{\mathfrak{g}}} \int_{0}^{\infty} \gamma_{\mathfrak{h} \backslash \mathfrak{g}}(t) \phi_{s}^{(2)}\left(a_{t}\right) \cdot\left[\varphi_{00}\right](t) d t \\
= & \frac{s^{2}-\rho_{0}^{2}}{2}\left(1-\frac{n}{\rho_{0} c_{\mathfrak{g}}}\right) \int_{0}^{\infty} \gamma_{\mathfrak{h} \backslash \mathfrak{g}}(t) \phi_{s}^{(2)}\left(a_{t}\right) \cdot\left[\varphi_{00}\right](t) d t+\frac{1}{2}\left[\varphi_{00}\right](0) \\
& +\frac{2}{c_{\mathfrak{g}}^{2}} \int_{0}^{\infty} \gamma_{\mathfrak{h} \backslash \mathfrak{g}}(t) f_{s}(t) \cdot[\varphi]_{\tau_{11}}(t) d t .
\end{aligned}
$$


Note that to have the last equality, we use Corollary 7.5.1. Now since $n=c_{\mathfrak{g}} \rho_{0}$, the first term in the right-hand side of the last equality is zero. Hence from (7.7.16), (7.7.1) and (7.7.5), we finally have

$$
\begin{gathered}
-\frac{1}{4 \pi}\left\langle\partial \bar{\partial} G_{s}, * \varphi\right\rangle+\frac{c_{\mathfrak{g}}\left(s^{2}-\rho_{0}^{2}\right)}{8 \sqrt{-1} \rho_{0} \pi}\left\langle G_{s} \wedge \omega_{\Gamma \backslash \mathfrak{D}}, * \varphi\right\rangle \\
=\frac{\sqrt{-1}}{4}\left\langle\tilde{\delta}_{D_{o}}, * \varphi\right\rangle-\frac{1}{\pi}\left\langle\Psi_{s}, * \varphi\right\rangle
\end{gathered}
$$

for any $\varphi \in A_{\mathrm{c}}^{1,1}(\Gamma \backslash \mathfrak{D})$. Since $*$ gives an isomorphism $A_{\mathrm{c}}^{1,1}(\Gamma \backslash \mathfrak{D}) \cong$ $A_{\mathrm{c}}^{n-1, n-1}(\Gamma \backslash \mathfrak{D})$, we have done.

$<$ Proof of $(7.6 .2)>$

From (7.7.13), using Lemma 7.7.6 (2), we have

$$
\begin{aligned}
& \frac{2}{\pi}\left\langle\Delta \Psi_{s}, * \varphi\right\rangle \\
& =-\frac{c_{\mathfrak{g}}\left(s^{2}-\rho_{0}^{2}\right)}{\rho_{0}}\left([\varphi]_{\tau_{0}}(0)-n\left[\varphi_{00}\right](0)\right)-4\left(\rho_{0}^{2}-s^{2}\right) \\
& \quad \times \int_{0}^{\infty} \gamma_{\mathfrak{h} \backslash \mathfrak{g}}(t) f_{s}(t) \cdot[\varphi]_{\tau_{11}}(t) d t .
\end{aligned}
$$

From (7.7.17), (7.7.5), (7.7.2) and (7.7.1), we obtain

$$
\begin{aligned}
\frac{2}{\pi}\left\langle\Delta \Psi_{s}, * \varphi\right\rangle & \\
= & \frac{c_{\mathfrak{g}}\left(s^{2}-\rho_{0}^{2}\right)}{\rho_{0}}\left(-\frac{\sqrt{-1}}{2}\left\langle\left(* \delta_{D_{o}}\right) \wedge \omega_{\Gamma \backslash \mathfrak{D}}, * \varphi\right\rangle\right. \\
& \left.+\frac{\sqrt{-1} n}{2}\left\langle\tilde{\delta}_{D_{o}}, * \varphi\right\rangle-\frac{2 c_{\mathfrak{g}} \rho_{0}}{\pi}\left\langle\Psi_{s}, * \varphi\right\rangle\right) .
\end{aligned}
$$

Noting $c_{\mathfrak{g}} \rho_{0}=n$ and $* A_{\mathrm{c}}^{1,1}(\Gamma \backslash \mathfrak{D})=A_{\mathrm{c}}^{n-1, n-1}(\Gamma \backslash \mathfrak{D})$, we have the desired formula.

\section{$\S 7.8 . \quad$ Unitary case}

Let $G$ and $H$ be as in Section 6 and $\Gamma$ a neat arithmetic subgroup of $G_{\mathbf{Q}}$. Then by Theorem 6.3 .1 , the $(0,0)$-current $G_{s}$ on $\Gamma \backslash \mathfrak{D}$, which is at first defined on $\operatorname{Re}(s)>n$ can be continued meromorphically to whole $\mathbf{C}$. It has a simple pole at $s=n$ and the residue of $G_{s}$ at $s=n$ is a constant function on $\Gamma \backslash \mathfrak{D}$. As a corollary of this property of $G_{s}$ and the differential equations (7.6.1) and (7.6.2), we have 


\section{Theorem 7.8.1.}

(1) The (1,1)-current valued function $s \mapsto \Psi_{s}$, which is at first defined on $\operatorname{Re}(s)>n$, can be continued meromorphically to whole $\mathbf{C}$. It is holomorphic at the point $s=n$.

(2) The value $\Psi_{n}$ of $\Psi_{s}$ at $s=n$ is represented by a $C^{\infty}$-form in $A^{1,1}(\Gamma \backslash \mathfrak{D})$. Moreover it is harmonic, i.e.,

$$
\triangle \Psi_{n}=0
$$

(3) Let $\mathcal{G}$ be the normalized Green function defined by

$$
\mathcal{G}=\lim _{s \rightarrow n}\left(G_{s}-\frac{\kappa}{s-n}\right)
$$

with $\kappa=(2 n \operatorname{vol}(\Gamma \backslash \mathfrak{D}))^{-1} \operatorname{vol}\left(\Gamma_{H} \backslash \mathfrak{D}_{H}\right)$. Then we have

$$
\sqrt{-1} \partial \bar{\partial} \mathcal{G}-\pi \tilde{\delta}_{D_{o}}=\kappa \cdot \omega_{\Gamma \backslash \mathfrak{D}}+4 \sqrt{-1} \Psi_{n}
$$

Proof. Since $G_{s}$ is meromorphic on $\mathbf{C}$, the meromorphic continuation of $\Psi_{s}$ is obtained by the differential equation (7.6.1). The residue $\operatorname{Res}_{s=n} G_{s}=\kappa$ is a constant function. Hence $\operatorname{Res}_{s=n}\left(\partial \bar{\partial} G_{s}\right)=\partial \bar{\partial} \kappa=0$. Thus in the left-hand side of (7.6.1), the first term produces no pole at $s=n$; the second term is also holomorphic because the factor $s-n$ cancels the singularity of $G_{s}$ at $s=n$. Therefore (7.6.1) means $\Psi_{s}$ is holomorphic at $s=n$, hence (1) follows. By comparing the constant term at $s=n$ of both sides of the equality (7.6.1), we obtain (7.8.2). Let $s \rightarrow n$ in (7.6.2). Then noting $\Psi_{s}$ is holomorphic at $s=n$, the right-hand side of (7.6.2) goes to zero. Hence we have (7.8.1). Since $\triangle$ is an elliptic differential operator, (7.8.1) implies the first statement of (2). This completes the proof.

Remark 7.8.1. When $(\mathfrak{g}, \mathfrak{h})$ is of type $(\mathrm{U})$ or $(\mathrm{O})$ and $\Gamma \backslash \mathfrak{D}$ is compact, one obtains the meromorphic continuation of the function $G_{s}$ on $s$ to the whole $\mathbf{C}$ by the same method as in Section 6 . It is much simpler because we do not need to consider the contribution from the continuous spectrum. Therefore $G_{s}$ has a simple pole at $s=\rho_{0}=c_{\mathfrak{g}}^{-1} n$ and $\kappa=\operatorname{Res}_{s=c_{g}^{-1} n} G_{s}$ is a constant function. Let $\mathcal{G}$ be the constant term of $G_{s}$ at $s=c_{\mathfrak{g}}^{-1} n$. By the same way as above, we have that $\Psi_{s}$ is holomorphic at $s=c_{\mathfrak{g}}^{-1} n$ and

$$
\sqrt{-1} \partial \bar{\partial} \mathcal{G}-\pi \tilde{\delta}_{D_{o}}=\kappa \cdot \omega_{\Gamma \backslash \mathfrak{D}}+4 \sqrt{-1} \Psi_{c_{\mathfrak{g}}^{-1} n}
$$


which is the Chern form of the divisor $D_{o}([8,1.3])$. Moreover each term in the right-hand side of the equality corresponds to the contribution of the cohomological representations (the trivial representation and $\pi^{1,1}$ ) to the second cohomology group $H^{2}(\Gamma \backslash \mathfrak{D} ; \mathbf{C})$. (For $\pi^{1,1}$ see Remark 7.5.1.)

The function (or $(0,0)$-current) $G_{s}(z)$ has a simple pole at $s=n$ on one hand. On the other hand, the Eisenstein series $E^{i}(s)$ also have simple poles at $s=n$; their residues are proportional to the inverse of the volume $\operatorname{vol}(\Gamma \backslash \mathfrak{D})$. Hence if we make a linear combination

$$
\tilde{E}(s: z)=\sum_{i=1}^{r} c_{i} \cdot E^{i}(s: z), \quad z \in \Gamma \backslash \mathfrak{D}
$$

of Eisenstein series suitably so that

$$
\operatorname{Res}_{s=n} G_{s}(z)=\operatorname{Res}_{s=n} \tilde{E}(s: z),
$$

then the limit

$$
\hat{\mathcal{G}}(z)=\lim _{s \rightarrow n}\left(G_{s}(z)-\sum_{i=1}^{r} c_{i} \cdot E^{i}(s: z)\right)
$$

makes sense. In this way, we have a renormalized Green function $\hat{\mathcal{G}}$. But to determine the optimal choice of the constants $c_{i}$ 's is another problem.

\section{References}

[1] Borel, A., Introduction aux groupes arithmétique, Hermann, Paris, 1969.

[2] Borel, A. and Wallach, N., Continuous cohomology, discrete subgroups, and representations of reductive groups, Ann. of Math. Stud. 94, Princeton University Press, Princeton, 1980.

[3] Borcherds, R. E., Automorphic forms with singularities on Grassmannians, Invent. Math., 132 (1998), 491-562.

[4] Bruinier, J., Borcherds products and Chern classes of Hirzebruch-Zagier divisors, Invent. Math., 138 (1999), 51-83.

[5] B Borcherds products on $O(2, l)$ and Chern classes of Heegner divisors, Preprint (http://www.math.uni-mannheim.de/ fga/index.htm;Preprint No.2, 2000).

[6] Elstrodt, J., Die Resolvente zem Eigenwertproblem der automorphen Formen in der hyperbolischen Ebene, Teil I, Math. Ann., 203 (1973), 295-330.

[7] Flensted-Jensen, M., Spherical functions on a real semisimple Lie group. A method of reduction to the complex case, J. Funct. Anal., 30 (1978), 106-146.

[8] Gillet, H. and Soulé, C., Arithmetic intersection theory, Publ. of I.H.E.S, 72 (1990), 94-174.

[9] Gon, Y., Generalized Whittaker functions on $S U(2,2)$ with respect to the Siegel parabolic subgroup, Mem. Amer. Math. Soc., 155 no. 738, (2002).

[10] Gross, B. H. and Zagier, D. B., Heegner points and derivatives of $L$-series, Invent. Math., 84 (1986), 225-320. 
[11] Harish-Chandra, Automorphic forms on semisimple Lie groups, Lecture Note in Math., 62 Springer-Verlag, Berlin, 1968.

[12] Heckman, G. and Schlichtkrull, H., Harmonic analysis and special functions on symmetric spaces, Perspect. Math. 16, Academic Press, 1994.

[13] Hejahl, D. A., The Selberg trace formula for PSL(2, R) II, Lecture Notes in Math., 1001, Springer Verlag, Berlin, 1983.

[14] Hörmander, L., Linear Partial differential operators, Springer Verlag, Berlin, 1963.

[15] Ishii, T., Siegel-Whittaker functions on $S_{0}(2, q)$ for class one principal series respresentations, doctorial dissertation, University of Tokyo, 2002.

[16] Ishikawa, Y., The generalized Whittaker functions for $S U(2,1)$ and the Fourier expansion of automorphic forms, J. Math. Sci. Univ. Tokyo, 6 no.3, (1999), 477-526.

[17] Kobayashi, T., Singular unitary representations and discrete series for indefinite Stiefel manifolds $U(p, q ; \mathbf{F}) / U(p-m, q ; \mathbf{F})$, Mem. Amer. Math. Soc., 95, no.462 (1992).

[18] Knapp, A. W., Representation theory of semisimple groups, Princeton University Press, 1986.

[19] Miatello, R. and Wallach, N., Automorphic forms constructed from Whittaker vectors, J. Funct. Anal., 86 (1989), 411-487.

[20] $\quad$ The resolvent of the Laplacian on locally symmetric spaces, J. Differential Geom., 36 (1992), 663-698.

[21] Kudla, S. S., Theta-functions and Hilbert modular forms, Nagoya Math. J., 69 (1978), 97-106.

[22] _ Central derivatives of Eisenstein series and hight pairings, Ann. of Math., 146 no.3, (1997), 545-646.

[23] Langlands, R. P., On the functional equations satisfied by Eisenstein series, Lecture Notes in Math., 544 Springer-Verlag, Berlin, 1976.

[24] Lebedev, N. N., Special functions and their applications, Dover Publications, INC., New York, 1972.

[25] Oda, T., On modular forms associated with indefinite quadratic forms of signature (2,n-2), Math. Ann., 231 (1977), 97-144.

[26] Osborn, M. S. and Warner, G., The Selberg trace formula, I: $\Gamma$-rank one lattices, $J$. Reine Angew. Math., 324 (1981), 1-113.

[27] Rallis, S. and Schiffmann, G., Automorphic cusp forms constructed from the Weil representation, Bull. Amer. Math. Soc., 83 no. 2, (1977), 267-270.

[28] Roelcke, W., Das Eigenwertproblem der automorphen Formen in der hyperbolishen Ebene I, II., Math. Ann., 167 (1966), 292-337, ibid., 168 (1967), 261-324.

[29] Rossmann, W., The structure of semisimple symmetric spaces, Can. J. Math., XXXI no. 1, (1979), 157-180.

[30] Schmid, W., On realization of the discrete series of a semisimple Lie group, Rice University Studies, 56 (1970), 99-108.

[31] Tong, Y. L. and Wang, S. P., Geometric realization of discrete series for semisimple symmetric spaces, Invent. Math., 96 no.2, (1989), 425-458.

[32] Vigneras, M. F., Série théta associée a des formes quadratiques indéfinies, Modular functions of one variable, VI, (Proc. Second Internat. Conf., Univ. Bonn, Bonn, 1976), 227-239, Lecture Notes in Math., 627 Springer, Berlin, 1977.

[33] Wang, S. P., Embeddings of Flensted-Jensen modules in $L^{2}(\Gamma \backslash G)$ in the non-compact case, Lecture Notes in Math., 1447 (1989), pp. 333-356.

[34] Whittaker, E. T. and Watson, G. N., A course of modern analysis, Cambridge University Press, 1963.

[35] Weil, A., Introduction a la théorie des variétés Kähleriennes, Hermann, Paris 1958.

[36] Wallach, N. R., On the constant term of a square integrable automorphic form, in Operator Algebras and Group representations II, Monogr. Stud. Math., 17, pp. 227-237 Pitman, London, 1984. 